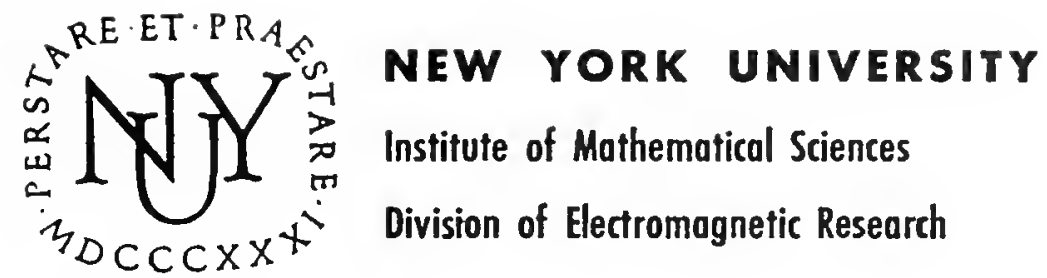

RESEARCH REPORT NO. EM-81

\title{
Asymptotic Solution of Some Diffraction Problems
}

J. B. KELLER, R. M. LEWIS and B. D. SECKLER

CONTRACT NO. AF $19(122)-42$

JUNE 1955 

NEW YORK UNIVERSITY

Institute of Mathematical Sciences

Division of Electromagnetic Research

Research Report No. EM-81

\title{
ASYMPTOTIC SOLUTION OF SOME DIFFRACTION PROBLEMS
}

\author{
Joseph B. Keller, Robert M. Lewis and Bernard D. Seckler
}

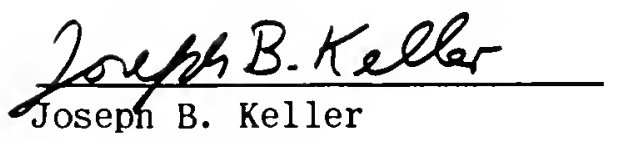

Refat M. Louno

Robert M. Lewis

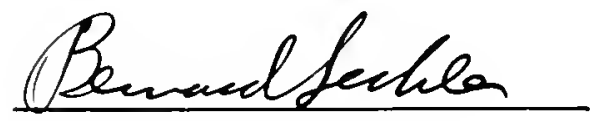

Bernard D. Seckler

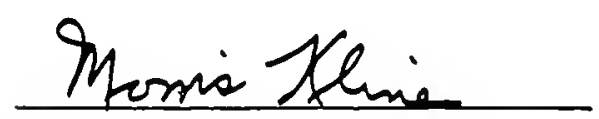

Morris Kline

Project Director

The research reported in this document has been made possible through support and sponsorship extended by the Air Force Cambridge Research Center, under Contract No. AF 19(122)-42. It is published for technical information only and does not necessarily represent recommendations or conclusions of the sponsoring agency. 

Abstract

Various diffraction problems are solved asymptotically in $k=\frac{2 \pi}{\lambda}$ ( $\lambda$ = wavelength) for $k$ large (i.e., $\lambda$ smail). These problems include diffraction of a plane wave by a parabolic cylinder, a paraboloid of revolution, a cylinder and a sphere; diffraction of a spherical wave by a paraboloid of revolution, a hyperboloid of revolution, and a plane interface; diffraction of a cylindrical wave by a parabolic cylinder, a hyperbolic cylinder and a plane interface, etc. The boundary conditions considered are the vanishing of the function, of its normal derivative and the impedance boundary condition. Formulas are obtained for reflection of any wave from any two dimensional surface, and certain formulas are deduced for three dimensional problems. The method employed is that devised by $R . K$. Luneburg and extended by M. KIine. 
1. Introduction 1

2. Formulation of the Method 2

3. Cylindrical Waves 3

General Considerations

Example 1. Diffraction of a Plane Wave by a Wedge or Half-Plane 4

Example 2. Hankel Functions 5

Example 3. Diffraction of a Plane Wave by a Parabolic Cylinder $(u=0) 6$

Example 4. Diffraction of a Plane Wave by a Parabolic Cylinder 9 $\frac{\partial u}{\partial \nu}=0$

Example 5. The Field of a Line Source at One Focus of a Hyperbolic 13 Cylinder $(u=0)$

Example 6. The Field of a Line Source at One Focus of a Hyperbolic 18 Cylinder $\left(\frac{\partial u}{\partial v}=0\right)$

Example 7. The Field of a Iine Source over a Plane Interface 19

4. General Cylindrical Waves 29

5. Spherical Waves 31

General Considerations

Example 8. Diffraction of a Plane Wave by a Cone of Arbitrary 32

Cross-Section

Example 9. Spherical Bessel Functions 33

Example 10. Diffraction of a Plane Wave by a Paraboloid of 33 Revolution ( $u=0$ )

Example 11. Diffraction of a Plane Wave by a Paraboloid of Revolution ( $\frac{\partial u}{\partial v}=0$ )

Example 12. The Field of a Foint Source at one Focus of a Hyperboloid of Revolution ( $u=0$ )

Example 13. The Field of a Point Source at one Focus of a Hyperboloid of Revolution ( $\frac{\partial u}{\partial v}=0$ )

Example 14. The Field of a Point Source Over a Plane Interface 
6. General Plane Waves

44

General Considerations

Example 25. A Line Source at the Focus of a Parabolic Cylinder

45 $(u=0)$

Example 16. A Line Source at the Focus of a Parabolic Cylinder $\left(\frac{\partial u}{\partial v}=0\right)$

Example 17. A Point Source at the Focus of a Paraboloid of Revolution ( $u=0$ )

Example 18. A Point Source at the Focus of a Paraboloid of Revolution $\left(\frac{\partial u}{\partial \nu}=0\right)$

7. Arbitrary Waves

8. Two-dimensional waves

General Considerations

Example 19. Diffraction of a Flane Wave by a Circular Cylinder $(u=0)$

Example 20. Diffraction of a Plane Wave by a Circular Cylinder $\left(\frac{\partial u}{\partial v}=0\right)$

9. Axially Symmetric Waves

General Considerations

Example 21. Diffaction of a Plane Wave by a Sphere $(u=0)$

71

Example 22. Diffraction of a Plane Wave by a Sphere $\left(\frac{\partial u}{\partial v}=0\right)$

10. The Impedance Boundary Condition

General Considerations

Fxample 23. Diffraction of a Plane Wave by a Parabolic Cylinder $\left(\frac{\partial u}{\partial v}=i k Z u\right)$

Fxample 24. Diffraction of a Plane Wave by a Circular Cylinder $\left(\frac{\partial u}{\partial v}=1 k 2 u\right)$ 


\section{Introduction}

Several authors $[1]-[15]$ have recently discussed a method by which the asymptotic expansion with respect to frequency may be determined for periodic solutions of electromagnetic, acoustic, and other linear wave problems. However, virtually no applications of the method have been made. In the present paper we explain the method, and apply it to construct the asymptotic solutions of a varlety of diffraction problems. Many of these problems have not been solved previously. In some cases where the problems have been solved previously we verify that the asymptotic expansions of the known solutions coincide with our asymptotic solutions and compare the exact solutions graphically with the first few terms of the asymptotic solutions.

The asymptotic expanston under consideration here is an expansion of a periodic solution with respect to frequency as the frequency tends to infinity. Thus the expansion is useful at high frequencies, or for short wavelengths. In electromagnetic theory it is therefore useful in optics, in radar problems, etc. In fact the leading term in the expansion is just the so-called geometrical-optics solution.

Mathematically the problem is that of finding the asymptotic expansion of the solution of a boundary-value problem for an elliptic equation. The equation and the boundary data involve a parameter $k$ which is proportional to the frequency of the solution and inversely proportional to the wavelength, and the order of the equation is reduced when $k^{-1}$ is replaced by zero (i.e., when $k$ becomes inf inite). The method employed determines directly the asymptotic expansion with respect to $k$ near $k=\infty$, and thus we can avoid the customary detour of first finding an exact solution and then expanding it asymptotically in $k$. Consequently, the present method can be used in cases where the exact solution has not been found, and this is its main value. But even where the exact solution $1 s$ known and can be expanded, the present method usually yields the expansion more easily. It must be noted, however, that for most problems it has not yet been rigorously proved that the expansion yielded by this method is indeed asymptotic to the exact solution, although formally this appears to be the case.

The numerous problems treated in this paper by the above method are selected as examples of the types of problems for which this method is applicable. On the other hand, it is also apparent that unless the problems correspond to the simplest geometries, the calculations become quite lengthy. In fact the problems considered in this paper were selected partly because the related ray systems are simple, and 
therefore the calculations are relatively easy. Further, it is not yet possible, in general, to treat problems involving diffracted rays [6] by this method. In some problems where such rays do occur the present method can yield only incomplete results, as in examples 1 and 8. However in examples 7 and 14 a special method was devised to treat the diffracted rays, and 1 t is to be expected that more such methods will be devised for other problems.

2. Formulation of the Method

We consider a solution u (scalar or vector) of the reduced wave equation

$$
\left(\nabla^{2}+k^{2}\right) u=0
$$

We assume that $u$ has an asymptotic expansion (for $k \rightarrow \infty$ ) of the form

$$
u \sim e^{i k \Psi} \sum_{n=0}^{\infty} \frac{v_{n}(x, y, z)}{(i k)^{n}} .
$$

We further assume that the asymptotic expansions of the derivatives of $u$ are obt.alned by differentiation of the expansion of $u$. Then inserting (2) into (1) and equating to zero the coefficient of each power of $k$, we obtain the equations

$$
(\nabla \Psi)^{2}=1
$$

$$
2 \nabla v_{n} \cdot \nabla \Psi+v_{n} \nabla^{2} \Psi=-\nabla^{2} v_{n-1}, n=0,1, \ldots ; v_{-1}=0
$$

In (4) the coefficient $v_{-I}$ is identically zeros it has been introduced to permit the equation for $v_{0}$ to be written together with those for the other $v_{n}$ *

Equation (3) is the eiconal equation of geometrical optics, and determines the phase function I. Equations (4) are a recursive system which can be used for determining the $\nabla_{n}$ successively. Since $\nabla v_{n} \cdot \nabla \cdot=\frac{d v_{n}}{d s}$, where $s$ denotes arc length along a ray (i.e., a curve orthogonal to the wavefronts $\mathbf{I}=$ constant), equations ( 4 ) are in fact linear or linary differential equations along the rays. The solution of (4), along a ray, is

$$
v_{n}(s)=v_{n}\left(s_{0}\right) e^{-\frac{1}{2} \int_{s_{0}}^{s} \nabla^{2} Y d s}-\frac{1}{2} \int_{s_{0}}^{s} e^{-\frac{1}{2} \int_{\tau}^{s} \nabla^{2} d s^{1} \nabla^{2} v_{n-1}(\tau) d \tau . ~}
$$

Equations (5) can be written in the simpler form (see [1]). 
(6)

$$
v_{n}(s)=\nabla_{n}\left(s_{0}\right)\left[\frac{G(s)}{G\left(s_{0}\right)}\right]^{1 / 2}-\frac{G^{1 / 2}(s)}{2} \int_{s_{0}}^{s} G^{-1 / 2}(\tau) \nabla^{2} v_{n-1}(\tau) d \tau .
$$

In (6), $G(s)$ denotes the Gaussian curvature, or, in two dimensions, the ordinary curvature, of the wavefront $Y=$ constant at the point $s$ on a ray.

For $n=0$ the integral in (6) is absent since $v_{-1}=0$, so $v_{0}$ is given by the first term. This solution shows that $v_{0}$ varies aloni a ray inversely as the square root of the cross-sectional area of a narrow tube of rays, because $G(s)$ is inversely proportional to this area. Therefore $\nabla_{0}^{2}$ multiplied by this area is constant along each ray, which expresses the conservation of energy within such a tube. Equation (6) also shows that if $\nabla$ is a vector, the orientation of $\nabla_{0}$ is constant along each ray.

The preceding results are not completely new, and have been included here primarily for the sake of completeness and convenience. MoKine ${ }^{[2]}$ has obtained analogous results for Maxwell's equations, and the analogues of equations (3) and (4) for more general linear equations. His method, which is more complicated than the present one, is that of R.K. Luneburg [l] who used it to obtain partial results for Maxwell's equations. Other authors have also obtained partial results for speclal equations - usually the analogues of (3) and of the first of equations (4) and (6), i. a of the equations for which $n=0$. F.G. Friedlander $[3], H . B r$ emer $[4]$, E.T. Copson $[5]$ and J. Riblet (unpublished) also treated Maxwell's equations; S.C. Lowell [12] considered waves in shallow water; J.3. Seller [7] treated the equations of acoustics; G.D. Birkhoff ${ }^{[8]}$, L. Brillouin[9], G. Wentzel [21], P.A.M. Dirac [10] and J.3. Keller $[11]$ treated the Schroedinger equation of quantum mechanics. Particular problems have been solved with the aid of these asymptotic expansions by C. Schensted [13], by K.O. Friedrichs and J.3. Keller ${ }^{[14]}$, and by J.B. Keller [7], [11]. More general asymptotic expansions of solutions of equation ( 1 ) have been considered by F.G. Friedlander and J.B. Keller $[15]$.

In the followng sections we will construct the asymptotic expansions of the solutions of several boundary-value problems by using equations (3) and (6). We will also derive certain general formulas which may be of use in other problems.

\section{Cylindrical Waves}

The rays, which are the orthogonal trajectories of the surfaces constant, are straight Iines, as can be shown from (3). Let us first consider the two-dimensional case in which all the rays are the lines $(\theta=$ constant $)$ 'emerging from an 
origin and the wavefronts are the concentric circles ( $\mathrm{r}=$ constant). Then $\Psi= \pm r+$ constant, and we may without loss of generality choose the constant to be zero since equation ( 1 ) is linear and homogeneous. The curvature of the wavefront $\left(r=\right.$ constant) is $G(r)=r^{-1}$. Thus from $(0)$, usins $r$ instead of $s$, we have

$$
v_{0}(r, \theta)=g_{0}(\theta) r^{-1 / 2},\left[g_{0}(\theta)=r_{0}^{1 / 2}(\theta) v_{0}\left[r_{0}(\theta),-\theta\right]\right] .
$$

The factor $g_{0}(\theta)$ is just $r_{0}^{l / 2} v_{0}\left(r_{0}\right)$ evaluated on the ray $(\theta=$ constant $)$ at $r=r_{0}(\theta)$. If we now use (6) and (7) we find by induction that $v_{n}(r, \theta)$ can be written in the form

$$
v_{n}(r, \theta)=\sum_{j=0}^{n} f_{j n}(\theta) r^{-(1 / 2)-j}
$$

Inserting (8) into ( 6 ) yields the following recursive formulas for $f_{j n}(\theta)$, with $\dot{n} \geq 1:$

$$
f_{j n}(\theta)= \pm \frac{1}{2 j}\left[\left(j-\frac{1}{2}\right)^{2} f_{j-1, n-1}+f_{j-1, n-1}^{n}\right], \quad j \neq 0, n \geq 1,
$$

$$
f_{o n}(\theta)=r_{0}^{1 / 2}(\theta) v_{n}\left[r_{0}(\theta), \theta\right]-\sum_{j=1}^{n} r_{0}^{-j} f_{j n}, \quad n \geq 1 .
$$

In (9) and (10) the upper sign is to be used if $I=+r$ and the lower sign if $I=-r$. The function $f_{00}(\theta)$ is just $g_{0}(\theta)$, and $v_{n}\left[r_{0}(\theta), \theta\right]$ is the value of $v_{n}$ at the point $r_{o}(\theta)$ on the ray $(\theta=$ constant). Combining (2) and ( 8 ) we thus have, for a cylindrical wave,

$$
u \sim \frac{e^{ \pm i k r}}{\sqrt{r}} \sum_{n=0}^{\infty} \frac{1}{(i k)^{n}} \sum_{j=0}^{n} f_{j n}(\theta) r^{-j} .
$$

If the $v_{n}\left[\underline{r}_{0}(\theta), \theta\right]$ are given then $(9)$ and $(10)$ yield all the $f_{j n}(\theta)$ successively, and involve only differentiation. Thus if the expansion of $u$ is given on a curve $r=r(\theta)$ then the $v_{n}\left[r_{0}(\theta), \theta\right]$ are given and (Il) yields the expansion of $f$ the curve. This way of obtaining the asymptotic expansion of a cylindrical wave is the main result of this section, and we will now consider several applications of it. Example 1. Diffraction of a Plane Wave by a Wedge or Half-Plane

In the two-dimensional problem of diffraction of a plane wave by a wedge (perfect conductor or dielectric) or a half-plane, the asymptotic expansion of the 
solution consists of several plane waves and of a diffracted cylindrical wave emanating from the edge. The results (7)-(10) can be applied to this cylindrical wave。 We first observe that in the solution of this problem $k$ and $r$ can occur only in the combination $k r$, since the problem contains no characteristic dimension. In order that the expansion also involve only the combination $k r$, we first multiply (11) by $\mathrm{k}^{-1 / 2}$, which we may do because the equation ( 1 ) is Linear and homogeneous. Now since the coefficient of $v_{n}$ is $k^{-(1 / 2)-n}$, and since $v_{n}$ can contain only this same power of $r$, we must have $f_{j n}=0$ if $j \neq n$. Then from (9) using the solution $Y=r$ for the phase in order that the wave be outgoing, and using the notation $D=\frac{d}{d \theta}$, we have

$$
f_{n n}(\theta)=\frac{1}{2^{n} n !} \prod_{j=1}^{n}\left[\left(j-\frac{1}{2}\right)^{2}+D^{2}\right] g_{0}(\theta) \text {. }
$$

Inserting (12) into (11) we obtain

$$
u(r, \theta) \sim \frac{e^{i k r}}{\sqrt{k r}}\left\{1+\sum_{n=1}^{\infty} \frac{1}{(2 i k r)^{n} n !} \prod_{j=1}^{n}\left[\left(j-\frac{1}{2}\right)^{2}+D^{2}\right]\right\} g_{0}(\theta) .
$$

Equation (13) yields an asymptotic expansion which formally satisfies (1) for any choice of $g_{0}(\theta)$. The present method cannot determine $g_{0}(\theta)$ for the wedge or half-plane problems. However in other problems, some of which are treated in this article, the present method can determine $g_{0}(\theta)$. If for the wedge or half-plane we take $g_{0}(\theta)$ from the first term in the asymptotic expansion of the exact solution, which is known if $u=0$ or $\frac{\partial u}{\partial n}=0$ on the wedge, then (13) will yield all the subsequent terms in the expansion.

Example 2. Hankel Functions

A cylindrical wave solution of ( 1 ) is given by the product of a Hankel function $\mathrm{H}_{\mathrm{m}}^{\mathrm{l}}(\mathrm{kr})$ and $\sin \mathrm{m} \theta$ or $\cos \mathrm{m} \theta$. Thus we have

$$
\mathrm{u}=\mathrm{H}_{\mathrm{m}}^{\mathrm{I}}(\mathrm{kr}) \cos \mathrm{m} \theta
$$

Since this solution is outgoing and involves $\mathrm{k}$ and $\mathrm{r}$ only in the combination $\mathrm{kr}$, its expansion is given by (13) with $g_{0}(\theta)=A \cos m \theta$. The constant $A$ must be adjusted so that (13) agrees with $\left(\mathcal{U}_{1}\right)$. Inserting $g_{0}(\theta)=A \cos m \theta$ into (13) and noting that $D^{2} \cos m \theta=-m^{2} \cos m \theta$ we obtain 


$$
H_{m}^{1}(k r) \sim A \frac{e^{i k r}}{\sqrt{k r}}\left\{1+\sum_{n=1}^{\infty} \frac{1}{(2 i k r)^{n} n_{0}^{1}} \prod_{j=1}^{n}\left[\left(j-\frac{1}{2}\right)^{2}-m^{2}\right]\right\} .
$$

This expansion coincides exactly with the known expansion of $H_{m}^{l}$ if

$$
A=\sqrt{\frac{2}{\pi}} \exp \left[-\frac{1 \pi}{2}\left(m+\frac{1}{2}\right)\right] \quad(\text { See }[20] \text { p. 85, eq. } 1)
$$

If the phase function $Y$ is taken as $-r$ instead of $+r$, we obtain from (9)(II) the expansion of the Hankel function of the second kind, which differs from the above merely in that $i$ is replaced by $-i$, (see [20], p. 85, eq. 2). Example 3. Diffraction of a Plane Wave by a Parabolic Cylinder ( $u=0$ ). We consider a plane wave $e^{-i k x}$ incident from the right along the axis of a parabolic cylinder, and on the outside of it (see Fig. 1). The incident rays are parallel to the axis, and the reflected rays are therefore radial lines which pass through the focus if extended backward, as is well-known from the focussing property of parabolas. Therefore the reflected field is a cylindrical wave which, we will assume, can be described by equation (II). We will write the equation of the parabola of focal length $p$ as

$$
r=r_{0}(\theta)=\frac{2 p}{1+\cos \theta} \text {. }
$$

We impose on the parabola the boundary condition

$$
u(r, \theta)=0 \text { on } r=r_{0}(\theta) \text {. }
$$

Inserting the total field, incident plus reflected, into (16a) we have

$$
e^{-i k r_{0}(\theta) \cos \theta}+e^{i k \Psi\left(r_{0}, \theta\right)} \sum_{n=0}^{\infty} \frac{v_{n}\left(r_{0}, \theta\right)}{(1 k)^{n}} \sim 0 .
$$

Now equating coeffictents of various powers of $k$ in (17) we obtain

$$
\begin{aligned}
& I\left(r_{0}, \theta\right)=-r_{0}(\theta) \cos \theta, \\
& \nabla_{0}\left(r_{0}, \theta\right)=-1, \\
& v_{n}\left(r_{0}, \theta\right)=0, \quad n \geq 1 .
\end{aligned}
$$




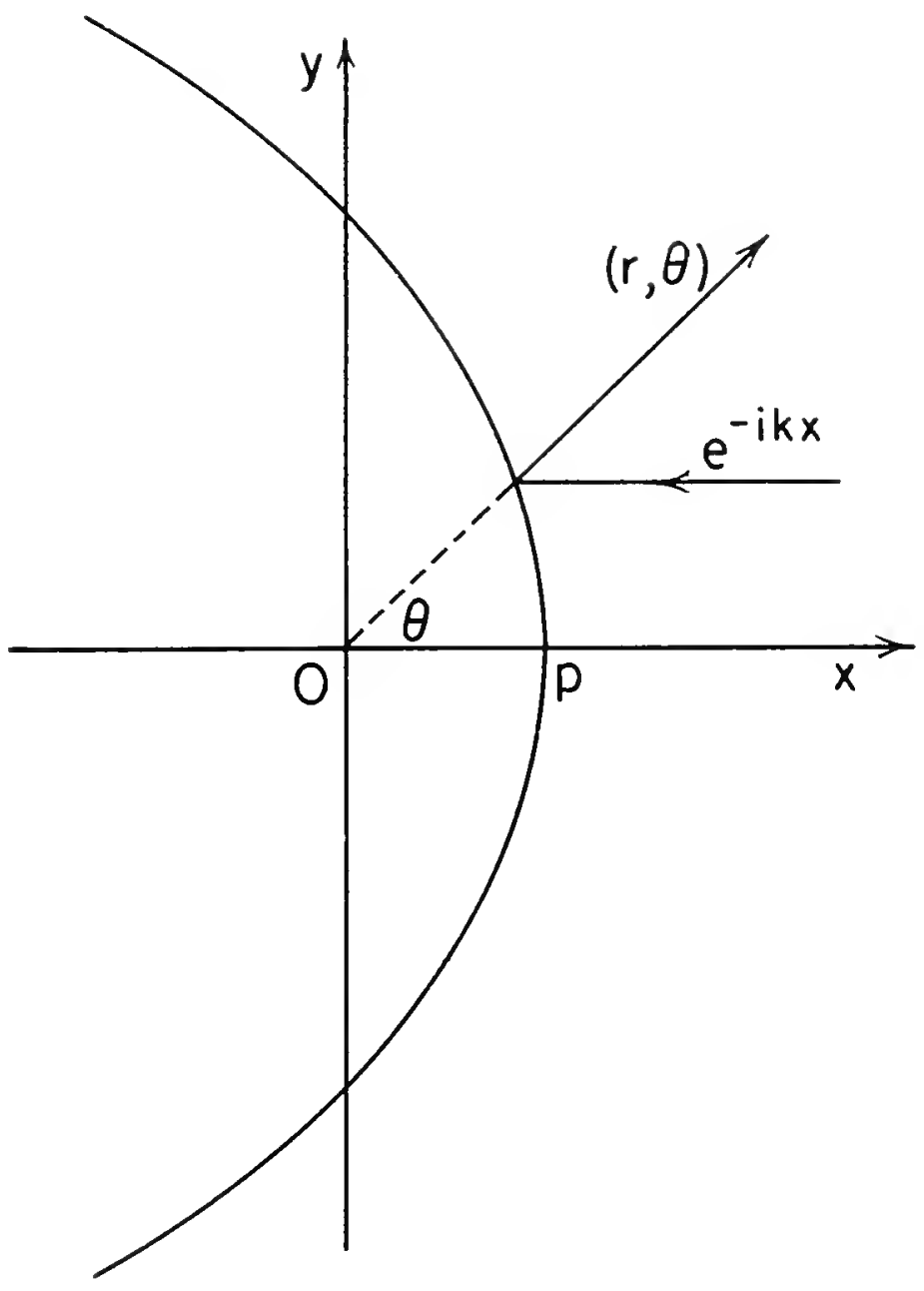

sigure 1

Reflection of a plane wave from a parabolic cylinder, or from a paraboloid of revolution of focal length $p$. The plane wave is incident from the right along the axis of the parabolic cylinder (or paraboloid) and the origin is at the focus. The incident rays are parallel to the axis and the reflected rays pass through the focus if extended backward. 
The phase 1 is determined by (3) and (18), or alternatively by the distances along the rays, and the condition that the wave be outgoing. We have

$$
\Psi(r, \theta)=r-r_{0}(1+\cos \theta)=r-2 p \text {. }
$$

Using (19) in (7) we obtann $g_{0}(\theta) \cdot-r_{0}^{1 / 2}(\theta)$, and thus

$$
\nabla_{0}(r, \theta)=-r_{0}^{1 / 2}(\theta) r^{-1 / 2}=-p^{1 / 2}\left(\sec \frac{\theta}{2}\right) r^{-1 / 2} \text {. }
$$

Now we may determine the $f_{j n}(\theta)$ from (9) and (10) using the boundary conditions (20) and the expression for $g_{0}(\theta)$ given in (22). Upon calculating the first few $f_{j n}$ we find that the $f_{j n}$ have the following form:

$$
f_{j n}(\theta)=a_{j n} p^{(1 / 2)+j-n}\left(\sec \frac{\theta}{2}\right)^{2 j+1}
$$

which can then be proved by induction to hold generally。 In (23) the coeficients $a_{\text {jn }}$ are constants which satisfy the recursion formulas

$$
\begin{array}{ll}
a_{j n}=\frac{1}{2}\left(j-\frac{1}{2}\right) a_{j-1, n-1,} & j \geq 1, n \geq 1 \\
a_{o n}=-\sum_{j=1}^{n} a_{j n^{\prime}} & n \geq 1, \\
a_{O 0}=-1 . &
\end{array}
$$

From $(24)$ and (25) the $a_{\text {fn }}$ can be determined successively, starting with a Given by $(26)$. Collecting our results, we have for the asymptotic expansi on of the solution

$$
u \sim e^{-i k x}+e^{i k(r-2 p)} \sum_{n=0}^{\infty}(i k p)^{-n} \sum_{j=0}^{n} a_{j n}\left(p r^{-1} \sec ^{2} \frac{\theta}{2}\right)^{j+(1 / 2)} .
$$

From equation (27) with the $a_{\text {jn }}$ computed from (24)-(26), we find for the first few terms in the expansion of $u$ 


$$
u \sim e^{-i k x}-\sqrt{\frac{p}{r}} \sec \frac{\theta}{2} e^{i k(r-2 p)}\left\{1-\frac{1}{4 i k p}\left[1-\frac{p}{r} \sec ^{2} \frac{\theta}{2}\right]+\cdots\right\} .
$$

The problem treated in this section was solved exactly by H. Lamb [18] who made use of the separability of the problem in parabolic coordinates. When his solution is expanded asymptotically in $k$, it yields exactly (27). The amplitude and phase of the scattered wave computed from the exact solution are shown in Figures 2 and 3. For comparison the same quantities computed from the first few terms in the asymptotic expansion, i.e., from the terms through $(\mathrm{kp})^{-2}$, are also shown. The agreement is seen to be good when $\mathrm{kp} \geq 2$.

Example 4. Diffraction of a Plane Wave by a Parabolic Cylinder $\left(\frac{\partial u}{\partial v}=0\right)$

We consider the same situation as that of Example 3 but we replace the boundary condition (16a) by the requirement that the normal derivative of $u$ vanish, i.e., that

$$
\frac{\partial u}{\partial \nu}=0 \text { on } r=r_{0}(\theta) \text {. }
$$

Then instead of (17) we obtain

$$
-i k \frac{\partial x}{\partial \nu} e^{-i k x}+e^{i k \Psi}\left(i k \frac{\partial \Psi}{\partial \nu}+\frac{\partial}{\partial \nu}\right) \sum_{n=0}^{\infty} \frac{v_{n}}{(i k)^{n}} \sim 0 \quad \text { on } r=r_{0}(\theta) \text {. }
$$

Equating the coefficients of the first power of $k$ in (30) we find

$$
\begin{array}{ll}
\Psi=-x=-r_{0}(\theta) \cos \theta & \text { on } r=r_{0}(\theta), \\
\frac{\partial \Phi}{\partial \nu}=\frac{\partial x}{\partial \nu} & \text { on } r=r_{0}(\theta) .
\end{array}
$$

From (31), (3) and the outgoing wave condition we find that $\mathbf{Y}$ is given by (21). Then $\frac{\partial \Psi}{\partial \nu}=\frac{\partial x}{\partial \nu}$ on $r=r_{0}(e)$. Therefore (32) yields

$$
v_{0}=1 \text { on } r=r_{0}(E) \text {. }
$$

The coefficients of all higher powers of $k$ yield 


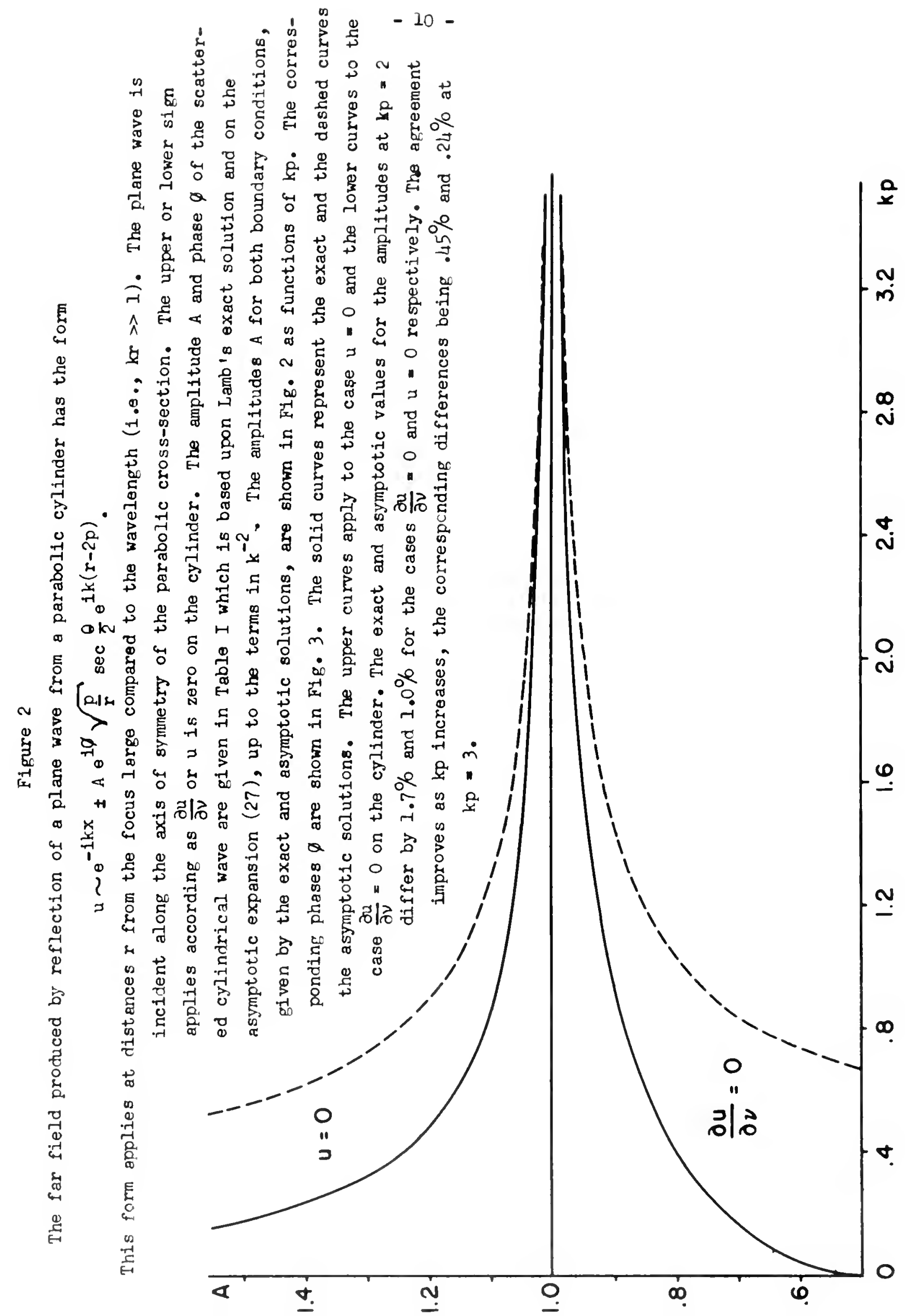




\section{TABLE I}

Scattering From A Parabolic Cylinder

$$
\begin{aligned}
& u=0 \\
& A e^{i \phi}=\left[2 i \sqrt{2 k p} e^{-2 i k p} \int_{\sqrt{2 k p}}^{\infty} e^{i z^{2}} d z\right]^{-1} \\
& A \sim 1+\frac{5}{32(k p)^{2}}+\ldots \cdot \\
& {[E q \cdot(27)]} \\
& \tan \varnothing \sim \frac{1}{4 k p}+\ldots . \\
& A e^{i \phi}=\left[2+2 i \sqrt{2 k p} \int_{\sqrt{2 k p}}^{\infty} e^{i z^{2}} d z\right]^{-1} \\
& A \sim 1-\frac{7}{32(k p)^{2}}+\ldots . \\
& \tan \varnothing \sim-\frac{1}{4 k p}+\ldots .
\end{aligned}
$$

Scattering From A Paraboloid of Revolution

$$
\begin{array}{rl}
u=0 & A e^{i \phi}=\left[-4 i k p e^{-2 i k p} \int_{\sqrt{2 k p}}^{\infty} e^{i z^{2}} \frac{d z}{z}\right]^{-1} \\
A & \sim 1+\frac{3}{8(k p)^{2}}+\ldots \ldots \\
\tan \phi & \sim \frac{1}{2 k p}+\ldots \\
\frac{\partial u}{\partial v}=0 \quad \ldots & \left.e^{i z^{2}} \frac{d z}{z}\right] \\
A e^{i \phi} & =\left[2+4 i k p e^{-2 i k p} \int_{\sqrt{2 k p}}^{\infty}\right. \\
A & \sim 1 \\
\tan \phi & \sim-\frac{5}{8(k p)^{2}}+\ldots . \\
2 k p+\ldots .
\end{array}
$$




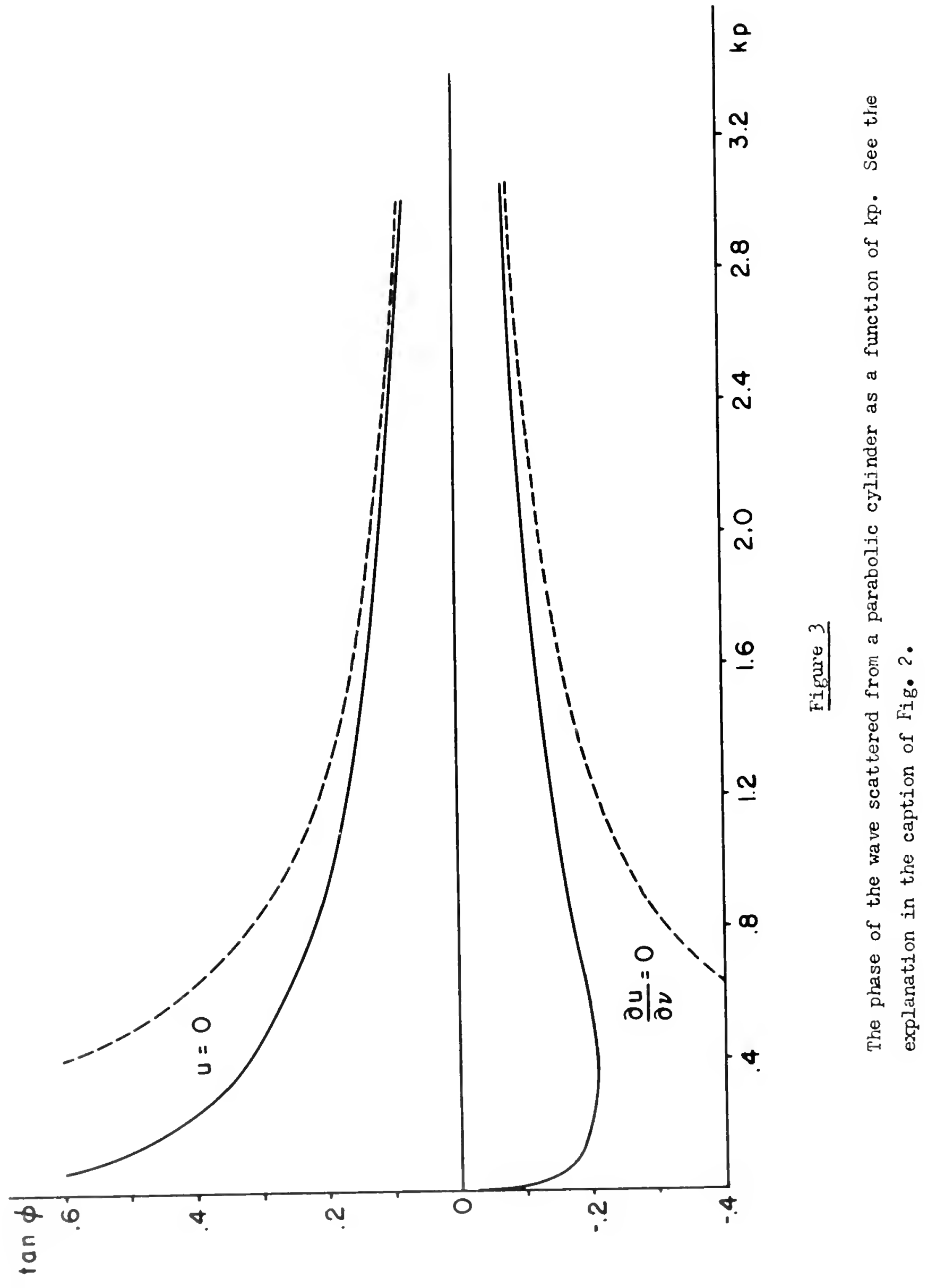




$$
v_{n+1}=-\left(\frac{\partial x}{\partial \nu}\right)^{-1} \frac{\partial v_{n}}{\partial \nu} \quad \text { on } r=r_{0}(\theta) ; \quad n=0,1, \cdots
$$

From $(7)$ and $(33)$ we find $g_{0}(\theta)=r_{0}^{1 / 2}(\theta)$ and

$$
v_{0}(r, \theta)=r_{0}^{1 / 2}(\theta) r^{-1 / 2}
$$

Again from (9) and (10) we find, using the boundary conditions (34) and the expression for $g_{0}(\theta)$ given in (35), that $f_{j n}(\theta)$ has the form (23) and that $a_{j n}$ satisfies the recursion formula (24). However, $a_{00}=+1$ and the $a_{\text {on }}(n>0)$ satisfy (25) with the minus sign in front of the sum replaced by a plus sign. The asymptotic expansion of $\mathfrak{u}$ is agaln given by (27) with the appropriate ${ }_{j n}$, which are different from those of the previous problen due to the changes of sign in (25) and (26).

From (27) and the modified forms of (24) - (26) we find for the first terms in the expansion of $u$

$$
u \sim e^{-i k x}+\sqrt{\frac{p}{r}} \sec \frac{\theta}{2} e^{i k(r-2 p)}\left\{1+\frac{1}{4 i k p}\left[1+\frac{p}{r} \sec ^{2} \frac{\theta}{2}\right]+\cdots\right\} \text {. }
$$

This problem has also been solved exactly by H. Lamb [18]. The asymptotic expansion of his solution is precisely that found here (i.e., (27) with the appropriate $\left.a_{j n}\right)$. The amplitude and phase of his scattered wave are shown in Figures 2 and 3 where they are compared with the corresponding quantities computed frcm (36). The agreement is seen to be gocd when $k p \geq 2$.

Fxample 5. The Field of a Line Source at One Focus of a Byperbolic Cylinder $(u=0)$

Let lis consider the two-dimensional problem of determining the field produced by a line source at one focus of a hyperbolic cylinder on which $u=0$ (see Fig. 4). The field produced by a line source in an unbounded medium is a cylindrical wave which is circularly symmetric, and which is described exactly by 


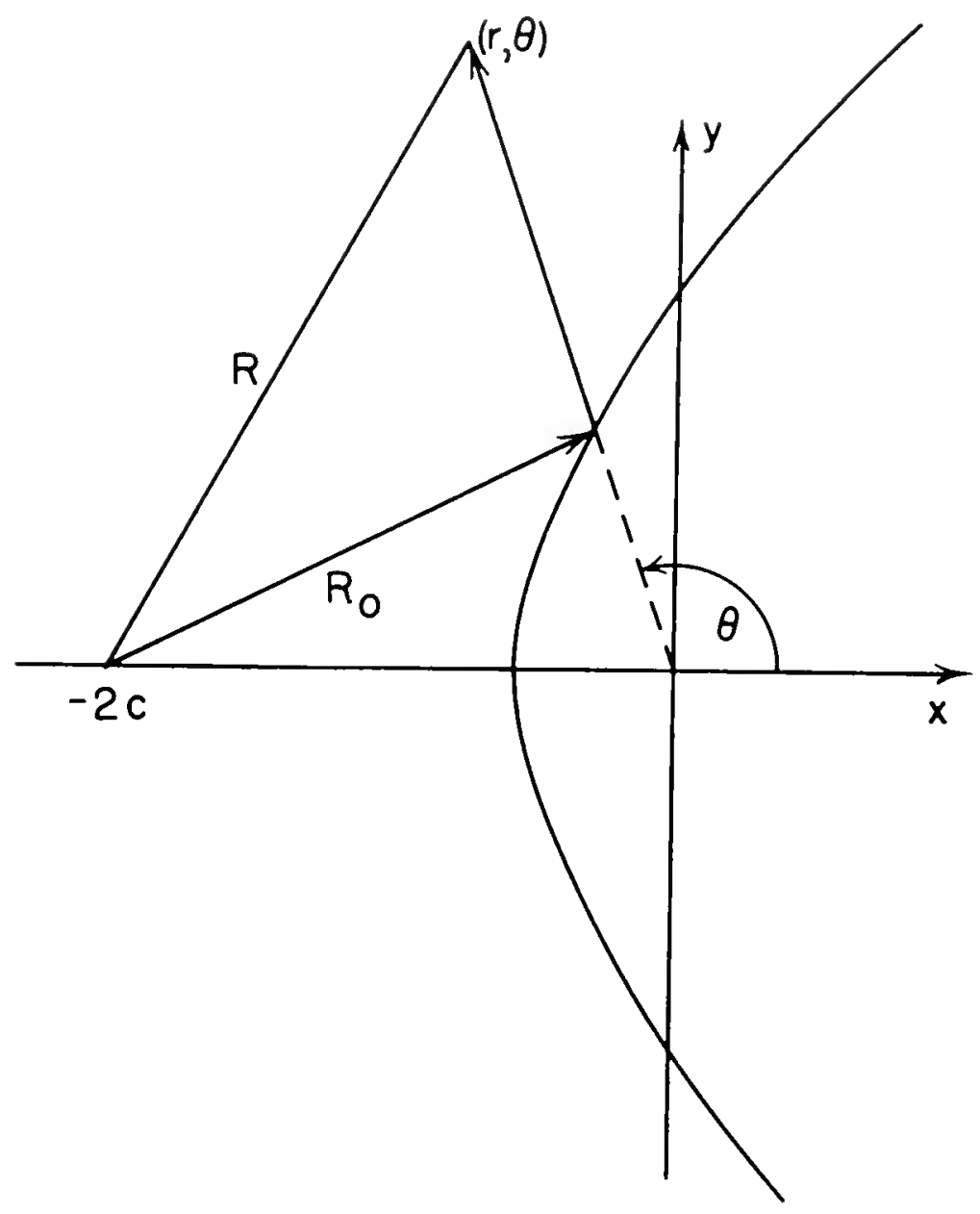

Figure 4

Reflection from a hyperbolic cylinder of a cylindrical wave emanating from a line source at one focal line of the hyperbolic cylinder. This focal line is at $(-2 c, 0)$ while the other focal line is at $(0,0)$. The incj.dent rays are directed radially from the source, and the reflected rays are directed radially from the other focal line (i.e., from the origin in the cross-sectional view). The figure also represents the reflection from a hyperboloid of revolution of a spherical wave emanating from a point source at one focus of the hyperboloid, the origin being at the other fccus。 
equation (14) and asymptotically by equation (15), in both cases with $\mathrm{m}=0$. For the present problem we may take either of these descriptions of the field as the definition of a line source. This cylindrical wave will impinge upon the hyperbolic cylinder and be reflected and diffracted. Due to the focussing property of a hyperbola the reflected rays will be radially directed lines which pass through the other focus of the hyperbola if extended backward. Therefore the wave reflected from the hyperbola is also a cylindrical wave and we may describe it by equation (II). First we write the equation of the hyperbola as

$$
r=(p-q \cos \theta)^{-1}=r_{0}(\theta)
$$

The origin of coordinates is at one focus of the hyperbola and the source is at the other focus, whose cartesian coordinates are $(-2 c, 0)$. The constant $c$ is the focal length of the hyperbola, and the constants $p$ and $q$ are related to the major and minor semi-axes $a$ and $b$ by the equations $p=a b^{-2}, q=c b^{-2}$. Let $R$ denote the distance from the source to any point and let $R_{0}$ denote the distance from the source to a point on the hyperbola. Then $R_{0}=r_{0}+2 a$, by a well-known property of the hyperbola.

Now, using (15) with $m=0$, we may write the expansion of the solution in the form

$$
u \sim e^{i k R} \sum_{n=0}^{\infty} p_{n}(i k R)^{-n-(1 / 2)}+e^{i k \Psi} \sum_{n=0}^{\infty}(i k)^{-n-(1 / 2)} v_{n} \cdot
$$

The constants $\rho_{n}$ are found, from (14), to be given by

$$
\rho_{n}=\sqrt{\frac{2}{\pi}} \frac{[(2 n) !]^{2}}{2^{5 n}(n !)^{3}} \text {. }
$$

The second term in (38) was obtained by multiplying (2) by the constant (ik) $)^{-1 / 2}$, in order that this term, which represents the reflected and diffracted waves, be of the same order as the incident wave. Of course, due to the linearity and homogeneity of (1), this factor does not affect the derivation of equations (7) - (10). 
To determine $I$ and the $v_{n}$ we first use the boundary condition $u=0$ on the hyperbola. From this condition, with u given by (38), we obtain

$$
\begin{aligned}
& I=R_{0}-r_{0}+2 a, \text { on } r=r_{0}(\theta) . \\
& v_{n}=-R_{0}^{-n-(1 / 2)} \rho_{n}, 0 \leq n .
\end{aligned}
$$

By considering distances along the reflected rays, and using the boundary value (40) for on the hyperbola and the outgoing condition for the reflected wave, we find that is piven by

$$
\begin{aligned}
& I=r+2 a \\
& \text { Now we use }(4 I) \text { and }(7) \text { to obtain } v_{0}(r, \theta) \text { : } \\
& v_{0}(r, \theta)=-(w r)^{-I / 2}
\end{aligned}
$$

In (43) we have introduced the new variable which is related to $\theta$ by

$$
\mathrm{W}=\mathrm{P}-\mathrm{Q} \cos \theta
$$$$
P=1+2 a p,
$$$$
Q=2 \mathrm{aq} \text {. }
$$

From (41) and (43) it appears to be convenient to consider the $f_{j n}$ as functions of

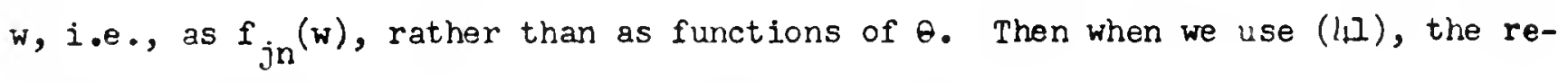
cursion formulas (9) and (10) become the following:

$$
\begin{aligned}
f_{j n}(t)=(2 j)^{-1}\left[\left(j-\frac{1}{2}\right)^{2} f_{j-I, n-1}\right. & +(P-w) f_{j-I, n-1}^{\prime} \\
& +\left(Q^{2}-P^{2}+2 P w-w^{2}\right) f_{j-I, n-1}^{\prime \prime}, j \geq 1, n \geq 1,
\end{aligned}
$$

(46) $f_{o n}(w)=-p_{n}(2 a)^{-n_{w}-1 / 2}\left(1-w^{-1}\right)^{n}-\sum_{j=1}^{n}\left(\frac{w-1}{2 a}\right)^{j} f_{j n}(w), \quad n \geq 1$. Starting with $f_{00}$ which is given by (42) as $f_{00}=-w^{-1 / 2}$, we may compute the $f_{j n}$ successively, using $(45)$ and $(46)$. Upon computing a few, we find a certain regularity in the $f_{j n}$ which leads us to assume that $f_{j n}$ has the form

$$
f_{j n}(w)=\sum_{t=0}^{2 n-j} c_{j t n}(2 a)^{-n+j} w^{*}-[(1 / 2)+j+t] .
$$


The $c_{j t n}$ are constants. The form $(47)$ can be proved to be correct by induction, using (48) and (46), if the $c_{j t n}$ satisfy the recursion formulas

$$
\begin{aligned}
c_{j t n}=(2 j)^{-1} & {\left[\left\{\left(j-\frac{1}{2}\right)^{2}-\left(\frac{1}{2}+j+t\right)^{2}\right\} c_{j-1, t+1, n-1}+2 P\left(j+t-\frac{1}{2}\right)(t+j) c_{j-1, t, n-1}\right.} \\
& \left.+\left(Q^{2}-P^{2}\right)\left(j+t-\frac{3}{2}\right)\left(j+t-\frac{1}{2}\right) c_{j-1, t-1, n-1}\right], 1 \leq j \leq n, 0 \leq t \leq 2 n-j,
\end{aligned}
$$

$$
c_{\text {otn }}=\rho_{n}(-1)^{t+1}\left(\begin{array}{l}
n \\
t
\end{array}\right)-\sum_{j=1}^{n} \sum_{s=0}^{2 n-j} c_{j s n}\left(\begin{array}{c}
j \\
t-s
\end{array}\right)(-1)^{t-s}, \quad 0 \leq t \leq 2 n, n \geq 1 。
$$

In (49) the binomial coefficients $\left(\begin{array}{l}x \\ y\end{array}\right)$ are understood to be zero unless $0 \leq y \leq x$, and $c_{\infty 0}=-p_{0}=-\sqrt{\frac{2}{\pi}}$.

We finally have from $(38),(8)$ and (47) the following asymptotic expansion

for $u$ :

(50) $u v \frac{e^{i k R}}{\sqrt{i k R}} \sum_{n=0}^{\infty} \rho_{n}(i k R)^{-n}+\frac{e^{i k(r+2 a)}}{\sqrt{i k r w}} \sum_{n=0}^{\infty}(2 i k a)^{-n} \sum_{j=0}^{n}\left(\frac{2 a}{r_{W}}\right)^{j} \sum_{t=0}^{2 n-j} c_{j t n^{w}}{ }^{-t}$.

The $c_{j t n}$ are given recursively by $(48)$ and $(49)$, beginning with $c_{000}=-\sqrt{\frac{2}{\pi}}$; the $\rho_{n}$ are defined in equation (39); and $w$ is defined in equation (44). The first few terms of the expansion for $u$ are, from (50),

$$
\begin{aligned}
u \sim e^{i k R} & \sum_{n=0}^{\infty} \rho_{n}(i k R)^{-n-(I / 2)}-e^{i k(r+2 a)} \sqrt{\frac{2}{\pi i k r w}} \\
\cdot & {\left[1+\frac{1}{10 i k a}\left\{1-w^{-1}\left(1-4 P+3 Q^{2}-3 P^{2}\right)+3 w^{-2}\left(Q^{2}-P^{2}\right)\right\}\right.} \\
& \left.+\frac{1}{8 i k r}\left\{4 \mathrm{Fw}^{-1}+3 w^{-2}\left(Q^{2}-P^{2}\right)\right\}+\cdots\right]
\end{aligned}
$$

This problem has not been solved previously, so far as we know. It applies to the scattering by a soft hyperbolic cylinder of sound from a line source if $u$ de- 
notes pressure, and to the scattering by a perfectly conducting hyperbolic cylinder of an electromagnetic wave from a line source if $u$ denotes the $z$-component of the electric field, which must be the only non-vanishing component.

Fxample 6. The Field of a Line Source at one Focus of a Hyperbolic Cylinder $\left(\frac{\partial u}{\partial v}=0\right)$ We consider the same problem as that of Example 5 except that we replace the boundary condition $u=0$ on the cylinder by the condition that the normal derivative of u vanish there, i.e.,

$$
\frac{\partial u}{\partial v}=0 \text { on } \quad r=r_{0}(\theta) \text {. }
$$

We proceed as before and obtain in place of (4l) the conditions

$$
\begin{gathered}
v_{0}\left(r_{0}, w\right)=R^{-1 / 2}=\left(\frac{w-1}{2 a w}\right)^{1 / 2} \\
v_{n}\left(r_{0}, w\right)=-\frac{\partial v_{n-1}}{\partial r}-\frac{Q^{2}-p^{2}+2 P w-w}{2 a} \frac{\partial v_{n-1}}{\partial w}+\frac{\rho_{n}+\left(n-\frac{1}{2}\right) \rho_{n-1}}{(2 a)^{n+(1 / 2)}(1-w-1)^{n+\frac{1}{2}}}
\end{gathered}
$$

As a first consequence of (53) we obtain (43) but with the minus sign on the righthand side replaced by a plus sign. Then proceeding as before we obtain ( 47 ) for $f_{j n}(w)$ and $(48)$ for $c_{j t n}(j \geq 0)$. However, in place of (49) we find that $c_{\text {otn }}$ is given by

$$
\begin{aligned}
c_{o t n}=(-1)^{t}\left(\begin{array}{l}
n \\
t
\end{array}\right)\left(\rho_{n}+\left[n-\frac{1}{2}\right] \rho_{n-1}\right) & -\sum_{j=1}^{n} \sum_{s=0}^{2 n-j} c_{j s n}\left(c_{t-s}^{j}\right)(-1)^{t-s} \\
+\sum_{j=0}^{n-1} \sum_{s=0}^{2 n-t-2} c_{j s, n-1}(-1)^{t-s} & {\left[\left(j+s+\frac{1}{2}\right)\left\{2 F\left(\begin{array}{c}
j \\
t-s
\end{array}\right)+\left(\begin{array}{c}
j \\
t-s+1
\end{array}\right)-\left(Q^{2}-p^{2}\right)\left(\begin{array}{c}
j \\
t-s-1
\end{array}\right)\right\}\right.} \\
& \left.-\left(j+\frac{1}{2}\right)\left(\begin{array}{c}
j+1 \\
t-s+1
\end{array}\right)\right], 0 \leq t \leq 2 n, n \geq 1 .
\end{aligned}
$$

As before, the asymptotic expansion of $u$ is given by (50) with the appropriate $c_{j t n}$ which are now determined from $(47)$ and (55) starting with $c_{\infty 0}=+\sqrt{\frac{2}{\pi}}$. The first few terms in the expansion of $u$ are, from (50), 
(56) $\begin{aligned} u & \sim e^{i k R} \sum_{n=0}^{\infty} \rho_{n}(i k R)^{-n-(1 / 2)}+e^{i k(r+2 a)} \sqrt{\frac{2}{\pi i k r w}}\left[1+\frac{1}{101 k a}\right. \\ & \left.\left\{1+8 P+w^{-1}\left(4 P+Q^{2}-P^{2}-5\right)+3 w^{-2}\left(Q^{2}-P^{2}\right)\right\}+\frac{1}{8 i k r}\left\{4 P w+3 w^{-2}\left(Q^{2}-P^{2}\right)\right\}+\cdots\right] \cdot\end{aligned}$

This problem has not been solved previously, so far as we know. It applies to the scattering by a rigid hyperbolic cylinder of sound from a line source if $u$ denotes pressure and to the scattering by a perfectly conducting hyperbolic cylinder of an electromagnetic wave from a line source if $u$ denotes the z-component of magnetic field, which must be the only non-vanishing magnetic field component.

Example 7. The Field of a Line Source over a Plane Interface

Let us consider space to be filled with two different media separated by the plane interface $y=0$. In the upper region $y>0, u$ is assumed to satisfy equation (1) while in the lower region $y<0$, $u$ is assumed to satisfy (I) with $k$ replaced by $k^{\prime}=\mu k$. Here $\mu$ is a positive constant which may be interpreted physically as the ratio $\frac{c_{+}}{c_{-}}$of propagation speed in the upper medium to that in the lower medium. At the interface $y=0, u$ is assumed to satisfy the two boundary conditions

$$
\begin{aligned}
& u\left(x, 0^{+}\right)=a u\left(x, 0^{-}\right) \\
& \frac{\partial u\left(x, 0^{+}\right)}{\partial y}=b \frac{\partial u\left(x, 0^{-}\right)}{\partial y} .
\end{aligned}
$$

In these conditions, $\mathrm{a}$ and $\mathrm{b}$ are two given constants. These conditions are appropriate to acoustic problems and certain electromagnetic problems.

In addition to the preceding conditions, the field $u$ is assumed to be produced by a line source which is parallel to the interface and normal to the

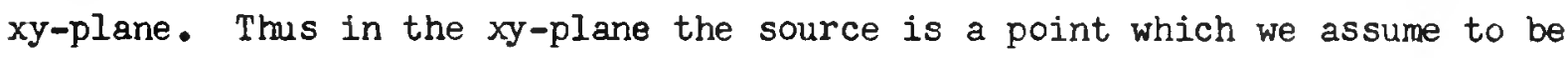




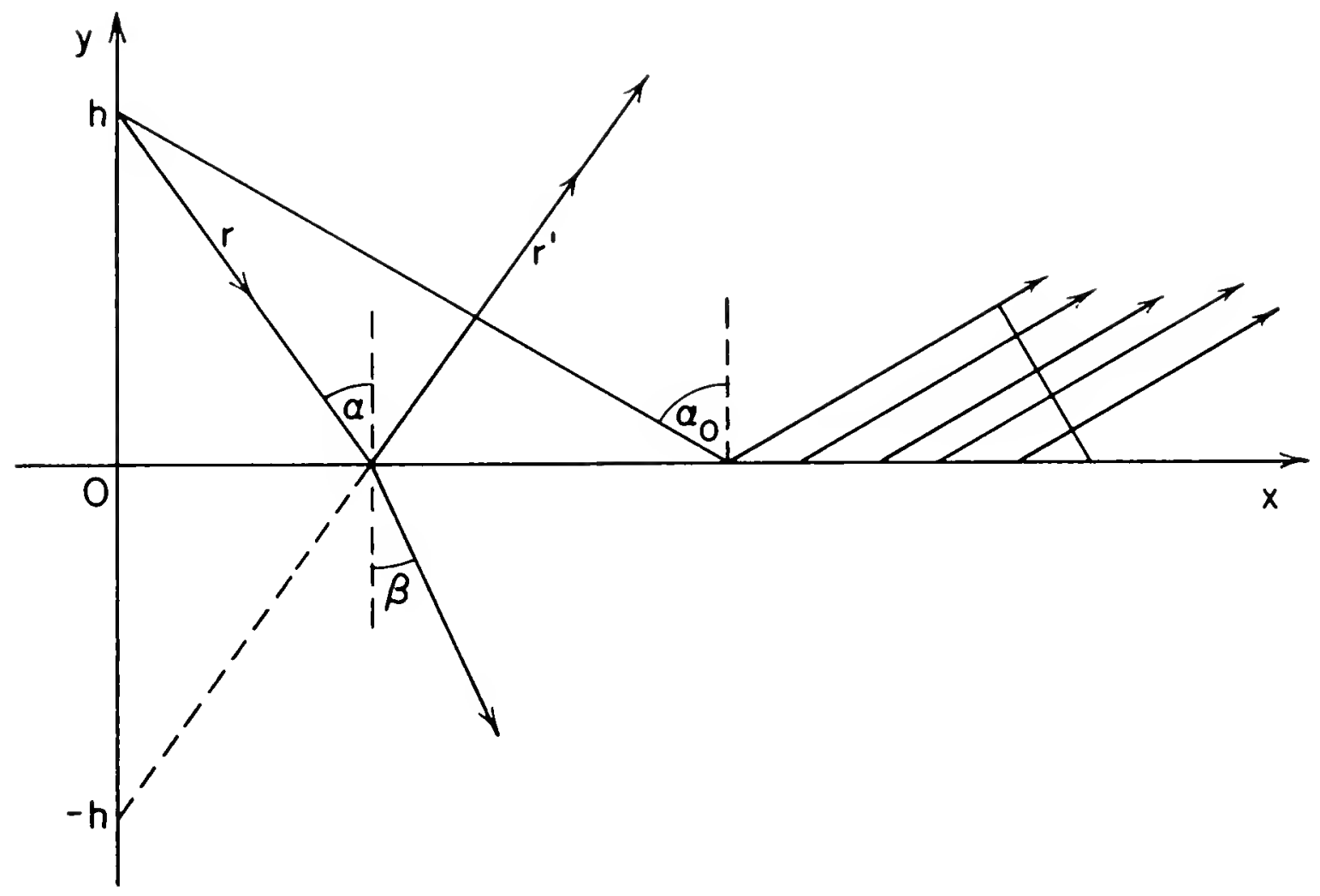

\section{Figure 5}

The field at a line (or point) source over a plane interface. The source is located in the upper half-space $y>0$ at $x=0, y=h$ (or at $x=z=0$, $y=h)$. The incident rays are lines radially directed from the scurce, and $a$ denotes the angle which a ray makes with the $y$-axis, which is the angle of incidence of the ray at the interface $y=0$. The reflected rays are lines which appear to radiate from the image source at $x=0, y=-h$ ( or at $x=z=0, y=-h_{1}$ ). The reflected rays enter the second medium in the lower half-space making the angle $\beta$, the angle of refraction, with the y-axis. If the propagation speed in the lower medium exceeds that in the upper medium there is a critical angle of incidence $a_{0}$ for which $\beta=\pi / 2$. The refracted ray is then along the interface, and from any point on it a diffracted ray may split off into the upper medium making the angle $a_{0}$ with the $y$-axis. The surface normal to these dif'racted rays is a diffricted waverront, which appears as a line segment in the figure. 
located at $x=0, y=h>0$ in the upper half-plane (see Figure 5). We are thus dealing with a two-dimensional problem in which the solution u consists cf a cylindrical wave coming from the source plus outgoing waves. The cylindrical wave is given exactly by (14) or asymptotically by (15), with $\mathrm{m}=0$ in both cases, according to the definition of a line scurce. We wish to determine the asymptotic expansion of the solution $u$.

As a first step in the construction of the asymptotic expansion, we assume that the expansion has the form

$$
u \sim e^{i k r} \sum_{n=0}^{\infty} \rho_{n}(i k r)^{-n-(I / 2)}+e^{i k I} \sum_{n=0}^{\infty}(i k)^{-n-(1 / 2)} v_{n}, y>0,
$$

$$
u \sim e^{i k^{\prime} \cdot} \sum_{n=0}^{\infty}\left(i k^{\prime}\right)^{-n-(I / 2)} w_{n},
$$$$
\mathrm{y}<0
$$

This assumption is based on the expectation that in the upper region $u$ will contain a reflected wave in additicn to the incident cylindrical wave, while in the lower region $u$ will consist entirely of a transmitted or refracted wave. This assumption will be justified below for $\mu \geq I$, i.e., for the case where no total reflection occurs. However, when total reflection does occur $(\mu<1)$, the preceding assumption must be modified by including a diffracted wave in the upper region and an evanescent wave in the lower region. These modifications will be considered after the case $\mu>1$ has been treated.

Upon inserting the assumed asymptotic expansion (59), (60) into the boundary conditions (57), (58) and equating coefficients of corresponding powers of $k$ the obtain

$$
\begin{aligned}
& \Psi(x, c)=\mu \Psi(x, 0)=r(x, 0)=\left(h^{2}+x^{2}\right)^{I / 2}, \\
& o_{n} r^{-11-(1 / 2)}+v_{n}(x, c)=a \mu^{-1-(I / 2)} w_{n}(x, 0), \\
& h r^{-n-(3 / 2)}\left(\left[n-\frac{1}{2}\right] \rho_{n-1}-\rho_{n}\right)+h r^{-1} v_{n}(x, 0)+\frac{\partial}{\partial y} v_{n-1}(x, 0) \text {, } \\
& =b \mu^{-n+(1 / 2)}\left[\frac{\partial \Psi^{\prime}}{\partial y}(x, 0) w_{n}(x, 0)+\frac{\partial}{\partial y} w_{n-1}(x, 0)\right], n=0,1, \cdots .
\end{aligned}
$$


The quantities $p_{-I}, v_{-1}$ and $w_{-1}$ are understood to ve zero in (63) and are introduced merely to unify the equations for $n=0$ and $n \neq 0$.

From (3) and (61), and from the condition that the second wave in (59) must be outgoing, we find

$$
\Phi(x, y)=r^{\prime}=\left[x^{2}+(y+h)^{2}\right]^{1 / 2} .
$$

In $(64), r^{\prime}$ denotes the distance from $(x, y)$ to the image source at $(0,-h)$; and the positive square root is intended. Thus the reflected rays satisfy the law of reflection and appear to come from the image source, and hence the reflected wave is also cylindrical. Therefore the $v_{n}$ are of the form $(8)$ with the $f_{i j}\left(\theta^{\prime}\right)$ determined by (9) and (10).

To determine $I$ ' we again use (3), (61), and the outgoing wave condition. We first note that (3) and (61) imply that the transmitted rays satisfy the law of refraction. In terms of the angle of incidence $a$ and the angle of refracticn $\beta$ this condition is

$$
\sin \beta=\mu^{-1} \sin \alpha \text {. }
$$

By making use of these angles, and by considering the variation in $Y^{\prime}$ along a $r e-$ fracted ray, we obtain paranetric equations for the wavefront ( $\mathbb{F}^{\prime}=$ constant) in the form

$$
\begin{aligned}
& y=-\left(Y^{\prime}-h \mu^{-1} \sec \alpha\right) \cos \beta, \\
& x=\left(1-h \mu^{-1} \sec \alpha\right) \sin \beta+h \tan a_{0}
\end{aligned}
$$

These equations determine $I^{\prime}(x, y)$. It is apparent that the refracted wave is not cylindrical if $\mu \neq 1$ and therefore the $w_{n}$ are given by (6), rather than by the simpler formulas for cy?indrical waves.

Now to compute the $v_{n}$ and $w_{n}$ at $y=0$ we solve $(62)$ and (63), obtaining $(68)$

$$
\begin{aligned}
w_{n}(x, 0)=\left\{r h^{-1}\left[b \mu^{-n+(1 / 2)} \frac{\partial w_{n-1}}{\partial y}-\frac{\partial v_{n-1}}{\partial y}\right]\right. \\
\left.\quad-r^{-n-(1 / 2)}\left[\left(n-\frac{1}{2}\right) \rho_{n-1}-2 \rho_{n}\right]\right\} \mu^{n+(1 / 2)}\left(a-r h^{-1} b \mu \frac{\partial y}{\partial y}\right)^{-1},
\end{aligned}
$$




$$
v_{n}(x, 0)=a \mu^{-n-(1 / 2)} w_{n}-p_{n} s^{-n-(1 / 2)}
$$

These boundary values together with equation (6) for the $w_{n}$ and (8) for the $v_{n}$ suffice for the successive calculation of the $v_{n}$ and $w_{n} \cdot$ From (7) and (68), we have at once

$$
v_{0}(x, y)=\frac{1-\bar{z}}{1+Z}\left(r^{\prime}\right)^{-1 / 2} ; \quad z=\frac{\mu b \cos \beta}{a \cos \alpha} .
$$

To find $w_{0}$ we use (69) and (6). The second term of (6) is zero, so the first term alone yields $w_{0}$. When $G(s)$ is obtained from equations (66) and (67) which determine the wavefront, we find

$$
w_{0}(x, y)=\frac{2}{1+2} \frac{(\sin \alpha)^{1 / 2} \cos \beta}{a \cos \alpha}\left[|x|-\left(\mu^{-2}-1\right) h \tan ^{3} \alpha\right]^{-1 / 2},
$$

In order to calculate $v_{1}$ we insert the above expressions for $v_{0}$ and $w_{0}$ into equation (68) and obtain for $v_{1}$ at $y=0$

$$
v_{1}(x, 0)=(1+Z)^{-1}\left\{\mathrm{rh}^{-1}\left(\mathrm{b \mu}^{-1 / 2} \frac{\partial \mathrm{w}_{0}}{\partial \mathrm{y}}-\frac{\partial \mathrm{v}_{0}}{\partial y}\right)-\left(8 \pi \mathrm{r}^{3}\right)^{-1 / 2}\right\}-\frac{1}{8}\left(\frac{2}{\pi r^{3}}\right)^{1 / 2} .
$$

When this boundary value of $v_{1}$ and equation (70) for $v_{0}$ are used in (9) and (10), we find

$$
v_{1}(x, y)=\left(\frac{h}{r \cos \alpha}\right)^{1 / 2} v_{I}(x, 0)+\frac{1}{2 r^{1 / 2}}\left(r^{-1}-h^{-1} \cos \alpha\right)\left(\frac{1}{4}+\frac{d^{2}}{d e^{2}}\right) \frac{1-z}{1+Z} .
$$

In a similar way but with more complicated calculation, $w_{1}$ and the sicceeding terms can be computec; however we will not determine any more of them here. The lowest-order terms $v_{0}$ and $w_{0}$ were previously found by $K .0$. Friedrichs and J.B. Keller [14]. It is apparent that the assumed form of asymptotic expansion (59) and (60) can be completely determined by the preceding methods, and that it satisfies the conditions of the problem. 
rays incident at angles greater than the critical angle $a_{0}=\sin ^{-1} \mu$. In this case the transmitted rays, corresponding to rays incident at angles between 0 and $a_{0}$, cover the entire lower half-plane. In particular the critically rePracted ray, for which $\beta=\frac{\pi}{2}$, lies in the interface $y=0$. Thus if any wave is produced in the lower half-plane by the totally reflected rays it is present in the same region as that occupied by the transmitted wave, and it wculd have to be included in the expansion of $u$. Without this additional wave we find that the boundary conditions at the interface cannot be satisfied on that portion of the interface where total reflection occurs. However, even if we add this wave in the lower region the boundary conditions still canrot be satisfied. It is necessary to add another wave in the upper region as we1l, and then the boundary conditions can be satisfied. Therefore we assume that $u$ has the asymptotic form (74) $u \sim e^{i k r} \sum_{n=0}^{\infty} \rho_{n}(i k r)^{-n-(1 / 2)}+e^{i k \Psi} \sum_{n=0}^{\infty}(i k)^{-n-(1 / 2)} v_{n}+e^{i k \phi} \sum_{n=0}^{\infty}(i k)^{-r_{1}-(1 / 2)} s_{n}$, $\mathrm{y}>\mathrm{O}$

(75) $u \sim e^{i k^{\prime} I^{\prime}} \sum_{n=0}^{\infty}\left(i k^{\prime}\right)^{-n-(1 / 2)} w_{n}+e^{i k \cdot x} \sum_{n=0}^{\infty}(1 k)^{-n-(1 / 2)} t_{n}, \quad y<0$.

We will call the waves in the upper region the incident, reflected and diffracted waves respectively, and in the lower region the transmitted or refracted wave and the evanescent wave. The reasons for this choice of names will become clear presently.

The interface may be divided into the region of regular reflection, for which $|x| \leq h \tan a_{0}$, and the region of total reflection $|x|>h$ tan $a_{0}$. In the region of regular reflection we will assume that only the incident, reflected, and refracted waves are present and that they satisfy the boundary conditions. In the region of total reflection we will assume that all the waves are present and that the incident, reflected, and evanescent waves together satisfy the boundary conditions while the transmitted and diffracted waves together also satisfy the 
boundary conditions. In this way the boundary conditions will be satisfied everywhere on the interface, provided that the diffracted and evanescent waves are really abeent in the ragion of regular reflection. This will be found to be the case when the solution is constructed.

On the basis of the foregoing method of satisfying the boundary conditions, we see that the transmitted and regularly reflected parts of the field are determined as before. Similarly the evanescent wave and the totally reflected part of the reflected field also satisfy equations (6I)-(63). The angle of refraction $\beta$ is complex for these waves, however, and therefore the evanescent wave cannot be determined in the same way as was the transmitted wave. Since the evanescent wave is not required for the determination of the term with $n=0$ in the totally reflected wave, this term can be found in exactly the same way as before. Once the evanescent wave is found, the other terms in the totally reflected wave can be found in the same way as can those in the regularly reflected wave. We will not consider these waves further, except to note that since $\beta$ is complex the evanescent wave is really evanescent (i.e., exponentially decaying) and can therefore be omitted in the region of regular reflection, where it is of an exponentially lower order than all the other waves.

We now turn to the determination of the diffracted wave, which together with the transmitted wave, has been assumed to satisfy the boundary conditions in the region of total reflection. The transmitted wave is in principle completely determined by the recursion formula (6) and the fact that, together with the incident and reflected waves, it satisfies the boundary conditions in the region of regular reflection. Therefore the remaining conditions in the region of total reflection serve to determine the diffracted wave in terms of the transmitted wave. We now insert the diffracted wave, givan by the last term in $(7 / 4)$ and the transmitted wave, given by the first term in (75), into the boundary conditions (57) and (58) in the region of total reflection, obtaining 
(76) $e^{1 k \emptyset} \sum_{n=0}^{\infty}(i k)^{-n-(1 / 2)} s_{n}=a e^{1 k^{\prime} I^{\prime}} \sum_{n=0}^{\infty}\left(i k^{\prime}\right)^{-n-(1 / 2)} w_{n}$, at $y=0,|x|>n \tan a_{0}$

(77) $e^{1 k \phi} \sum_{n=0}^{\infty}(i k)^{-n-(1 / 2)}\left[i k \frac{\partial \phi}{\partial y} s_{n}+\frac{\partial s_{n}}{\partial y}\right]$

$$
=b e^{i k^{\prime} Y^{\prime}} \sum_{n=0}^{\infty}\left(i k^{\prime}\right)^{-n-(1 / 2)}\left[i k^{\prime} \frac{\partial Y^{\prime}}{\partial y} w_{n}+\frac{\partial w_{n}}{\partial y}\right] \text {, at } y=0,|x|>n \tan a_{0} \cdot
$$

Upon equating coefficients of corresponding powers of $k$ in these two equations, noting that $k^{\prime}=\mu k$, we obtain

(80) $\quad i \frac{\partial \phi}{\partial y} s_{n+1}+\frac{\partial s_{n}}{\partial y}=b \mu^{-n-(1 / 2)} \frac{\partial w_{n}}{\partial y}$.

In deriving (80) we have used the fact that $\frac{\partial Y^{\prime}}{\partial y}=0$ at $y=0$ since the wavefronts $\left(\Psi^{\prime}=\right.$ constant) are orthogonal to the plane $y=0$.

From (76), (3) and the outgoing wave condition, we have

(81) $\phi(x, y)=(h+y) \sec a_{0}+\mu\left[|x|-(h+y) \tan a_{0}\right], y \geq 0,|x| \geq(h+y) \tan a_{0} \cdot$

The wavefronts given by (81) are parallel straight lines and the rays are consequently the parallel lines which make the angle $a_{0}$ with the y-axis (see Figure 5). Thus the diffracted wave is a general plane wave of the type to be considered in section VI, 1.e., the wavefronts are parallel planes but the amplitude on each plane is not constant. SInce (79) determines $\delta_{n}$ at $y=0$ in terms of $w_{n}$, which is already completely determined, the formulas of section 6 can be used to calculate $s_{n}$ off the plane $y=0$. For the first term $s_{0}$ the result is, since $w_{0}-0$ at $g=0$,

$$
8_{0}=0
$$

The next term $s_{1}$ is then constant along each ray, and hence 
(83) $s_{1}(x, y)=a \mu^{-3 / 2} w_{1}\left(x-y \tan a_{0}, 0\right), \quad x>0, y>0, \quad x-y \tan a_{0} \geq h \tan a_{0}$, (84) $s_{1}(x, y)=a \mu^{-3 / 2} w_{1}\left(x+y \tan a_{0}, 0\right), \quad x<0, \quad y>0, \quad x+y \tan a_{0} \leq-h \tan a_{0}$. As we have not yet computed $w_{1}$ explicitly, we note that $s_{1}(x, 0)$ can also be found from ( 80$)$ with $n=0$. Thus, noting that $\frac{\partial \phi}{\partial y}=\cos a_{0}$, we have instead of (83)

(85) $s_{1}(x, y)=i b \mu^{-1 / 2} \sec a_{0} \frac{\partial w}{\partial y}\left(x-y \tan \alpha_{0}, 0\right), \quad x>0, \quad y>0, \quad x-y \tan \alpha_{0} \geq h \tan \alpha_{0} \cdot$ An equation similar to $(84)$ applies for $x<0$. If we compute $\frac{\partial w_{0}}{\partial y}$ from (71) we obtain for $s_{1}$ from $(85)$

$$
s_{1}(x, y)=-\frac{2 b\left(\sin a_{0}\right)^{1 / 2}}{a \cos ^{2} a_{0}}\left[x-(y+h) \tan a_{0}\right]^{-3 / 2}, \quad x-(y+h) \tan a_{0}>0 .
$$

The term $e^{i k \phi}(i k)^{-3 / 2} s_{1}$ is the leading term in the diffracted field, since $s_{0}=0$. This leading term is of order $\frac{l}{k}$ with respect to the incident fleld. Its wavefronts are parallel planes, and its rays are parallel straight lines which may be interpreted as having originated from the incident ray with angle of incidence $a_{0}$. This critically incident ray gives rise to a refracted ray lying in the interface, and this refracted ray in turn splits into a family of diffracted rays all of which leave the interface at the critical angle $a_{0}$. These rays are given by a modified form of Fermat's priniple [6]. From (86) we see that the amplitude of $s_{1}$ is proportional to $\left[x-(y+h) \tan a_{0}\right]^{-3 / 2}$, where the expression in brackets is just the length of that part of the ray from the source to $x, y$ which lies in the interface. Thus $s_{1}$ becomes infinite on that ray for which this length is zero, which is just the critical totally reflected ray. Therefore the present asymptotic expansion fails on that ray. For fixed $y$ and large $x,{ }_{1}$ behaves like $x^{-3 / 2}$, which is a more rapid decay than that of the incident cylindrical wave. The term $s_{1}$ was also obtained 
by K. O. Friedrichs and J. B. Keller $[14]$. However, they did not determine $v_{1}$, which 1 s of the same order as $s_{1}$.

In summary, we have found that when $\mu \geq 1$, no total reflection occurs and the asymptotic expansion consists of one incident and one reflected cylindrical wave in the upper medium, and of a single transmitted wave in the lower medium. The waves in the upper medium are given by (59), and the wave in the lower medium by $(50)$. The incident wave is completely known, the first two terms of the reflected wave have been computed $\left[v_{0}\right.$ is given in $(70), v_{1}$ in $(73)$ and $\mathbf{I}$ in $\left.(64)\right]$, and the first term of the transmitted wave has been computed [ $w_{0}$ is given in (71) and 1 in $(66),(67)]$. Additional terms can be computed making use of (68), (69) and the formulas of section 2 .

In the case $\mu<1$, total reflection occurs for rays incident at angles, greater than the critical angle $a_{0}=\sin ^{-1} \mu$. In this case the asymptotic expansion in the upper medium ccntains a diffracted wave in addition to the incident and reflected cylindrical waves. This diffracted wave is a general plane wave and is not present in that part of space covered by regularly reflected rays, but only in the region covered by totally reflected rays. It may be thought of as being produced by the transmitted wave, which travels along the interface and 'leaks' back into the upper medium. This wave is given by the third series in (74). Only its first two terms have been computed, the first of which ( $s_{0}$ ) is zero and the second of which can be found from equations (86) for $s_{1}$ and (81) for $\varnothing$.

In the lower medium an evanescent wave occurs in addition to the ordinary transmitted wave. This wave has not been determined although its boundary value at $y=0$ has been found. The reflected and transmitted waves are given by the same formulas as in the case $\mu \geq 1$.

Finally it must be pointed out that although (79) completely determines the boundary values $\varepsilon_{n}(x, 0)$ of the terms in the diffracted wave, equation $(80)$ must also be satisfied. Since all the terms in ( 80 ) are already determined, this equation must be an identity. 
4. General Cylindrical Waves

By a general or three-dimensional cylindrical wave we mean a wave with concentric circular cylinders as wavefronts but with an anplitude depending upon all three space variables. If the axis of the cylinders is the z-axis then the the coefficients $v_{n}$ will depend upon $z$ as well as upon $r$ and $\theta$, whereas for the usual or two-dimensional cylindrical waves, such as those in Section 3 , the $\nabla_{n}$ are independent of $z$. For general cylindrical waves equation (5) yields

$$
v_{0}(r, \theta, z)=\frac{g_{0}(r, \theta, z)}{r^{1 / 2}} \text {. }
$$

From equations $(6)$ and $(87)$ we find by induction that $v_{n}$ has the form

$$
v_{n}(r, \theta, z)=\sum_{i=-n}^{n} \sum_{j=0}^{n} a_{i j n} r^{-(1 / 2)-i}(\log r)^{j} .
$$

Thus the asymptotic expansion of a general cylindrical wave is

$$
u \sim \frac{e^{ \pm i k r}}{\sqrt{r}} \sum_{n=0}^{\infty}(i k)^{-n} \sum_{\substack{i=-n \\ j=0}}^{\substack{j=n \\ j=n}} a_{i j n} r^{-i}(\log r)^{j} \text {. }
$$

Insertion of (88) into (6) yields a rather complicated recursion formula for the $a_{i j n}$. Therefore instead of giving this formula, we list below the expressions for those $a_{i j n}$ with $n=1$ and 2 which are not zero. For $n=1$ we find

$$
\begin{aligned}
& a_{101}=\frac{1}{2}\left[\frac{1}{4} g_{0}(\theta, z)+D_{\theta}^{2} g_{0}\right], \\
& a_{001}=-\frac{1}{2 r_{0}}\left[\frac{1}{4} g_{0}(\theta, z)+D_{\theta}^{2} \cdot g_{0}\right]+\frac{r_{0}}{2} D_{z}^{2} g_{0}+r_{0}^{1 / 2} v_{1}\left[r_{0}, \theta, z\right], \\
& a_{-1,01}=-\frac{1}{2} D_{z}^{2} g_{0} .
\end{aligned}
$$

Thus

$$
v_{1}(r, \theta, z)=a_{101} r^{-3 / 2}+a_{001} r^{-1 / 2}+a_{-1,01} r^{1 / 2}
$$


Similarly for $n=2$

$$
\begin{aligned}
a_{202}= & \frac{1}{4}\left(\frac{9}{4}+D_{\theta}^{2}\right) a_{101}, \\
a_{102}= & \frac{1}{2}\left(\frac{1}{4}+D_{\theta}^{2}\right) a_{001}, \\
a_{012}= & -\frac{1}{2}\left\{\left[\frac{1}{4}+D_{\theta}^{2}\right] a_{-1,01}+D_{z}^{2} a_{101}\right\}, \\
a_{002}= & -\sum_{i=0}^{1} \frac{r_{0}^{-1-i}}{2 i+2}\left(\left(i+\frac{1}{2}\right)^{2}+D_{\theta}^{2}\right) a_{i 01}+\sum_{i=0}^{1} \frac{r_{0}^{i+1}}{2 i+2} D_{z}^{2} a_{-i, 01} \\
& +\frac{1}{2}\left\{\left(\frac{1}{4}+D_{\theta}^{2}\right) a-1,01+D_{z}^{2} a_{101}\right\} \log r_{0}+r_{0}^{1 / 2} v_{2}\left[r_{0}, \theta, z\right], \\
a_{-1,02}= & -\frac{1}{2} D_{z}^{2} a_{001}, \\
a_{-2,02}= & -\frac{1}{4} D_{z}^{2} a_{-1,01} .
\end{aligned}
$$

Thus

$$
\begin{aligned}
& v_{2}(r, \theta, z)=a_{202} r^{-5 / 2}+a_{102} r^{-3 / 2}+a_{012} r^{-1 / 2} \log r \\
& +a_{002} r^{-1 / 2}+a_{-1,02} r^{1 / 2}+a_{-2,02} r^{3 / 2} \text {. }
\end{aligned}
$$

General cylindrical waves may occur in the diffraction of a plane wave by a wedge whose properties depend upon 2 (a stratified wedge), in the diffraction of a general plane wave by a parabolic cylinder, etc. However, we will not consider here any examples involving such waves. It should be noted from (88) that the $v_{n}$ with $n \geq I$ contain positive powers of $r$ and $\log r$, and therefore become infinite as $r$ does. This shows that the asymptotic expansion (89) for a general cylindrical wave is not uniform in $r$. These observations will be related to corresponding results for the asymptotic expansions of arbitrarily shaped waves in Section 


\section{Spherical waves}

We will now consider waves whose wavefronts are concentric spheres; the rays are ther lines emanating from a point. The Gaussian curvature of the wavefront $r=$ constant is $G(r)=r^{-2}$ and the phase $I$ is given by $\Psi= \pm r+$ constant. Again we may set the constant equal to zero without loss of generality. Now, using $r$ instead of $s$, we have from (6)

$$
v_{0}(r, \theta, \phi)=\frac{g_{0}(\theta, \phi)}{r} \text {. }
$$

The factor $g_{0}(\theta, \phi)=r_{0}(\theta, \phi) v_{0}\left[r_{0}(\theta, \phi), \theta, \phi\right]$ is constant on the ray $\theta=$ const., $\phi=$ const.

$$
\text { Using (92) in (6) we find, by induction, that } v_{n}(r, \theta, q) \text { can be written }
$$
in the form

$$
v_{n}(r, \theta, \phi)=\sum_{j=0}^{n} f_{j n}(\theta, \phi) r^{-j-1}
$$

Now inserting (93) into (6) yields the following recursive formulas for $f_{j n}(\theta, \emptyset)$ :

$$
\begin{array}{ll}
f_{j n}= \pm \frac{1}{2 j}[j(j-1)+B] f_{j-1, n-1}, \quad j \neq 0, & n \geq 1, \\
f_{\text {on }}=r_{0}(\theta, \phi) v_{n}\left[r_{0}(\theta, \phi), \theta, \phi\right]-\sum_{j=1}^{n} r_{0}^{-j} f_{j n}, & n \geq 1 .
\end{array}
$$

In equations (94) $B$ is the Beltrami operator $B=\frac{1}{\sin \theta}\left[\frac{\partial}{\partial \theta} \sin \theta \frac{\partial}{\partial \theta}+\frac{1}{\sin \theta} \frac{\partial^{2}}{\partial \phi^{2}}\right]$ and the upper sign is to be used if $Y=+r$ while the lower sign is to be used if $\Psi=-r$. The function $f_{00}$ is defined by $f_{00}=g_{0}(\theta, \varphi)$, and $v_{n}\left(r_{0}, \theta, \varphi\right)$ is the value of $v_{n}$ at the point $v_{0}$ on the ray $\theta=$ const., $\phi=$ const. Using (93) in (2) we obtain as the asymptotic expansion of a spherical wave

$$
u \sim \frac{e^{ \pm i k r}}{r} \sum_{n=0}^{\infty}(i k)^{-n} \sum_{j=0}^{n} f_{j n}(\theta, \phi) r^{-j} .
$$


If the $v_{n}\left[r_{0}(\theta, \phi), \theta, \phi\right]$ are given, then the $f_{i n}(6, \varphi)$ can be computed successively from (94) and (95), and then the expansion of $u$ is given by (96). For example, if the expansion of $u$ is given on a surface $r=r_{0}(\theta, \phi)$ then all the $v_{n}$ are given on that surface, and therefore the expansion everwhere is determined. We will now consider certain applications of these results.

Example 8. Diffraction of a Plane Wave by a Cone of Arbitrary Cross-Section In the diffraction of a plane wave by a cone, the asymptotic expansion contains a spherical wave centered at the vertex, in addition to other waves. The preceding considerations apply to this spherical wave. Since the problem contains no characteristlc dimension, $k$ and $r$ can occur only in the combination $\mathrm{kr}$. To achieve this dependence in the asymptotic expansion (2) we first multiply (2) by $\mathrm{k}^{-1}$, which we may do since (2) is linear and homcgeneous. Then since the coefficient of $v_{n}$ is $k^{-n-1}$, all $f_{\text {in }}$ must be zero except $f_{n n}$, as we see from (93). Thus we have, choosing $\Psi=+r$ in order to obtain an outgoing ware,

$$
v_{n n}\left(r_{, \theta}, \phi\right)=f_{n n}(\theta, \phi) r^{-n-I}
$$

The $f_{n n}$ can be found successively from $f_{00}=g_{0}(\theta, \phi)$ by means of $(94)$. From this equation we obtatn

$$
f_{n n}(\theta, \phi)=\frac{1}{2^{n} n !}\left\{\prod_{j=1}^{n}[j(j-1)+B]\right\} g_{0}(\theta, \phi), \quad n \geq 1 .
$$

Collecting our result $\varepsilon$, we have

$$
u \sim \frac{e^{i k r}}{k r}\left[1+\sum_{n=1}^{\infty} \frac{1}{(2 i k r)^{n} n !}\left\{\prod_{j=1}^{n}[j(j-1)+B]\right\}\right] g_{0}(\theta, \varphi) .
$$

Here as in the wedge problem of Example 1, the present method does not yield $g_{0}(\theta, \varphi)$, but once $g_{0}$ is found in some other way, it yields the complete expansion of the spherical wave $u$. 
Example 9. Spherical Bessel Functions

Spherical wave solutions of (l) are given by the product of $(\mathrm{kr})^{-1 / 2}$ with a spherical Bessel function $H_{m+(1 / 2)}^{(1)}(k r)$ and a spherical harmonic $Y_{m}(\theta, \phi)$ of degree $m$. Thus

$$
u=(k r)^{-1 / 2} H_{m+(1 / 2)}^{(1)}(k r) Y_{m}(\theta, \varphi) \text {. }
$$

Since this solution depends upon $\mathrm{k}$ and $\mathrm{r}$ only in the combination $\mathrm{kr}$, its expansion is of the form (99) with $g_{0}=A Y_{m}$, where $A$ is a suitable constant, and with $Y=+r$ since (100) represents an outgoing wave. The result of applying the Beltrami operator to a spherical harmonic is $B Y_{m}=-m(m+1) Y_{m}$ and thus the $B$ in (99) can be replaced by $-m(m+1)$. But then the sum terminates with the term $\mathrm{n}=\mathrm{m}$, as all later products will contain the factor $(\mathrm{m}+1) \mathrm{m}+\mathrm{B}$, which is zero. Thus the expansion is finite and therefore yields an exact expression for $(k r)^{-1 / 2} H_{m+(1 / 2)}^{(1)}(k r)$ which is

$$
(k r)^{-1 / 2_{H}(1)}{ }_{m+(1 / 2)}(k r)=A \frac{e^{i k r}}{k r}\left\{1+\sum_{n=1}^{m} \frac{1}{(2 i k r)^{n} n !} \prod_{j=1}^{n}[j(j-1)-m(m+1)]\right\} \text {. }
$$

This expression for the spherical Bessel function agrees exactly with the known representation if $\mathrm{A}=i^{-\mathrm{n}-1}\left(\frac{2}{\pi}\right)^{1 / 2}$ (see $[20], \mathrm{p} \cdot 78$, eq. 3). If we had chosen $Y=-r$ we would have obtained the expansion of the incoming spherical Bessel function $\mathrm{H}_{\mathrm{m}+(1 / 2)}^{2}(\mathrm{kr})$, this expansions differs from the above merely in having $-i$ everywhere in place of $i$. (See $[20], p .78$, eq. 4).

Example 10. Diffraction of a Plane Wave by a Paraboloid of Revolution ( $u=0$ ) Let us consider a plane wave $e^{-i k x}$ incident from tho right along the axis of a parabcloid of revolution, and on the outside of it (see Fig. 1). This problem is similar to Example 3, except that the reflected wave is now spherical instead of cylindrical. If we let $r$ denote distance from the focus, we may write the equation of the paraboloid in the form (16). Again assuming $u=0$ on the 
boundary we obtain (17) and from it (18) - (20). The phase $Y$ is again given by (21), but instead of (22) we find from (92) that

$$
v_{0}(r, \theta)=-p^{-1} \sec ^{2} \frac{\theta}{2} \text {. }
$$

We see that $\nabla_{0}$ is independent of $\varnothing$ and therefore 80 are all the other $\nabla_{n}$, as was to be expected from the symmetry of the problem.

Now using (102) and the boundary conditions (20) in the recursion formulas (94) and (95) for the $f_{j n}(\theta)$, we find - and prove by induction - that $f_{j n}(\theta)$ is given by

$$
f_{j n}(\theta)=a_{j n} p^{j-n+1}\left(\sec ^{2} \frac{\theta}{2}\right)^{j+1}
$$

The $a_{j n}$ are given by the recursion formulas

$$
\begin{array}{ll}
a_{j n}=\frac{j}{2} a_{j-1, n-1}, & j \geq 1, n \geq 1, \\
a_{o n}=-\sum_{j=1}^{n} a_{j n}, & n \geq 1, \\
a_{o 0}=-1 . &
\end{array}
$$

Inserting equations (103) for the $f_{j n}(\theta)$ into (96), we obtain as the asymptotic expansion of $u$

$$
u \sim e^{-i k x}+e^{i k(r-2 p)} \sum_{n=0}^{\infty}(i k p)^{-n} \sum_{j=0}^{n} a_{j n}\left(p r^{-1} \sec ^{2} \frac{\theta}{2}\right)^{j+1} .
$$

By computing the first few $a_{j n}$ from (104) - (106) we obtain for the first few terms of (107)

$$
u \sim e^{-i k x}-e^{i k(r-2 p)} r^{-1} p \sec ^{2} \frac{\theta}{2}\left[1-\frac{1}{2 i k p}\left(1-\frac{p \sec ^{2} \frac{\theta}{2}}{r}\right)+\cdots\right]
$$

This problem has been solved exactly by H. Lamb $[18]$ and the asymptotic expansion of his solution is precisely (107). The amplitude and phase of the scattered wave given by his exact solution are compared with those given by (107) in Figures 6 and 7 . 


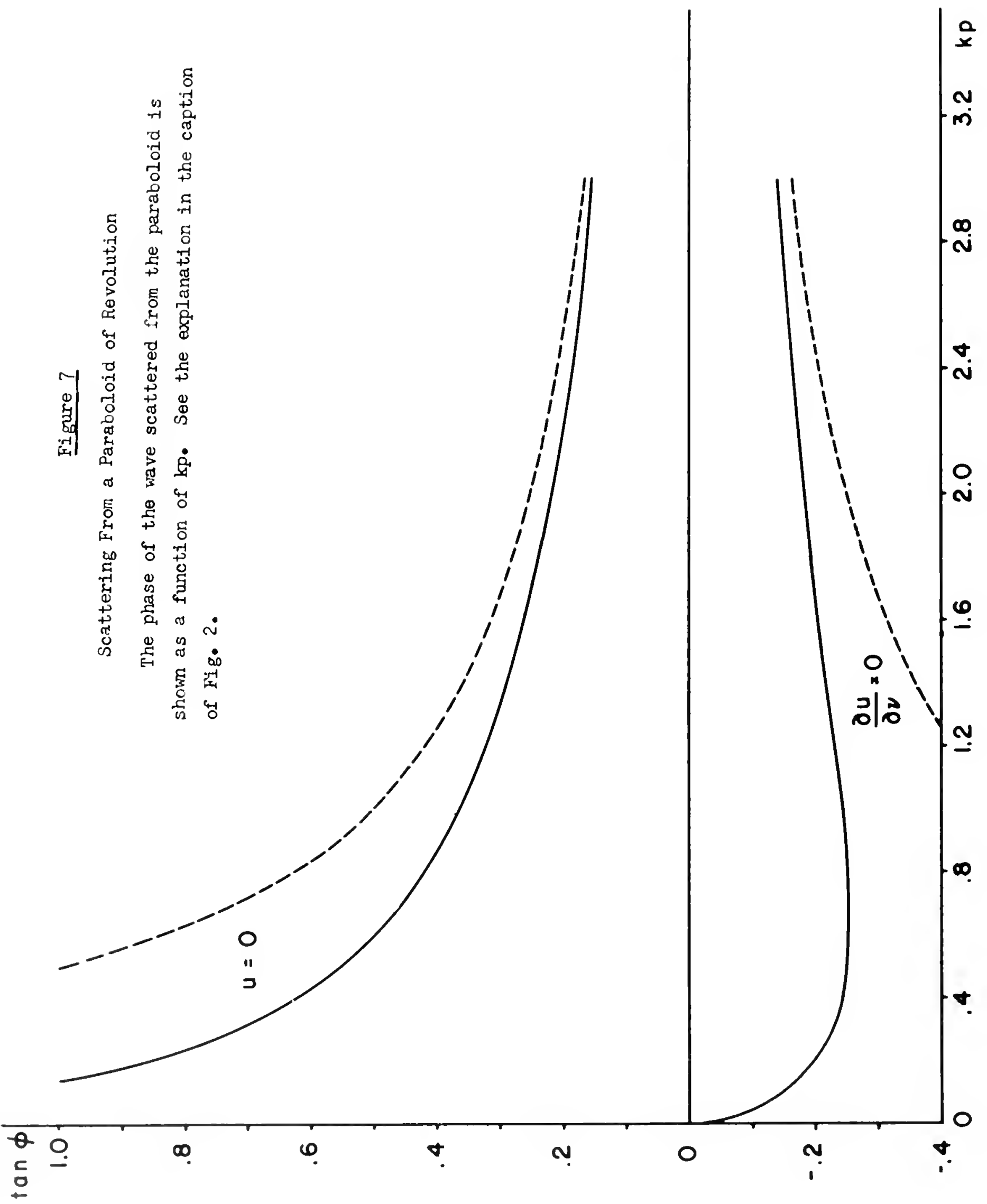


Example 11. Diffraction of a Plane Wave by a Paraboloid of Revolution $\left(\frac{\partial u}{\partial n}=0\right)$ We consider again the preceding problem except with the boundary condition $\frac{\partial u}{\partial n}=0$ on the paraboloid, instead of $u=0$ as in Example 10. This problem Is similar to the two-dimensional case treated in Example 4, and it can be treated in the same way. The first difference in the treatments occurs in the expression for $\nabla_{0}$, which is, instead of (35),

$$
v_{0}(r, \theta)=\frac{r_{0}(\theta)}{r}
$$

Now, using this expression for $v_{0}$ and the recursion formulas (94) and (95) for the $f_{j n}$ we find that the $f_{j n}$ are given by (103). The $a_{j n}$ satisfy the recursion formulas (104) - (106) with the minus signs in (105) and (106) replaced by plus signs. The asymptotic expansion of $u$ is given by (107) with the appropriate $a_{j n}$. The first few terms in this expansion are

$$
u \sim e^{-i k x}+e^{i k(r-2 p)} r^{-1} p \sec ^{2} \frac{\theta}{2}\left[1+\frac{1}{2 i k p}\left(1+\frac{p \sec ^{2} \frac{\theta}{2}}{r}\right)+\cdots\right] .
$$

This problem has also been solved exactly by $H$. Lamb $[18]$ and the asymptotic expansion of his solution is just (107) with the appropriate $a_{\text {jn }}$ In Figures 6 and 7 the exact amplitude and phase of the scattered wave are compared with the results of the asymptotic expansion given by (107).

Example 12. The Field of a Point Source at one Focus of a hyperboloid of hevolution $(u=0)$

Let us consider the field of a point source at one focus of a hyperboloid of revolution (see Fig. 4). This problem is similar to Example 5, except that spherical waves occur instead of cylindrical ones. Thus we assume instead of (38) that u has the expansion 


$$
u \sim \frac{e^{1 k R}}{R}+e^{i k Y} \sum_{n=0}^{\infty}(i k)^{-n} v_{n}(r, \theta, \phi) \text {. }
$$

Here, as before, $R$ denotes distance from the source. From the boundary condition on the hyperboloid we obtain (40) and, instead of (4l), the following:

$$
v_{0}=-R_{0}^{-1}, \quad v_{n}=0, n \geq 1, \quad \text { on } r=r_{0}(\theta) \text {. }
$$

The notation is the same as that of Example 5. Continuing, we obtain ( 42 ) for Y, but instead of (43) we find

$$
v_{0}(r, \theta)=-(w r)^{-1} \text {. }
$$

Again we note that the solution will be independent of $\phi$, and we consider the subsequent $f_{j n}$ as functions of $w$ rather than of $\theta$. Then in the recursion formulas $(94)$ and $(95)$ the operator $B$ must be expressed in terms of $w$ as $B=\left(Q^{2}-F^{2}+2 P w-w^{2}\right) \frac{\partial^{2}}{\partial w^{2}}$. We find that the $f_{j n}(w)$ have the form

$$
f_{j n}(w)=(2 a)^{j-n} \sum_{t=0}^{2 n-j} c_{j t n^{w-j-t-1}}
$$

The coefficients $c_{j t n}$ are determined by the recursion formulas

$$
\begin{aligned}
& c_{j t n}=\frac{1}{2 j}\left[(1-t-2 j) t c_{j-1, t+1, n-1}+2 P(j+t-1)^{2} c_{j-1, n-1}\right. \\
& \left.+\left(Q^{2}-P^{2}\right)(j+t-2)(j+t-1) c_{j-1, n-1}\right], \quad 1 \leq j \leq n, \\
& c_{\text {otn }}=-\sum_{j=1}^{n} \sum_{s=0}^{2 n-j} c_{j s n}(\underset{t-s}{j})(-1)^{t-s}, \quad 0 \leq t \leq 2 n, \quad n \geq 1 .
\end{aligned}
$$

From (113) wo see that $c_{000}=-1$.

Using the expression (114) for the $f_{j n}$ in (96) we have for the asymptotic exnansion of $u$ 
(117)

$$
u \sim \frac{e^{i k R}}{R}-\frac{e^{i k(r+2 a)}}{r w} \sum_{n=0}^{\infty}(2 i k a)^{-n} \sum_{j=0}^{n}\left(\frac{2 a}{r w}\right)^{j} \sum_{t=0}^{2 n-j} c_{j t n} w^{-t} .
$$

The first few terms in this expansion are

$$
\begin{aligned}
u \sim \frac{e^{i k R}}{R}-\frac{e^{i k(r+2 a)}}{r w}\left[1-\frac{1}{2 k a}\{P\right. & +\left(Q^{2}-P^{2}-P\right) w^{-1}-\left(Q^{2}-P^{2}\right) w^{-2} \\
& \left.\left.-P r^{-1}-1-\left(Q^{2}-P^{2}\right) r^{-1}-2\right\}+\cdots\right] .
\end{aligned}
$$

Example 13. The Field of a Point Source at one Focus of a Hyperbolold of Revolution $\left(\frac{\partial u}{\partial u}=0\right)$

We will now reconsider the previous example, choosing as boundary condition $\frac{\partial u}{\partial v}=0$ on the hyperboloid, fust as in Example 6, rather than $u=0$. Proceeding as in Examples 12 and 6 we find from the boundary conditions instead of (112)

$$
\begin{gathered}
v_{0}=R_{0}^{-1} \quad \text { at } r=r_{0} \\
v_{n}=-\left(\frac{\partial T}{\partial V}\right)^{-1}\left(\frac{\partial v_{n-1}}{\partial V}\right)=-\frac{\partial v_{n-1}}{\partial r}-\left(Q^{2}-P^{2}+2 P w-w^{2}\right)(2 a)^{-1} \frac{\partial v_{n-1}}{\partial w} \text {, at } r=r_{0} \cdot
\end{gathered}
$$

The $f_{j n}$ are then found to have the same form as before and the $c_{j t n}$ satisfy the same recursion formula when $j \neq 0$. However for $j=0$ we have, instead,

$$
\begin{aligned}
c_{o t n}= & -\sum_{j=1}^{n} \sum_{s=0}^{2 n-j} c_{j s n}\left(\begin{array}{c}
j \\
t-s
\end{array}\right)(-1)^{t-s}+\sum_{j=0}^{n-1} \sum_{s=0}^{2 n-j-2} c_{j s, n-1}(-1)^{t-s+1}\left[(1+j)\left(\begin{array}{c}
j+1 \\
t-s+1
\end{array}\right)\right. \\
& \left.+(j+s+1)\left\{\left(Q^{2}-P^{2}\right)\left(\begin{array}{c}
j \\
t-s-1
\end{array}\right)-2 P\left(\begin{array}{c}
j \\
t-s
\end{array}\right)-\left(\begin{array}{c}
j \\
t-s+1
\end{array}\right)\right\}\right] 0 \leq t \leq 2 n, \quad n \geq 1 .
\end{aligned}
$$

From (II9) we see that $c_{600}=+1$.

The expansion of $u$ is again given by (117) with the $c_{j t n}$ determined by 
(115) and (121) with $c_{000}=+1$. The first few terms are (122) $\begin{aligned} u \sim \frac{e^{i k R}}{R}+\frac{e^{i k(r+2 a)}}{w W}\left[1+\frac{1}{2 k a}\left\{P-2+(P+1) W^{-1}\right.\right. & +\left(Q^{2}-P^{2}\right) W^{-2}+P r^{-1}-1 \\ & \left.\left.+\left(Q^{2}-P^{2}\right) r^{-1}-2\right\}+\cdots\right] .\end{aligned}$

Example 14. The Fleld of a Point Source Over a Plane Interface

We will row reconsider Example 7, but replace the line source by a point source at $y=h, x=z=0$. Thus we must find the field produced by a point source situated in the upper medium ( $y>0)$, when the lover halfspace $(y<0)$ contains a different medium. At the interface $y=0$ we impose the two conditions (57), (58) on $u$ and $\frac{\partial u}{\partial y}$. The point source is assumed to produce a spherical wave $e^{i k r} / r$, and this wave is incident upon the interface, where it is reflected, transmitted and possibly diffracted. Thus we seek an asymptotic expansion for $u$ of the form

$$
u \sim \frac{e^{i k r}}{r}+e^{i k r} \sum_{n=0}^{\infty}(i k)^{-n_{v}}, \quad y>0
$$

$$
u \sim e^{i k \cdot I^{\prime}} \sum_{n=0}^{\infty}\left(i k^{\prime}\right)^{-n_{w_{n}}}, \quad y<0 .
$$

We call the waves in the upper medium the incident and reflected waves and that in the lower medium the transmitted wave. The constant $k^{\prime}=\mu k$ replaces $k$ in the lower medium. The above form of solution will be found to be valld If $\mu \geq 1$ in which case no total reflection occurs; the case $\mu<1$ will be treated later.

From the symmetry of the problem it is clear that the solution will depend upon $u$ and $\left(x^{2}+z^{2}\right)^{1 / 2}$ only, i.e., it will be symmetric about the $y$ axis. Therefore we will denote $\left(x^{2}+z^{2}\right)^{1 / 2}$ by $x \geq 0$ and consider $u$ as a 
function of $y$ and $x$. Now if we insert the assumed form of solution into the boundary conditions (57), (58) and equate coefficients of corresponding powers of $k$ we obtain (6I) and

$$
\left\{\begin{aligned}
r^{-1}+v_{0}(x, 0) & =a w_{0}(x, 0), \\
v_{n}(x, 0) & =a w_{n}(x, 0) \mu^{-n}, \quad n \geq 1,
\end{aligned}\right.
$$

$$
\begin{cases}-h r^{-2}+h r^{-1} v_{0}=b \mu w_{0}^{\prime} & \\
h r^{-3}+h r^{-1} v_{1}+v_{o y}=b\left[\begin{array}{ll}
y & w_{1}+w_{o y}
\end{array}\right], & (y=0) \\
h r^{-1} v_{n}+v_{n-1, y}=b\left[\mu_{y}^{1-n_{v_{1}}} w_{n}+w_{n-1, y}\right. & n \geq 2 .\end{cases}
$$

Then as before we obtain (64) for $\Psi(x, y)$, which shows that the reflected wave is also apherical, as was to be expected. We also obtain (65), (66) and (67) which define the angle of refraction and yield $\Psi^{\prime}(x, y)$ parametrically. To compute $v_{n}$ and $w_{n}$ at $y=0$ we solve $\left(62^{\prime}\right)$ and (63') obtaining

$$
w_{n}(x, 0)=a^{-1} v_{n}(x, 0) \text {, }
$$

and

$$
\left\{\begin{array}{l}
v_{0}(x, 0)=\left[h r^{-1}-a^{-1} b \mu Y_{y}^{\prime}\right]^{-1} h r^{2}, \\
v_{1}(x, 0)=\left[h r^{-1}-a^{-1} b u Y_{y}^{\prime}\right]^{-1}\left[b w_{o y}-v_{o y}-h r^{-3}\right], \\
v_{n}(x, 0)=\left[h r^{-1}-a^{-1} b_{\mu} Y_{y}^{\prime}\right]^{-1}\left[b_{\mu}^{n-1} w_{n-1, y}-v_{n-1, y}\right], n \geq 2 .
\end{array}\right.
$$

From these boundary conditions we can successively compute the $v_{n}(x, y)$ using (6) and the $w_{n}(x, y)$ using (8). As a first step we have from $(691)$ and (7)

$$
v_{0}(x, y)=\frac{1-Z}{1+Z}\left(r^{\prime}\right)^{-1}, \quad Z=\frac{\mu b \cos \beta}{a \cos a} .
$$


Proceeding as before we find

$$
w_{0}(x, y)=\frac{2 a^{-1}}{1+Z} \cos \beta \tan a x^{-1 / 2}\left[x+\left(1-\mu^{-2}\right) h \tan ^{3} a\right]^{-1 / 2} \text {. }
$$

Then from $(9),(10)$ and $(70)$ we obtain $v_{1}(x, y)$ in the form

$$
v_{1}(x, y)=\frac{h b w_{o y}-h \nabla_{o y}-h^{-1} \cos ^{3} a}{r^{\prime}(1+Z) \cos ^{2} a}+\left[\frac{1}{2\left(r^{\prime}\right)^{2}}-\frac{\cos a}{2 r^{\prime} h}\right] \text { B } \frac{1-2}{1+Z} \text {. }
$$

The value of $v_{1}(x, 0)$ was obtained from $(691)$ and $B=\frac{1}{\sin \alpha} \frac{\partial}{\partial \alpha}\left(\sin \alpha \frac{\partial}{\partial \alpha}\right)$. We will not compute any more of the $v_{n}$ or $w_{n}$, although they may be obtained in the same way as were $v_{0}, w_{0}$ and $v_{1}$. The terms $v_{0}$ and $w_{0}$ were previously found by E. Gerjuoy $[17]$, by K. O. Friedrichs and J.B. Keller $\left[{ }^{[14]}\right.$ and by others. In the case $\mu<1$ total reflection occurs, and we therefore modify the assumed form of solution (591), (601) by the inclusion of a diffracted wave in the upper medium and an evanescent wave in the lower medium. Thus Instead of (591), (601) we assume

$$
\begin{array}{ll}
u \sim \frac{e^{i k r}}{r}+e^{i k Y} \sum_{n=0}^{\infty}(i k)^{-n_{v}}+e^{i k \varnothing} \sum_{n=0}^{\infty}(i k)^{-n_{s}}, & y>0, \\
u \sim e^{i k^{\prime} I^{\prime}} \sum_{n=0}^{\infty}\left(i k^{\prime}\right)^{-n_{w_{n}}}+e^{i k \cdot \chi} \sum_{n=0}^{\infty}\left(i k^{\prime}\right)^{-n_{t_{n}},} & y<0 .
\end{array}
$$

Froceeding as in the previous example, we find that $v_{0}, w_{0}, Y, Y$ and $v_{1}$ are given by the formlas obtained above and that $\phi$ and $s_{0}$ are also given by the same formulas as and $v_{0}$. The value of $\beta$ is complex for the totally reflected rays, so the evanescent wave decays exponentially. The value of $w_{0}(x, 0)$ for this wave is also given by (681). No other terms in the totally reflected and evanescent waves will be considered.

Now we consider the diffracted wave. Proceeding as in the previous example we insert the expressions for the diffracted and totally reflected waves into the boundary conditions. Upon equating coefficients of powers of 
$k$ we obtain $(78)$ and thus $\varnothing(x, y)$ is given by $(81)$. Thus the diffracted wavefronts are cones with the $y$-axis as axds. In the $x-y$ plane the section of the diffracted wavefront consists of two straight line segments, one of which is shown in Figure 5.

From the other powers of $k$ we find at $y=0$, noting that $y(x, 0)=0$,

$$
s_{n}=a \mu^{-n_{w}} \text {, }
$$

$(801)$

$$
\cos a_{0} s_{n+1}+\frac{\partial s_{n+1}}{\partial y}=+b \mu^{-n} \frac{\partial w_{n}}{\partial y}
$$

Since $w_{0}=0$ at $y=0,\left(79^{\prime}\right)$ shows that $s_{0}=0$ at $y=0$. But then $s_{0}$ vanishes all along each diffracted ray, so we have

$$
s_{0}(x, y)=-0
$$

Then from (6) we find for $s_{1}$

$$
s_{1}(x, y)=s_{1}\left(x-y \tan \alpha_{0}, 0\right) x^{-1 / 2}\left(x-y \tan \alpha_{0}\right)^{1 / 2} \text {. }
$$

Finally after computing $s_{1}(x, 0)$ from $\left(80^{\prime}\right)$ we have

$$
s_{1}(x, y)=-\frac{2 b \sin \alpha_{0}}{a \cos ^{2} \alpha_{0}} x^{-l / 2}\left[x-(y+h) \tan \alpha_{0}\right]^{-3 / 2},\left[x-(y+h) \tan \alpha_{0}\right]>0 .
$$

The leading term in the diffracted wave, $e^{i k \varphi}(i k)^{-1} s_{1}$, was discussed in connection with Example 7, and was also discussed by E. Gerjuoy [17] and by K. O. Friedrichs and J. B. Keller $[14]$, who also obtained it. We will therefore only remark that none of these authors had obtained $v_{1}$, which is of the same order in $k$ as $s_{1}$. For large $x, s_{1}$ behaves like $x^{-2}$ which is a more rapid decay than that of either the incident or the reflected wave. However as $x$ increases these two waves tend to cancel each other, and their sum also ultimately behaves like $x^{-2}$. Therefore the diffracted wave may be comparable to this sum at large horizontal distances from the source. 


\section{General Plane Waves}

By a general plane wave we mean a wave in which the reys are parallel straight lines and the wavefronts therefore parallel planes, but in which the amplitude is not necessarily constant on each plane. For such waves the curvature $G=0$. We will assume that the rays are parallel to the $x$-axis and use $x$ instead of $s$ to denote arclength along them. For such waves equation (3) yields $Y= \pm x+$ const, and as before the constant may be taken as zero without loss of generality. Then $\nabla^{2}=0$, so the solution of (4) is instead of (6)

$$
v_{n}(x, y, z)=v_{n}\left(x_{0}, y, z\right)-\frac{1}{2} \int_{x_{0}}^{x} \nabla^{2} v_{n-1} d x, \quad n \geq 0 .
$$

From (123) we see that $v_{0}$ is independent of $x$. Thus we have

$$
v_{0}(x, y, z)=g_{0}(y, z) \text {. }
$$

Now from (123), (124) find by induction that $v_{n}$ has the form

$$
v_{n}(x, y, z)=\sum_{j=0}^{n} f_{j n}(y, z) x^{j}
$$

Inserting (125) into (123) we obtain the following recursion formulas for the $f_{j n}(y, z)$ with $n \geq 1:$

$$
\begin{array}{ll}
f_{j n}=-\frac{1}{2}\left[j^{-1} \triangle f_{j-1, n-1}+(j+1) f_{j+1, n-1}\right], & 1 \leq j \leq n-2 \\
f_{j n}=-\frac{1}{2} j^{-1} \triangle f_{j-1, n-1}, & j=n-1, n, \\
f_{o n}=v_{n}\left(x_{0}, y, z\right)-\sum_{j=1}^{n} f_{j n} x_{0}^{j}, \quad n \geq 1 . &
\end{array}
$$

In these equations $\Delta$ denotes the two-dimensional Laplacian $\Delta=\frac{\partial^{2}}{\partial y^{2}}+\frac{\partial^{2}}{\partial z^{2}}$, $f_{00}=g_{0}$, and $v_{n}\left(x_{0}, y, z\right)$ is the value of $v_{n}$ on the surface $x=x_{0}(y, z)$. Using the equation (125) for $v_{n}$ in (2) we obtain as the asymptotic expansion of a 
general plane wave

$$
u \sim e^{ \pm i k x} \sum_{n=0}^{\infty}(i k)^{-n} \sum_{j=0}^{n} f_{j n}(y, z) x^{j} .
$$

We will now consider some applications of these results。

Fxample 15. A Line Scurce at the Focus of a Parabolic Cylinder $(u=0)$

Consjder a line source at the focus of a parabolic cylinder (see

Figure 8). The line source creates a cylindrical wave given by (14) or asymptotically by (15), with $m=0$. From the focussing property of the parabola we conclude that this wave will be reflected as a plane wave traveling along the axis of the parabola. Thus this have will have an asymptotic expansion of the form (129) with the $f_{j n}(y)$ satisfying (126), (127) and (128).

To derive these facts in detail let the equation of the parabola be

$$
x=x_{0}(y)=\frac{y^{2}}{4 p}-p
$$

The line source is at the focus of the parabola $(0,0)$, and we denote distance from it by $r$. We assume that the solution $u$ has the expansion

$$
u \sim \frac{e^{i k r}}{\sqrt{i k r}} \sum_{n=0}^{\infty} \rho_{n}(i k r)^{-n}+e^{i k I} \sum_{n=0}^{\infty}(i k)^{-n-(1 / 2)} v_{n} .
$$

On the parabola we assume that $u=0$, and applying this condition to (131) yields

$$
\begin{array}{ll}
I=r, & \text { on } x=x_{0}(y) \\
v_{n}=-p_{n} r^{-n-(1 / 2),} & \text { on } x=x_{0}(y) .
\end{array}
$$

The solution of (3) for $\Psi$ which is outgoing and satisfies (132) can be found, by ray considerations, to be

$$
\mathbf{I}=\mathrm{x}+2 \mathrm{p}
$$

Thus the reflected wave is a general plane wave and $v_{0}$ is of the form (124). Making use of the value of $v_{0}$ on $x=x_{0}(y)$ given by (133) we have 


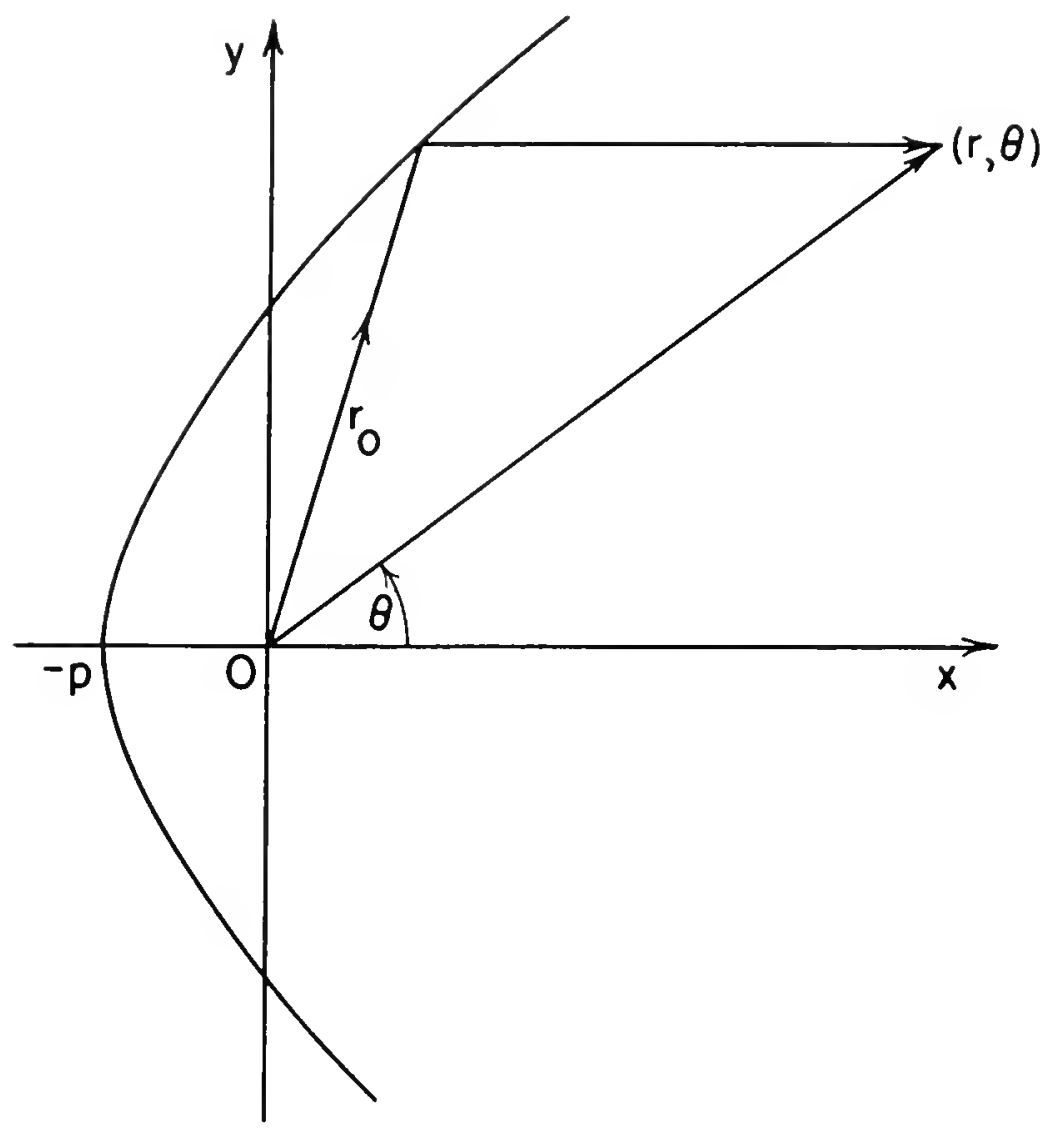

\section{Figure 8}

Reflection from a parabolic cylinder of the cylindrical wave emanating from a line source at the focal line. The focal line is at $(0,0)$, the incident rays are radially directed from it and the reflected rays are parallel to the $x$-axis. The figure also represents the reflection from a paraboloid of revolution of a spherical wave emanating from a point source at the focus. 


$$
v_{0}(x, y)=-\rho_{0}\left(\frac{4 p}{4 p^{2}+y^{2}}\right)^{1 / 2}
$$

We note that $v_{0}$ is independent of $z$, and hence so are all the other $v_{n}$, as was to be expected.

In determining the $v_{n}(x, y)$ for $n \geq I$ it is convenient to introduce two new independent variables $\xi$ and $\eta$ instead of $x$ and $y$. These variables are defined by

$$
\begin{aligned}
& \xi=x+p-\frac{y^{2}}{4 p}, \\
& \eta=p+\frac{y^{2}}{4 p} .
\end{aligned}
$$

In terms of these variables, we find

$$
r^{2}=(\xi+\eta)^{2}-4 p \xi
$$

Equation (123) for the $v_{n}$ now becomes, when (133) is used,

$$
\nabla_{n}(\xi, \eta)=-\rho_{n} \eta^{-[n+(1 / 2)]}-\frac{1}{2 p} \int_{0}^{\xi}\left[(\eta-\rho)\left(D_{\eta}^{2}-2 D_{\xi} D_{\eta}\right)+\eta D_{\xi}^{2}+\frac{1}{2} D_{\eta}-\frac{1}{2} D_{\xi}\right] \nabla_{n-1} d \xi \text {. }
$$

From (135) we find that $v_{0}$ is given by

$$
v_{0}(\xi, \eta)=-\eta^{-1 / 2} \text {. }
$$

Now using $(140)$ in (139) we find that $v_{n}$ is given by

$$
v_{n}(\xi, \eta)=\sum_{l=0}^{n} \sum_{m=0}^{n} a_{l m, n+1} \xi^{l} \eta^{-[l+m-(1 / 2)]_{p}-n+m}
$$

The constants a lmn $_{\text {are determined by the recursion formulas }}$

$$
a_{\text {omn }}=0, \quad m \neq n,
$$

$$
a_{\text {onn }}=-\rho_{n} \text {, }
$$

(144) $a_{\ell m, n+1}=-\frac{\ell+1}{2} a_{\ell+1, m, n}+\left(\ell+m-\frac{3}{2}\right)\left(\ell+m-\frac{1}{2}\right)(2 \ell)^{-1} a_{\ell-1, m-1, n}$

$$
+\left(\ell+m-\frac{1}{2}\right) a_{\ell, m-1, n}-\left(\ell+m+\frac{1}{4}\right) a_{l m n}-\left(\ell+m-\frac{1}{2}\right)(\ell+m)(2 \ell)^{-1} a l-1, m, n, \quad \ell \neq 0 .
$$


We finally have from (131), (134) and (141) the following asymptotic expansion for u:

(145) $u(\xi, \eta) \sim \frac{e^{i k r}}{\sqrt{i k r}} \sum_{n=0}^{\infty} \rho_{n}(i k r)^{-n}+\frac{e^{i k(2 p+x)}}{\sqrt{i k p}} \sum_{n=0}^{\infty}(i k p)^{-n} \sum_{\substack{l=0 \\ l=0}}^{l=n} a_{l m n}\left(\xi p^{-1}\right)^{l}\left(\eta^{-1} p\right)^{\ell+m+\frac{1}{2}}$. The first few terms in $(1 / 45)$ yield

$$
\text { (146) } \begin{aligned}
u(\xi, \eta) \sim & \frac{e^{i k r}}{\sqrt{i k r}} \sum_{n=0}^{\infty} \rho_{n}(i k r)^{-n}-\frac{e^{i k(2 p+x)}}{\sqrt{i k p}} \cdot \\
& \left\{1+\frac{1}{8 i k p}\left[\left(p \eta^{-1}\right)^{3 / 2}-2 \xi \eta^{-3 / 2} p^{1 / 2}+3 \xi \eta^{-5 / 2} p^{3 / 2}\right]+\cdots\right\}
\end{aligned}
$$

Example 16. A Line Source at the Focus of a Farabolic Cylinder $\left(\frac{\partial u}{\partial v}=0\right)$

If replace the boundary condition $u=0$ of the previous problem by the condition $\frac{\partial u}{\partial v}=0$, we obtain a different problem which can be treated in essentially the same way. The first difference between the two problems appears in the expression for $v_{n}$ on the boundary, which is given by the following equations instead of (133):

$$
\begin{array}{ll}
v_{0}=+\rho_{0} r^{-1 / 2}=\eta^{-1 / 2}, & \text { on } x=x_{0}(y), \\
v_{n}=-\left(\frac{\partial \mathbf{I}}{\partial v}\right)^{-1}\left[\frac{\partial}{\partial v}\left(\rho_{n-1} r^{-1 / 2}+v_{n-1}\right)+\rho_{n} r^{-1 / 2} \frac{\partial r}{\partial v}\right], \quad \text { on } x=x_{0}(y), \\
v_{n}=p^{-1}\left[(\eta-p) D_{\eta}-\eta D_{\xi}\right]\left[\rho_{n-1} r^{-1 / 2} v_{n-1}\right]+\rho_{n} \eta-[n+(1 / 2)], \text { on } \xi=0 .
\end{array}
$$

The result ( $1 / 1 \mathcal{I})$ is again obtained for $v_{n}(\xi, \eta)$ and $a_{/ m n}$ is again given by (1144) for $l \neq 0$, while for $l=0$ we have instead of (142), (143) the following: 
(149)

$$
a_{0, n+1, n+1}=\rho_{n}
$$

$$
a_{0, m, n+1}=\left(m-\frac{3}{2}\right) a_{0, m-1, n+1}-\left(m-\frac{1}{2}\right) a_{0 m n}-a_{1 m n} \quad m \neq n+1 \text {. }
$$

The expansion of $u$ is given by (145) with the appropriate a/mn . The first few terms in this expansion yield

$$
\begin{aligned}
u(\xi, \eta) & \sim \frac{e^{i k r}}{\sqrt{i k r}} \sum_{n=0}^{\infty} \rho_{n}(i k r)^{-n}+\frac{e^{i k(2 p+x)}}{\sqrt{i k \eta}} \cdot \\
& \left\{1+\frac{1}{8 i k p}\left[\left(p \eta^{-1}\right)^{3 / 2}-2 \xi \eta^{-3 / 2} p^{1 / 2}+3 \xi \eta^{-5 / 2} p^{3 / 2}+4 \eta^{-1 / 2} p^{1 / 2}\right]+\cdots\right\} .
\end{aligned}
$$

Example 17. A Point Source at the Focus of a Paraboloid of Revolution $(u=0)$ We will now determine the field produced by a point source located at the focus of a parabolold of revolution with $u=0$ on the paraboloid. As in the two preceding examples, we expect the reflected field to be a general plane wave, and this is indeed the case. The wave produced by a point source is spherical and independent of $\theta$ and $\varnothing$, so we may describe it by (100) or (101) with $m=0$. We will use (101) with $A=k$, so the spherical wave becomes $R^{-1} e^{1 k R}$, which is the customary form. Then we assume that

$$
u \sim \frac{e^{i k R}}{R}+e^{i k \Psi} \sum_{n=0}^{\infty}(i k)^{-n_{v_{n}}} \text {. }
$$

Here $R$ denotes distance from the focus located at $(p, 0,0)$ on the $x$-axis, which is the axis of the paraboloid. We write the equation of the parabolcid as 
$-50-$

$$
x_{0}=x_{0}(y, z)=\frac{r^{2}}{4 p}-p
$$

Here $r=\left(x^{2}+y^{2}\right)^{1 / 2}$ is radial distance from the $x$-axis.

Upon inserting (152) into the boundary condition $u=0$ and equating

coefficients of powers of $k$, we obtain on the paraboloid

$$
\begin{aligned}
& I=R, \\
& \nabla_{0}=-R^{-1}, \\
& \nabla_{n}=0, \quad n \geq 1 .
\end{aligned}
$$

As in the preceding problems, we find that $I$ is given by (157)

$$
I=x+2 p
$$

From (155) and (12/1) $v_{0}$ is found to be

$$
v_{0}=-\left(\frac{4 p}{4 p^{2}+r^{2}}\right)^{1 / 2}
$$

From (158) and (125) it is clear that all the $v_{n}$ will be independent of the angular coordinate $\theta$ in the $y, z$ plane, as was to be expected.

To determine the $v_{n}$ for $n \geq I$ it is again convenient to replace $x$ and $\mathrm{y}$ by the two new independent variables $\xi, \eta$ defined by

$$
\begin{aligned}
& \xi=x+p-\frac{r^{2}}{4 p} \\
& \eta=p+\frac{r^{2}}{4 p} .
\end{aligned}
$$

We then have

$$
R^{2}=(\xi+\eta)^{2}-4 p \xi
$$

E guation (123) for the $v_{n}$ now becomes, when use is made of (150),

(262)

$$
v_{n}(\xi, \eta)=-\frac{1}{2 p} \int_{0}^{\xi}\left[(\eta-p)\left(D^{2}-2 D_{\xi} D\right)+\eta D_{\xi}^{2}+D_{\eta}-D_{\xi}\right] v_{n-1} d \xi \text {. }
$$

From (158) we see that $v_{0}$ is given by 


$$
v_{0}(\xi, \eta)=-\eta^{-1}
$$

Now beginning with this value for $v_{0}$, and determining the $v_{n}$ successively from (162) we find

$$
v_{n}(\xi, \eta)=\sum_{l=0}^{n} \sum_{m=0}^{n} a_{\ell m n} \xi^{l} \eta^{-m-\ell-1} p^{m-n}
$$

The constants $a_{\ell m n}$ are given by the formulas

$$
\begin{aligned}
& a_{\infty}=-1 \text {, } \\
& a_{\text {onn }}=0, \quad n \geq 1, \\
& a_{o m, n+1}=-(m+1) a_{o m n}+m a_{o, m-1, n}-a_{1 m n}, \quad 0 \leq m \leq n-1, \\
& a_{\ell m, n+1}=-(m+l)^{2}(2 l)^{-1} a_{l-1, m n}+(m+l)_{l, m-1, n^{-}} \frac{\ell+1}{2} a_{\ell+1, m n} \\
& \left.-\left(\mathrm{m}+\ell+\frac{1}{2}\right) a_{\ell m n}+(m+\ell-1)(m+l)(1 / 2) a\right) \ell-1, m-1, n, \quad \ell>0 .
\end{aligned}
$$

Finally, from (152), (157) and (164) we have the following asymptotic expansion for u:

$$
u(\xi, \eta) \sim \frac{e^{i k R}}{R}+\frac{e^{i k(2 p+x)}}{\eta} \sum_{n=0}^{\infty}(i k p)^{-n} \sum_{\ell=0}^{n} \sum_{m=0}^{n}{ }^{a}{ }_{l m n} \xi^{\ell} \eta^{-m-l_{p} m} .
$$

The first few terms in this expansion are

$$
u(\xi, \eta) \sim \frac{e^{i k R}}{R}-\frac{e^{i k(2 p+x)}}{\eta}\left\{1-\frac{\xi}{i k \eta}\left[(2 p)^{-1}-\eta^{-1}\right]+\cdots\right\} \text {. }
$$

The present problem is similar to one treated by F. G. Friedlander [79] who considered an incident spherical pulse instead of an incident spherical time periodic wave. He obtained a series solution of the problem, the successive terms of which contain the discontinuities in the successive time derivatives of the reflected pulse. Consequently as Luneburg [1] and Kline [2] have shown, the terms in his solution are related to corresponding terms in the asymptotic expansion with respect to $k$ of the solution of the time periodic problem. There- 
fore our recursion formulas are similar to his. In fact the variables $\xi$ and $\eta$, which simplify the calculations considerably, were introduced by him.

Example 18. A Point Source at the Focus of a Paraboloid of Revolution $\left(\frac{\partial u}{\partial v}=0\right)$ We now reconsider the preceding problem but with the boundary condition $\frac{\partial u}{\partial \nu}=0$ on the paraboloid. Froceeding as before from (152) we again obtain (153), (154) and (157), but (155) and (158) occur with the minus signs replaced by plus signs. Instead of (156) we find

$$
v_{n}=-\left(\frac{\partial \Psi}{\partial v}\right)^{-1} \frac{\partial v_{n-1}}{\partial v}=p^{-1}\left[(\eta-p) D_{\eta}-\eta D_{\xi}\right] v_{n-1} \text {, at } \xi=0 .
$$

The second expression on the right in (171) results when the coordinates $\xi$ and $\eta$ are introduced. The expression (162) for $v_{n}(\xi, \eta)$ must be modified by the addition on the right side of the right member of (171). Then we again obtain (164) for the $v_{n}(\xi, \eta)$ where the ${ }^{a} \ell_{m n}$ satisfy exactly the same recursion formulas as in the previous example, except that

$$
a_{000}=+1
$$

The asymptotic expansion of $u(\xi, \eta)$ is given by (169) with the appropriate

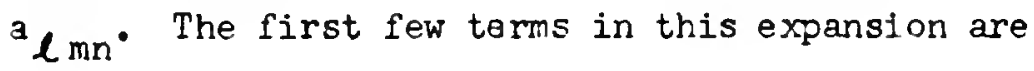

$$
u(\xi, \eta) \sim \frac{e^{i k R}}{R}+\frac{e^{i k(2 p+x)}}{\eta}\left\{1-\frac{1}{i k p}\left[1+\frac{\xi}{2 \eta}+\frac{\xi p}{\eta^{2}}\right]+\cdots\right\} .
$$

7. Arbitrary Waves

In Section 2 we showed how to obtain the asymptotic expansion of a solution of (1) in the form (2). The construction of such an asymptotic expansion first requires the determination of a phase function satisfying the efconal equation (3) and then the determination of the associated system 
of rays. These rays are the orthogonal trajectories of the waverronts (i.e., the surfaces $Y=$ constant) and they are straight lines. Alternatively we may begin with a normal congruence of rays and then construct the associated phase function $\mathbf{Y}$. Once the rays are known, the coefficients $\nabla_{n}$ can be computed successively using (6), merely by integration along the rays. In the subsequent sections this construction was carried out for cylindrical, general cylindrical, general spherical and general plane waves, in which the wavefronts are families of concentric circular cylinders for the first two cases, and for the other two cases, concentric spheres and parallel planes respectively. Now we will show how to carry out the construction for an arbitrary wave, i.e., one in which one of the wavefronts is an arbitrary surface.

Let us begin by selecting an arbitrary surface $Q$ as the wavefront $\bar{I}=0$. We introduce the surface coordinates $x_{2}, x_{3}$ on $Q$ by means of the two families of lines of curvature of $Q$. On each line of the first family, $x_{2}$ has a constant value and $x_{3}$ denotes arclength along the line from a fixed line of the second. family. Similarly on each line of the second family, $x_{3}$ has a constant value and $x_{2}$ denotes arclength along the line from a fixed line of the first family. Now we introduce the normals to $Q$ and the distance $s$ from $Q$ along a normal, measured positively on one side and negatively on the other. Then $s, x_{2}, x_{3}$ are three orthogonal coordinates which uniquely locate a point, although a single point may have more than one set of coordinates if it liee on more than one normal to $Q$.

We can now construct a solution $I$ of (3) which is equal to zero on Q. There are exactly two such solutions, namely $\bar{I}= \pm \mathrm{s}$, and we will consider the solution $I=+s$. The wavefronts $\Psi=$ constant are the surface $Q$ and the surfaces parallel to $1 t$, while the rays are the normals to $Q$, on each of which $x_{2}$ and $x_{3}$ are constant. 
In order to apnly (6) for the determination of the $v_{n}$, we must calculate the Laplacian operator in $\approx u r$ orthogonal coordinate system. To this end introrluce the principal radii of curvature $p_{2}\left(x_{2}, x_{3}\right)$ and $p_{3}\left(x_{2}, x_{3}\right)$ of the surface $Q$ at the point $x_{2}, x_{3}$. In terms of these radii the metric coefficients in this coordinate system are

$$
h_{1}=1, h_{2}\left(s, x_{2}, x_{3}\right)=\frac{\rho_{2}\left(x_{c}, x_{3}\right)+s}{\rho_{2}\left(x_{2}, x_{3}\right)}, \quad h_{3}\left(s, x_{2}, x_{3}\right)=\frac{\rho_{3}\left(x_{2}, x_{3}\right)+s}{\rho_{3}\left(x_{2}, x_{3}\right)} .
$$

Then we have

$$
\begin{aligned}
\nabla^{2}=\frac{\partial^{2}}{\partial s^{2}} & +\frac{1}{\rho_{2}+s} \frac{\partial}{\partial s}+\frac{1}{\rho_{3}+s} \frac{\partial}{\partial s} \\
& +\left(\frac{\rho_{2}}{\rho_{2}+s}\right)^{2}\left(\frac{\partial^{2}}{\partial x_{2}^{2}}-\frac{s}{\rho_{3}\left(\rho_{3}+s\right)} \frac{\partial \rho_{3}}{\partial x_{2}} \frac{\partial}{\partial x_{2}}+\frac{s}{\rho_{2}\left(\rho_{2}+s\right)} \frac{\partial \rho_{2}}{\partial x_{2}} \frac{\partial}{\partial x_{2}}\right) \\
& +\left(\frac{\rho_{3}}{\rho_{3}+s}\right)^{2}\left(\frac{\partial^{2}}{\partial x_{3}^{2}}-\frac{s}{\rho_{2}\left(\rho_{2}+s\right)} \frac{\partial \rho_{2}}{\partial x_{3}} \frac{\partial}{\partial x_{3}}+\frac{s}{\rho_{3}\left(\rho_{3}+s\right)} \frac{\partial \rho_{3}}{\partial x_{3}} \frac{\partial}{\partial x_{3}}\right) .
\end{aligned}
$$

Furthermore the Gaussian curvature of the surface $I$ = $s$ - constant is

$$
G\left(s, x_{2}, x_{3}\right)=\frac{1}{\left(\rho_{2}+s\right)\left(\rho_{3}+s\right)}
$$

Thus (6) becomes

$$
\begin{aligned}
v_{n}\left(s, x_{2}, x_{3}\right)= & v_{n}\left(s_{0}\left[x_{2}, x_{3}\right], x_{2}, x_{3}\right)\left[\frac{\left(\rho_{2}+s_{0}\right)\left(\rho_{3}+s_{0}\right)}{\left(\rho_{2}+s\right)\left(\rho_{3}+s\right)}\right]^{1 / 2} \\
& -\frac{1}{2\left(\rho_{2}+s\right)^{1 / 2}\left(\rho_{3}+s\right)^{1 / 2}} \int_{s}^{s}\left(\rho_{2}+\tau\right)^{1 / 2}\left(\rho_{3}+\tau\right)^{1 / 2} \nabla^{2} v_{n-1} d \tau .
\end{aligned}
$$

In (177), $v_{n}\left(s_{0}\left[x_{2}, x_{3}\right], x_{2}, x_{3}\right)$ denotes the value of $v_{n}$ at the point $s_{0}$ on the ray $x_{2}=$ constant, $x_{3}=$ constant. For example, if $s_{0}=0$, then $\nabla_{n}\left(s_{0}, x_{2}, x_{3}\right)$ denotes the value of $v_{n}$ at the point $x_{2}, x_{3}$ on $Q$. 
When $\mathrm{n}=0$ the integral in (177) is absent, so the first term alone yields $v_{0}$. Its dependence upon $s$ is particularly simple, and we note that as $s$ becomes large, $v_{0}$ decreases like $s^{-1}$ provided that both $\rho_{2}$ and $\rho_{3}$ are finite. If either $\rho_{2}$ or $\rho_{3}$ is infinite while the other is finite, $v_{0}$ decreases like $|s|^{-1 / 2}$ for large $s$, and if both are infinite $v_{0}$ is independent of $s$. In addition we note that $v_{0}$ becomes infinite at the two points $s=-p_{2}, s=-p_{3}$ on each ray, or at one or no points if either or both radil are infinite. The locus of points at which $v_{0}$ becomes infinite is the caustic surface of the ray system, and it has two branches which touch on rays for which $\rho_{2}=\rho_{3}$. From the fact that $v_{0}$ becomes infinite on the caustic, we conclude that the asymptotic expansion (2) is not valid on the caustic. This is indeed the case, for it has been shown by I. Kay and J. B. Keller [16] that on a caustic $u$ is asymptotic to a positive fractional power of $k$. The expansion of $u$ on and near the caustic can be found from the expansion (2) by the method of [16].

We have thus seen that the behavior of $v_{0}$ on the ray through the point $x_{2}, x_{3}$ on $Q$ depends solely on the radii of curvature of $Q$ at $x_{2}, x_{3}$. If the point is elliptic, there are two caustic points on the ray at the two centers of principal curvature of $Q$, which lie on the same side of $Q$, and $v_{0}$ decreases as in a spherical wave for large $s$. If the point is umbilical, the two caustic points coincide, while if it is hyperbolic they lie on opposite sides of $Q$. If the point is cylindrical there is only one caustic point on the ray and $v_{0}$ varies as in a cylindrical wave for all $s$. If the point is planar there are no caustic points on the ray and $v_{0}$ is constant along it, just as in a plane wave. We will now see that the nature of the point $x_{2}, x_{3}$ on $Q$ also determines the behavior of the other $v_{n}$.

By examining the expression for $\nabla^{2}$ in (175) and the form of the second term in (177), we see that for large $s$ this term is $O\left(s^{-1}\left[1+v_{n-1}\right]\right)$ provided 
$\rho_{2}$ and $\rho_{3}$ are finite. This follows because in this case $\nabla^{2}$ reduces the order of $v_{n-1}$ with regard to $s$ by two, while the factors in the integrand of (177) and the integration increase the order by two, and the factors outside the integral redice the order by one. Thus all the $v_{n}$ tend to zero as $s$ becomes infinite. In the same way we see that the second term in $(177)$ is $O\left(s_{n-1}\right)$ if $\rho_{2}$ is infinite and

$$
\frac{\partial^{2} v_{n-1}}{\partial x_{2}^{2}}-\frac{s}{\rho_{3}\left(\rho_{3}+s\right)} \frac{\partial \rho_{3}}{\partial x_{2}} \frac{\partial v_{n-1}}{\partial x_{2}} \neq 0
$$

or if $\rho_{3}$ is infinite and

$$
\frac{\partial^{2} v_{n-1}}{\partial x_{3}^{2}}-\frac{s}{\rho_{2}\left(\rho_{2}+s\right)} \frac{\partial \rho_{2}}{\partial x_{3}} \frac{\partial v_{n-1}}{\partial x_{3}} \neq 0
$$

or if $\rho_{2}$ and $\rho_{3}$ are both infinite and

$$
\frac{\partial^{2} v_{n-1}}{\partial x_{2}^{2}}-\frac{s}{\rho_{3}\left(\rho_{3}+s\right)} \frac{\partial \rho_{3}}{\partial x_{2}} \frac{\partial v_{n-1}}{\partial x_{2}}+\frac{\partial^{2} v_{n-1}}{\partial x_{3}^{2}}-\frac{\varepsilon}{\rho_{2}\left(\rho_{2}+s\right)} \frac{\partial \rho_{2}}{\partial x_{3}} \frac{\partial v_{n-1}}{\partial x_{3}} \neq 0 .
$$

Thus in any one of these cases $v_{n}$ decreases more slowly than $v_{n-1}$ as s increases, and in general $v_{n}$ will in fact increase, becoming infinite with $s$. Furthermore the greater $\mathrm{n}$ is, the more rapidly will $\mathrm{v}_{\mathrm{n}}$ become infinite.

We thus find that on a ray through a planar or cylindrical point of $Q$ the $v_{n}$ for $n \geq 1$ will in general become infinite $2 s$ s does, groving like $s^{n-(1 / 2)}$ (or perhaps with some factors s replaced by log $s$ ) for a cylindrical point and like $s^{n}$ for a planar point. From this result we conclude that in general on rays through planar or cylindrical points of a the asymptotic $\in \mathrm{x}-$ pansion (2) is non-uniform. These results have been exemplified by equations (89) and (126) for general cylindrical and general plane waves. For ordinary cylindrical or plane waves, un the other hand, although one or two radil are infinite, the other quantities appearing in the conditions above are zero. 
In such cases the second term in (177) is $O\left(s^{-1} v_{n-1}\right)$ and the $v_{n}$ all tend to zero as $s$ becomes infinite.

By means of the preceding results we can treat the problem of reflection of any incident field from a surface $S$ of arbitrary shape. Let us suppose that on $S$ either $u=0$ or $\frac{\partial u}{\partial v}=0$. We begin by expanding the given incident field asymptotically and determining the asscciated ray system. Then we determine the reflected ray system produced by reflection of the incident rays from $S$. Next we write the field at any point $P$ as a sum of terms, one term for each ray through the point P. Each of these terms is an asymptotic expension of the type treated in section 2. The phase for each term is determined from the phase appropriate to the incident ray at the reflection point and the length of the reflected ray to the point $P$. The value of each $v_{n}$ on $S$ is obtained from the boundary condition in which the asymptotic expansion of the incident wave appears. The value of $v_{n}$ of $f$ $S$ is computed from (177) as indicated above.

The method just described is the one which has been employed in the preceding sections. It has been applied to the reflection (and transmission) of a spherical wave at an arbitrary surface by J. B. Keller and H. B. Keller ${ }^{[22]}$ and to the reflection (and transmission) of an arbitrary wave at an arbitrary surface by J. B. Keller and S. Preiser [23]. However, in both of these cases only $v_{0}$, the leading term in the reflected (and transmitted) fleld, was determined. 
In the next two sections we will apply the considerations of this section to two general classes of waves. In Section 8 we consider two dimensional or cylindrical waves, i.e., waves independent of one Cartesian coordinate. The result enables us to treat reflection of any two dimeneional or cylindrical wave from a two dimensional or cylindrical reflector. As an example we treat reflection of a plane wave from a circular cylinder. In Section 9 we consider three dimensional waves possessing an axis of symmetry. In this case the wavefronts are surfaces of revolution. This result enables us to treat reflection from any surface of revolution. Of course the incident wave must also be synimetric about the same axis as the body. The result is exemplified by the treatment of reflection of a plane wave from a sphere.

8. Two dimensional waves

Let us consider a two dimensional wave, i.e., one which is independent of the Cartesian coordinate $z$. Then the wavefronts $\mathbf{I}=$ constant are cylinders perpendicular to the $x y$ plane. The intersection of a wavefront with this plane is a curve which we will also call a wavefront. The fanily of wavefronts (In the plane) is a family of parallel curves, the common normals to which are the rays. These rays will in general possess an envelope which is called the caustic of the family of wavefronts (or of each wavefront). We introduce the distance $s$ measured along a ray from the caustic as one coordinate and the angle $\beta$ between a ray and the positive $x$-axis as the other coordinate (see Figure 9a). Although these coordinates are not orthogonal they seem to be more appropriate than the coordinates of the preceding section.

We suppose that the equation of the caustic is given in terms of the parameter $\beta$ by the equations $x=\xi(\beta), y=\eta(\beta)$. Then for any point $x, y$ we have the transformation equations 


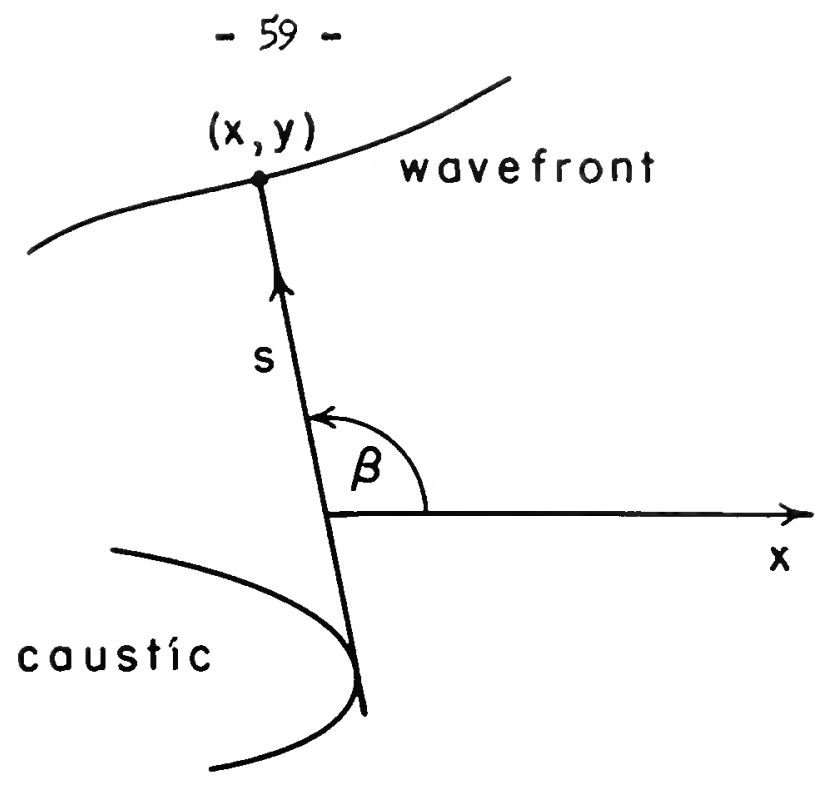

Fig. 9a

Caustic coordinates. A point $(x, y)$ is represented by the caustic coordinates $(s, \beta)$. $s$ is the distance from the point to the caustic, measured along the ray through the point. The angle $\beta$ is the angle between the ray and the positive $x-2 x i s$.

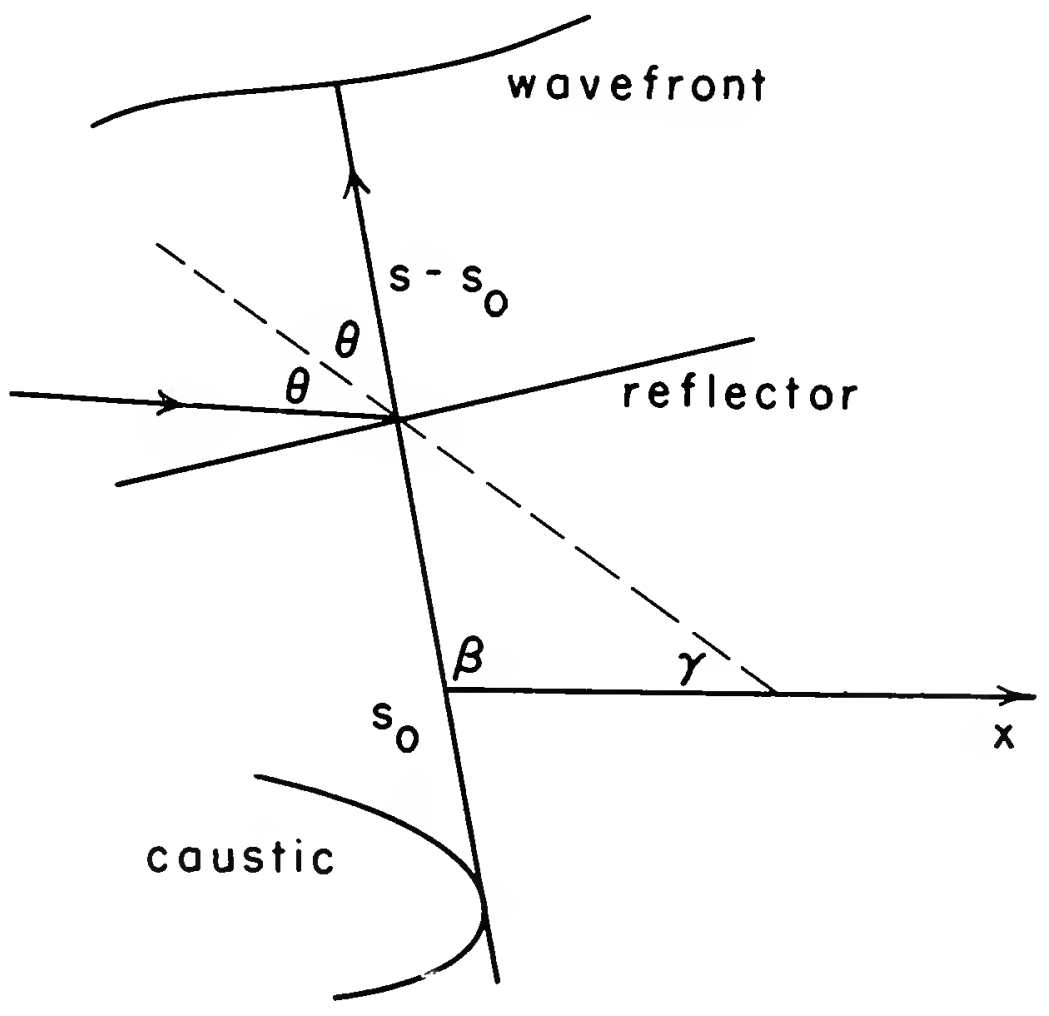

Fige $9 \mathrm{~b}$

A ray incident on the reflector at angle $\theta$ yields a reflected ray with angle of reflection $\theta$. The reflected ray makes the angle $\beta$ with the positive $x$-axis. The normal to the reflector at the point of reflection makes the angle $\gamma$ with the negative $x$-axis. The point of reflection is at distance $s_{0}$ from the caustic, measured along the reflected ray. 
$-60-$

(178)

$$
x=\xi(\beta)+s \cos \beta, \quad y=\eta(\beta)+s \sin \beta .
$$

By making use of (178) we find for the Laplacian operator in the new coordinates

$$
\nabla^{2}=\left(1+\frac{a}{s^{2}}\right) \frac{\partial^{2}}{\partial s^{2}}+\left(\frac{1}{s}-\frac{c}{s^{2}}-\frac{a}{s^{3}}\right) \frac{\partial}{\partial s}-\frac{2 b}{s^{2}} \frac{\partial^{2}}{\partial s \partial \beta}+\frac{1}{s^{2}} \frac{\partial^{2}}{\partial \beta^{2}}+\frac{b}{s^{3}} \frac{\partial}{\partial \beta}
$$

where

(180) $\quad a=\dot{\xi}^{2}+\dot{\eta}^{2}, \quad b=\dot{\xi} \cos \beta+\dot{\eta} \sin \beta, \quad c=\ddot{\xi} \cos \beta+\ddot{\eta} \sin \beta$.

In (180) the dot denotes differentiation with respect to $\beta$. The quantities $a, b$ and $c$ have the following interpretation. If $\vec{s}(\beta)$ denotes the distance along the ray $\beta$ from the caustic to a wavefront, then

$$
a=\left(\bar{s}_{\beta}\right)^{2}=b^{2}, \quad b=-\bar{s}_{\beta}, \quad c=-\bar{s}_{\beta \beta}=b_{\beta} \text { 。 }
$$

Since $\overline{\mathbf{s}}(\beta)$ also denotes the radius of curvature of the wavefront, we see that $\bar{s}(\beta)$ is constant if and only if the wavefront is a circle. Thus for a family of circular wavefront $a=b=c=0$.

In terms of the present coordinates (6) takes the form

$$
v_{n}(s, \beta)=v_{n}\left(s_{0}, \beta\right)\left[\frac{s}{8}\right]^{1 / 2}-\frac{1}{2 s^{1 / 2}} \int_{s_{0}}^{s} \tau^{1 / 2} \nabla^{2} v_{n-1}(\tau, \beta) d \tau \text {. }
$$

One may show by induction, using (179) and (182), that the $\nabla_{n}$ are given by (183)

$$
v_{n}(s, \beta)=\sum_{j=0}^{3 n} f_{j n}(\beta) s^{-1 / 2-j} \text {. }
$$

The coefficient $f_{j n}$ are given recursively by

$$
\left.\begin{array}{rl}
f_{j n}=\frac{1}{2 j}\left\{\left(j-\frac{1}{2}\right)^{2} f_{j-1, n-1}+\ddot{f}_{j-1, n-1}\right. & +2 b(j-1) \dot{f}_{j-2, n-1}+c\left(j-\frac{3}{2}\right) f_{j-2, n-1} \\
& +a\left(j-\frac{5}{2}\right)\left(j-\frac{1}{2}\right) f_{j-3, n-1}
\end{array}\right\} \begin{aligned}
& j \neq 0
\end{aligned}
$$

(184)

$$
\begin{aligned}
& f_{o n}=v_{n}\left(s_{0}, \beta\right) s_{0}^{1 / 2}-\sum_{j=1}^{3 n} f_{j n} s_{0}^{-j} \quad n \neq 0 \\
& f_{o 0}=v_{0}\left(s_{0}, \beta\right) s_{0}^{1 / 2} .
\end{aligned}
$$


In (184) we use the convention $f_{j n}=0$ if $j<0$ or $j>3 n$.

These results, (183) and (184), given the coefficients in the expansion of any two dimensional wave. We may use these results to treat reflection of an arbitrary cylindrical wave from an arbitrary cylindrical reflector. As in the previous problems, we may choose $s=s_{0}(\beta)$ to be the equation of the reflector surface (see Figure $9 b$ ). Then the $\nabla_{n}\left(s_{0}, \beta\right)$ are determined by the incident field and the boundary conditions on the reflector, so all the $v_{n}$ can be computed.

In the application to reflection the equation of the reflector is usually given in the form $y=g(x)$ and the phase of the incident wave in the form $Y=I(x, y)$. It is therefore convenient to express $a, b$, and $c$ in terms of these given functions. To this end it is convenient to introduce the angle of incldence $\theta$ given by

$$
\cos \theta=\frac{I_{x}[x, g(x)] g^{\prime}-I_{y}[x, g(x)]}{\sqrt{1+\left(g^{\prime}\right)^{2}}} .
$$

It is also useful to define the angle $\varphi$ between the normal to the reflector and the $x$-axis. Then the angle $\beta$ between the reflected ray and the $x$-axis is

$$
\beta=\pi-\theta-\gamma \text {. }
$$

Now if $\rho$ denotes the radius of curvature of the reflector we find that $s_{0}$, the distance along the reflected ray from the reflector to the caustic is

$$
s_{0}=\frac{\rho}{2} \cos \theta \text {. }
$$

The radius of curvature $\rho$ and the derivative of $\varphi$ are easily seen to be given by

$$
\begin{aligned}
& \rho=\left[1+\left(g^{\prime}\right)^{2}\right]^{3 / 2}\left|g^{n}\right|^{-1} \\
& \gamma^{\prime}=-g^{\prime \prime}\left[1+\left(g^{\prime}\right)^{2}\right]^{-1} .
\end{aligned}
$$

Since the equation of a reflected wavefront is $Y+s-s_{0}=$ constant, we have from (181) 


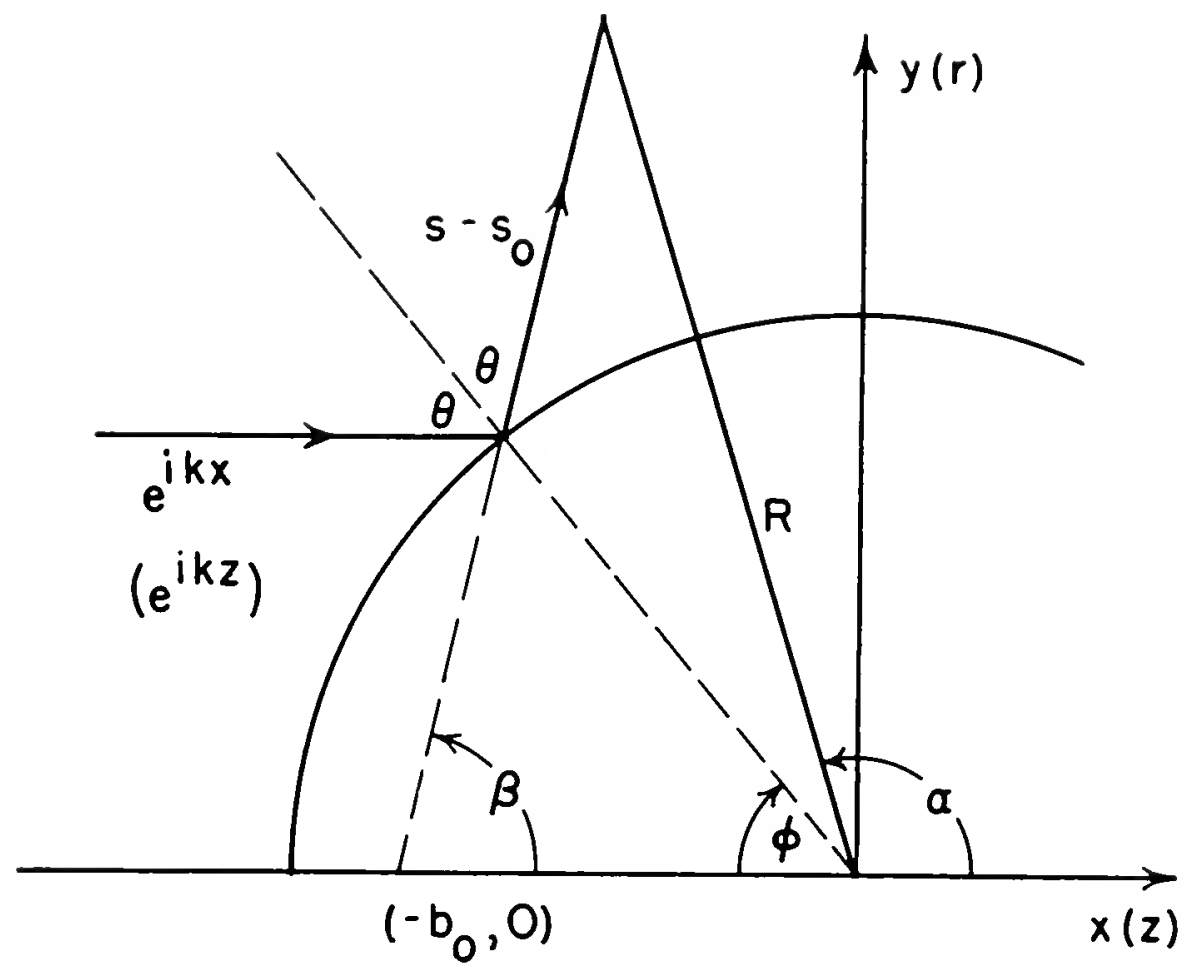

Fig. 10

Reflection of a plane wave incident from the left on a circular cylinder or on a sphere of radius $b$. In the first case, the rectangular coordinates $(x, y)$ of a point are to be related to $(s, \beta)$ and in the latter case, the cylindrical coordinates $r, z$ are to be related to $s, \beta$. 
(190)

$$
\begin{aligned}
& b=Y_{\beta}-s_{O \beta}=\frac{1}{\frac{d \beta}{d x}} \frac{d}{d x}\left(1-s_{0}\right)=\frac{1}{\theta^{\prime}+\gamma^{\prime}}\left\{\frac{\left[1+\left(g^{\prime}\right)^{2}\right]\left[I_{x^{\prime}}-I_{y}\right]}{2\left|g^{\prime \prime}\right|}-Y^{\prime}\right. \\
& a=b^{2}, \quad c=-\frac{1}{\theta^{\prime}+\gamma^{\prime}} b^{\prime} \cdot
\end{aligned}
$$

It should be noted that if the parameter $x$ is used instead of $\beta$ in (184) then the $\beta$ derivatives must be converted to $x$-derivatives.

As an example of (190), consider an incident plane wave for which $\mathbf{Y}=\mathbf{x}$. Then (190) becomes

$$
b=\frac{1}{2 \theta^{\prime}}\left\{\frac{y^{\prime}}{2\left|\theta^{\prime}\right|}-x\right\}^{\prime}, \quad a=b^{2}, \quad c=-\frac{1}{2 \theta^{\prime}} b^{\prime} .
$$

In case the equation is given parametrically as $x(t), y(t)$, then (191) applies with the $x$-derivatives replaced by $t$ derivatives. If $t$ denotes arclength then $\theta_{t}$ is just the curvature of the reflector.

Example 19: Diffraction of a plane wave by a circular cylinder $(u=0)$

Consider the plane wave $e^{i k x}$ incident from the left on a circular cylinder whose equation is (see Figure 10)

$$
\left\{\begin{array}{l}
x=-b_{0} \cos \phi=-b_{0} \sin \beta / 2 \\
y=b_{0} \sin \phi=b_{0} \cos \beta / 2 .
\end{array}\right.
$$

We will calculate the asymptotic expansion of the reflected field. The field associated with the diffracted rays will not be computed since its order is higher than that of the complete asymptotic expansion of the reflected field. Upon applying the boundary condition $u=0$ to the incident plus reflected fields we find

$$
\begin{aligned}
& Y\left(s_{0}, \beta\right)=x=-b_{0} \sin \beta / 2 \\
& v_{0}\left(s_{0}, \beta\right)=-1, \quad v_{n}\left(s_{0}, \beta\right)=0 \quad \text { for } n \geq 1 .
\end{aligned}
$$


From (193) and (187), one finds that the phase of the reflected field is given by $\mathbf{Y}(s, \beta)=\mathbf{Y}\left(s_{0}, \beta\right)+\mathbf{s - s _ { 0 }}=s-\frac{3}{2} b_{0} \sin \beta / 2$

To use the recursion relations (184), we must express a, b and $c$ in terms of $\beta$. From (191) and (192) we find

$$
\begin{gathered}
a=\frac{9}{16} b_{0}^{2} \cos ^{2} \beta / 2, \quad b=-\frac{3}{4} b_{0} \sin \phi=-\frac{3}{4} b_{0} \cos \beta / 2 \\
c=\frac{3}{8} b_{0} \cos \phi=\frac{3}{8} b_{0} \sin \beta / 2 .
\end{gathered}
$$

Using these coefficients in (184) we find, and verify by induction, that the $\mathrm{f}_{\text {jn }}$ have the form

$$
f_{j n}=2^{-(4 n+j+3 / 2)} b_{0}^{j-n+1 / 2} \sum_{t=0}^{n} a_{j t n}(\sin \beta / 2)^{j-2 t-n+\frac{1}{2}} .
$$

Here the mumerical coefficients $a_{j t n}$ satisfy the following recursion relations for $j \neq 0$

$$
\begin{aligned}
a_{j t n} & =j^{-1}\left\{(2 j+4 t+2 n-3)(6 j-4 t-2 n-1) a_{j-1, t, n-1}\right. \\
& +(2 j-4 t-2 n+5)(2 j-4 t-2 n+3) a_{j-1, t-1, n-1} \\
& +[24(j-1)(j-2 t-n)-6] a_{j-2, t, n-1}+12(1-j)(2 j-4 t-2 n+3) a_{j-2, t-1, n-1} \\
& \left.+9(2 j-5)(1-2 j) a_{j-3, t, n-1}+9(2 j-5)(2 j-1) a_{j-3, t-1, n-1}\right\}
\end{aligned}
$$

For $j=0$ we have

$$
a_{o t n}=-\sum_{j=1}^{3 n} a_{j t n}, \quad a_{000}=-2 .
$$

The complete asymptotic expansion of the incident and reflected fields is thus piven by 
(200) $u \sim e^{i k x}+\frac{1}{2} \sqrt{\frac{b_{0}}{2 s} \sin \frac{\beta}{2}} e^{i k\left(s-\frac{3}{2} \sin \frac{\beta}{2}\right)} \sum_{n=0}^{\infty} \sum_{j=0}^{3 n} \sum_{t=0}^{n}$

$$
a_{j \ln }\left(16 i b_{0} k \sin \frac{\beta}{2}\right)^{-n}\left(\frac{b_{0}}{2}\right)^{j}\left(\sin \frac{\beta}{2}\right)^{j-2 t}
$$

For the first few terms, we obtain

$$
u \sim e^{i k x}-\sqrt{\frac{b_{0}}{2 s} \sin \frac{\beta}{2}} e^{i k\left(s-\frac{3}{2} b_{0} \sin \frac{\beta}{2}\right)}\left\{1-\frac{1}{16 i b_{0} k}\left[\frac{8}{\sin ^{3} \frac{\beta}{2}}-\frac{3}{\sin \frac{\beta}{2}}\right.\right.
$$

(201)

$$
\begin{aligned}
& +\frac{b_{0}}{28}\left(\frac{1}{\sin ^{2} \frac{\beta}{2}}-3\right)+\left(\frac{b o}{2 s}\right)^{2}\left(\frac{6}{\sin \frac{\beta}{2}}-9 \sin \frac{\beta}{2}\right) \\
& \left.\left.+\left(\frac{b}{2 s}\right)^{3}\left(15 \sin ^{2} \frac{\beta}{2}-15\right)\right]+\ldots\right)
\end{aligned}
$$

These results can be used to compute the amplitude of the reflected far field. For a point in the far field (see Figure 10), $\beta \sim a, s \sim R$, and $I \sim R-2 b_{0} \sin \frac{a}{2}$. From Eq. (200) we see that the reflected field has the form

$$
u_{\text {ref }} \sim A \frac{e^{i k\left(R-2 b_{0} \sin \frac{a}{2}\right)}}{\sqrt{R}}
$$

where

(203)

$$
\begin{aligned}
& A \sim f_{00}+\frac{f_{01}}{i k}+\frac{f_{02}}{(i k)^{2}}=\ldots=-\sqrt{\frac{b_{0}}{2} \sin \frac{a}{2}} \\
& \left\{1+\frac{f_{02}}{k^{2} \sqrt{\frac{b_{0}}{2} \sin \frac{a}{2}}}+\frac{i f_{01}}{k \sqrt{\frac{b_{0}}{2} \sin \frac{a}{2}}}\right\}+\cdots \cdot
\end{aligned}
$$

Thu 8

(204)

$$
\frac{|A|}{\sqrt{\frac{b_{0}}{2} \sin \frac{a}{2}}} \sim 1+\frac{b_{0}^{2} f_{02}}{\sqrt{\frac{b_{0}}{2} \sin \frac{a}{2}\left(b_{0} k\right)^{2}}}+\frac{\left(b_{0} f_{01}\right)^{2}}{2\left(\frac{b_{0}}{2} \sin \frac{a}{2}\right)\left(b_{0} k\right)^{2}}+\cdots \cdot
$$

From (198) and (199) we finally obtain 
(205)

$$
\frac{|A|}{\sqrt{\frac{b_{0}}{2} \sin \frac{a}{2}}} \sim 1+\frac{1}{\left(16 b_{0} k\right)^{2}}\left\{\frac{3,477}{\sin ^{2} \frac{a}{2}}-\frac{7,218}{\sin ^{4} \frac{a}{2}}+\frac{3,817}{\sin ^{6} \frac{a}{2}}\right\}+\ldots .
$$

The asymptotic form of (201), for the $f a r$ field $\left(s \sim R>b_{0}\right)$ colncides with the result of I. Imai [24] obtained by expansion of the explicit solution. Since he does not give the term in $\mathrm{k}^{-2}$ his result cannot be used to compute $|A|$ given by (205) and shown in Figs. 11 and 12.

Example 20: Diffraction of a plane wave by a circular cylinder $\left(\frac{\partial u}{\partial \nu}=0\right)$

Let us reconsider the previous problem, replacing the boundary condition $u=0$ by $\frac{\partial u}{\partial v}-0$. Then instead of (193) and (194) we find

$$
v_{0}\left(s_{0}, \beta\right)=1, \quad I\left(s_{0}, \beta\right)=x=-b_{0} \sin \frac{\beta}{2}
$$

$$
\begin{aligned}
v_{n}\left(s_{0}, \beta\right)=\left(\frac{\partial x}{\partial r}\right)^{-1} \frac{\partial v_{n-1}\left(s_{0}, \beta\right)}{\partial r}= & -\frac{2 \cos \frac{\beta}{2}}{b_{0} \sin ^{2} \frac{\beta}{2}} \cdot \frac{\partial v_{n-1}\left(s_{0}, \beta\right)}{\partial \beta} \\
& -\frac{2+\cos ^{2} \frac{\beta}{2}}{2 \sin ^{2} \frac{\beta}{2}} \cdot \frac{\partial v_{n-1}\left(s_{0}, \beta\right)}{\partial s}, n \geq 1 .
\end{aligned}
$$

The phase of the reflected field is again given by (195). From (184) and (196) we again find that the $f_{j n}$ are given by (197) with $a_{j t n}$ for $j \neq 0$ satisfying (198). However for $f=0$ we have instead

(208) $a_{\text {otn }}=-\sum_{j=1}^{3 n}\left\{a_{j t n}+16(2 t+n-1) a_{j-1, t, n-1}+16(4-2 t-n-2 j) a_{j-1, t-1, n-1}\right\}$ $a_{000}=2$.

The complete asymptotic expansion of the incident and reflected field is again given by $(200)$ with the appropriate $a_{j t n}$ and for the first few terms, we obtain 


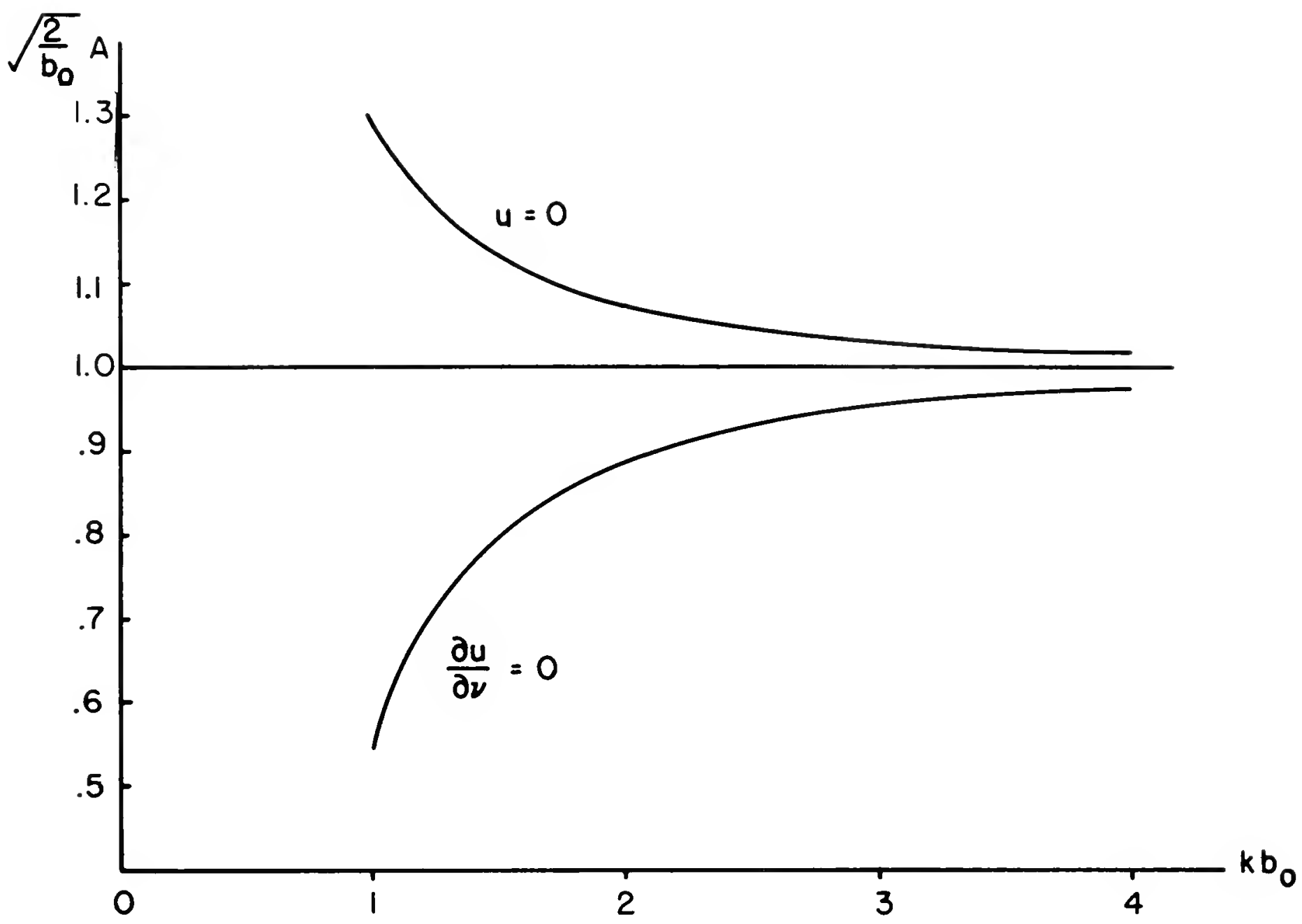

Fig. II

The back scattering amplitude $A$ divided by the geometrical optics value $\sqrt{b / 2}$ as a function of $\mathrm{kb}$. A denotes the absolute value of the amplitude of the field reflected from a circular cylinder of radius $b_{0}$ when a plane wave is incident. It is given by (210) with $a=\pi$. 
(209) $\begin{aligned} u & \sim e^{i k x}+\sqrt{\frac{b o}{2 s} \sin \frac{\beta}{2}} e^{i k\left(s-\frac{3 b o}{2} \sin \frac{\beta}{2}\right)}\left\{1+\frac{1}{16 i b k}\left[\frac{8}{\sin ^{3} \frac{\beta}{2}}+\frac{3}{\sin \frac{\beta}{2}}\right.\right. \\ & \left.\left.+\frac{b_{o}}{2 s}\left(3-\frac{1}{\sin ^{2} \frac{\beta}{2}}\right)+\left(\frac{b}{2 s}\right)^{2}\left(9 \sin \frac{\beta}{2}-\frac{6}{\sin \frac{\beta}{2}}\right)+\left(\frac{b}{2 s}\right)^{3}\left(15-15 \sin ^{2} \frac{\beta}{2}\right)\right]+\ldots\right\}\end{aligned}$

For the amplitude of the reflected far field we find instead of (205)

(210)

$$
\frac{|A|}{\sqrt{\frac{b_{0}}{2} \sin \frac{a}{2}}} \sim 1+\frac{1}{\left(16 b_{0} k\right)^{2}}\left(\frac{3,477}{\sin ^{2} \frac{a}{2}}-\frac{6,642}{\sin ^{4} \frac{a}{2}}+\frac{3,049}{\sin ^{6} \frac{a}{2}}\right)+\ldots .
$$

The asymptotic form of (209) for the far field coincides with the result of I. Imai [24] obtained by expansion of the explicit solution. As in the previous case, he does not give the term in $\mathrm{k}^{-2}$ and therefore his result cannot be used to compute $|A|$ given by (210) and shown in Figs. 11 and 12 .

\section{Axially symmetric waves}

To find the asymptotic expansion of an axially symmetric field we introduce the coordinates s, $\beta$ and $\varnothing$. Here $\emptyset$ is the ordinary rotational angle of cylindrical coordinates and $s, \beta$ are defined as above in the planes $\phi=$ const. If the caustic surface is given by the equations $z=\xi(\beta) ; r=\eta(\beta)$, then the cylindrical coordinates $z$ and $r$ are related to $s$ and $\beta$ by

$$
s \cos \beta=z-\xi ; \quad s \sin \beta=r-\eta \text {. }
$$

These relations are similar to the previous relations between $x, y$ and $s, \beta$. Therefore when the Laplacian in cylindrical coordinates is converted into $s, \beta$, $\varnothing$ coordinates, and $\emptyset$ derivatives are omitted, one obtains (179) with the additional terms

$$
\left\{\frac{\sin \beta}{\sin \beta+\eta}-\frac{\dot{\xi}}{\sin (\sin \beta+\eta)}\right\} \frac{\partial}{\partial s}+\frac{\cos \beta}{s(\sin \beta+\eta)} \cdot \frac{\partial}{\partial \beta} .
$$



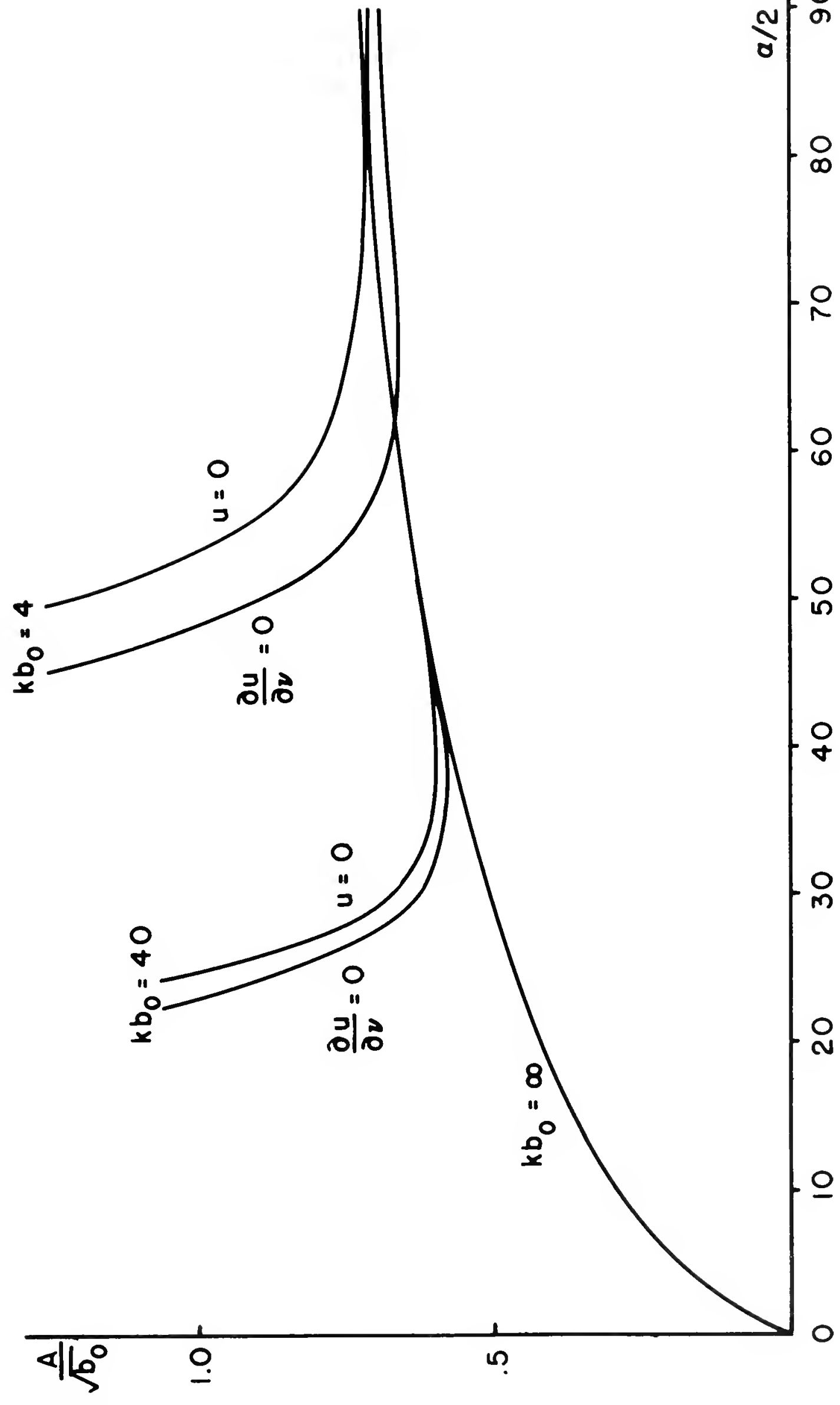

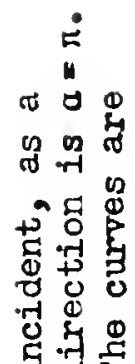

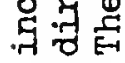

त 00

क त्र

क प

范吉

ช 긍

施

वै०

ه

욤

$\Phi$

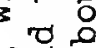

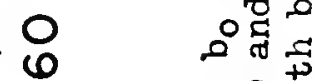

ต 0 苔

م

ป⿻ 8 क्ष

म का

4

\&

엉

दण

국결

$n$
-1
0
0
0
0

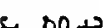

尔声古

नु

$4 \stackrel{5}{4}$

ठ

$\infty$ \&

कृष्ठ

त्व

क्ष 0

$+4$

ठํ

(口)

구

我 8

bo

㝴 สี

क 58

0 म्ने

ชั

†े

겅 응 ్ㅓㄴ 需出

돌영

+ 起罢 
Since the wavefronts of axially symmetric flelds are surfaces of revolution, the principal curvatures at any point are given by $s$ and $\csc \beta(s \sin \beta+\eta)$. Thus Eq. (6) assumes the form

$$
\begin{gathered}
v_{n}(s, \beta)=v_{n}\left(s_{0}, \beta\right)\left\{\frac{s_{0}\left(s_{0} \sin \beta+\eta\right)}{s(s \sin \beta+\eta)}\right\}^{1 / 2}-\frac{1}{2 s^{1 / 2}(s \sin \beta+\eta)^{1 / 2}} \\
\int_{s_{0}}^{s} \tau^{1 / 2}(\tau \sin \beta+\eta)^{1 / 2} \Delta v_{n-1} d \tau .
\end{gathered}
$$

Fop $\mathrm{n}=0,1,2$ we find

$$
v_{n}(s, \beta)=\{s(s \sin \beta+\eta)\}^{-1 / 2}\left\{\sum_{j=0}^{3 n} f_{j n} s^{-j}+\sum_{t=1}^{n} g_{\operatorname{tn}}(s \sin \beta+\eta)^{-t}\right\} .
$$

Here $f_{00}=v_{0}\left(s_{0}, \beta\right)\left\{s_{0}\left(s_{0} \sin \beta+\eta\right)\right\}^{1 / 2}$ and the $f_{j n}, g_{t n}$, satisfy the following recursion formulas with a, b, c given by (180) (or by (181) or (190))

$$
\text { for } \begin{aligned}
j \neq 0 ; & f_{j n}=\frac{1}{2 j}\left\{\left(j-\frac{1}{2}\right)^{2} f_{j-1, n-1}+\ddot{f}_{j-1, n-1}+c\left(j-\frac{3}{2}\right) f_{j-2, n-1}\right. \\
& +2 b(j-1) \dot{f}_{j-2, n-1} a\left(j-\frac{5}{2}\right)\left(j-\frac{1}{2}\right) f_{j-3, n-1} \\
& \left.+\sum_{\ell=j+1}^{3 n-3} \frac{(-1)^{\ell-j-1}(l-j)(\sin \beta)^{\ell-j-1} f_{\ell, n-1}}{4 \eta^{\ell-j+1}}\right\}
\end{aligned}
$$

for $j=3$, add $\quad \frac{3 a}{24} \sum_{t=1}^{n-i} \frac{e_{t, n-1}}{\eta^{t}}$

for $j=2$, add $\sum_{t=1}^{n-1}\left\{\frac{c \varepsilon_{t, n-1}+4 b \dot{q}_{t}, n-1}{8 \eta^{t}}-\frac{5 t \sin \beta g_{t, n-1}}{16 \eta^{t+1}}\right\}$ 


$$
\begin{aligned}
& \text { for } j=1 \text {, add } \sum_{t=1}^{n-1}\left\{\frac{g_{t, n-1}+4 \ddot{g}_{t, n-1}}{8 \eta^{t}} \cdot \frac{t \sin \beta\left(c g_{t, n-1}+4 b \dot{e}_{t, n-1}\right)}{4 \eta^{t+1}}\right. \\
& \left.-\frac{b t \cos \beta g_{t, n-1}}{2 \eta^{t+1}}+\frac{5 \operatorname{asin}^{2} \beta t(t+1) g_{t, n-1}}{16 \eta^{t+2}}\right\} \\
& g_{t n}=\frac{1}{2 t}\left\{\left(t-\frac{1}{2}\right)^{2} \varepsilon_{t-1, n-1}+\sum_{p=t}^{n-1}\left\{\left[\frac{5 a}{24}\left(\frac{\sin \beta}{\eta}\right)^{3}\left(_{-}^{p-t+2}\right) \frac{1}{\eta^{p-t}}-\frac{(3 p+t) \sin \beta}{4 \eta^{p-t+1}}\right.\right.\right. \\
& \left.-\frac{(p-t+1) \sin \beta}{\eta^{p-t+2}}\left(p b \cos \beta+\frac{(p-t) c \sin \beta}{4}\right)\right] g_{p, n-1}+ \\
& \left.\left[\frac{2 p \cos \beta}{\eta^{p-t+1}}-\frac{(p-t)(p-t+1) b \sin ^{2} \beta}{\eta^{p-t+2}}\right] \dot{g}_{p, n-1}+\frac{\sin \beta(p-k)}{\eta^{p-t+1}} \ddot{g}_{p, n-1}\right\} \\
& \text { for } t=1 \text {, add } \sum_{j=0}^{3 n-3} \frac{(-1)^{j}(\sin \beta)^{j-1} f_{j, n-1}}{8 \eta^{j}}
\end{aligned}
$$

For $n>2, v_{n}$ is given by $(214)$ plus additional terms in $\log s$, and $f_{j n}, g_{t n}$ still satisfy $(215)$ and (216). If the logarithmic terms cancel then (214) gives $\nabla_{n}$ completely.

Example 21: Diffraction of a plane wave by a sphere $(u=0)$

Suppose that a plane wave $e^{i k z}$ is incident from the left on a sphere given by

$$
z=-b_{0} \sin \beta / 2, \quad r=b_{0} \cos \beta / 2
$$

(see Figure 10). The caustic surface of the reflected wavefronts is given parametrically by 
(218)

$$
\begin{aligned}
& z=\xi(\beta)=-\frac{b_{0}}{2} \sin \frac{\beta}{2}\{2+\cos \beta\} \\
& r=\eta(\beta)=b_{0} \cos ^{3} \frac{\beta}{2} .
\end{aligned}
$$

Substitution of the incident plus reflected flelds into the boundary condition $u=0$ leads to the results (193) - (195). If (213) - (217)) are now used, the first few terms in the expansion of the fields are found to be

$$
\begin{aligned}
& u \sim e^{i k x}-\sqrt{\frac{b_{0}^{2} \sin \beta}{4 s\left(s \sin \beta+b_{0} \cos ^{3} \frac{\beta}{2}\right)}} e^{i k\left(s-\frac{3}{2} b_{0} \sin \frac{\beta}{2}\right)}\{1+ \\
& \frac{i}{2 b_{0} k}\left[\frac{1}{\sin ^{3} \frac{\beta}{2}}+\frac{b_{0}}{2^{5} s \sin ^{2} \frac{\beta}{2} \cos ^{2} \frac{\beta}{2}}+\frac{3}{2^{6}}\left(\frac{b_{0}}{s}\right)^{2}\left(\frac{2}{\sin \frac{\beta}{2}}-5 \sin \frac{\beta}{2}\right)\right. \\
& \left.\left.+\frac{15}{2^{7}}\left(\frac{b_{0}}{8}\right)^{3}\left(\sin ^{2} \frac{\beta}{2}-1\right)-\frac{b_{0}}{2^{4}\left(s \sin \beta+b_{0} \cos ^{3} \frac{\beta}{2}\right) \sin \frac{\beta}{2} \cos \frac{\beta}{2}}\right]+\ldots\right\} .
\end{aligned}
$$

For the far field, $s \sim R, \beta \sim a$ and $\Psi \sim R-2 b_{0} \sin \frac{\alpha}{2}$ (see Fig. 10) so that

$$
u_{r e f}=-\frac{b_{0}}{2 R} e^{i k\left(R-2 b_{0} \sin \frac{a}{2}\right)}\left\{1+\frac{i}{2 b_{0} k \sin ^{3} \frac{a}{2}}\right\}+o\left[\left(k b_{0}\right)^{-2}\right] \text {. }
$$

The result (220) agrees with the approximate solution of W. Franz and K. Depperman [25].

Example 22: Miffraction of a plane wave by a sphere $\left(\frac{\partial u}{\partial \nu}=0\right)$

If in the preceding problem, we replace the boundary condition by $\frac{\partial u}{\partial \nu}=0$, we obtain (195), (206) and (207). The first few terms in the expansion of the field are then found to be given by 
(221)

$$
\begin{aligned}
& u \sim e^{i k x}+\sqrt{\frac{b_{0}^{2} \sin \beta}{4 s\left(s \sin \beta+b_{0} \cos ^{3} \frac{\beta}{2}\right)}} e^{i k\left(s-\frac{3 b_{0}}{2} \sin \frac{\beta}{2}\right)}\{1 \\
& -\frac{1}{2 b b_{0}}\left[\frac{1+2 \sin ^{2} \frac{\beta}{2}}{\sin ^{3} \frac{\beta}{2}}-\frac{b_{0}}{2^{5} \sin \sin ^{2} \frac{\beta}{2} \cos ^{2} \frac{\beta}{2}}-\frac{3}{2^{6}}\left(\frac{b_{0}}{8}\right)^{2}\left(\frac{2}{\sin \frac{\beta}{2}}-5 \sin \frac{\beta}{2}\right)\right. \\
& \left.\left.-\frac{15}{2^{7}}\left(\frac{b_{0}}{8}\right)^{3}\left(\sin ^{2} \frac{\beta}{2}-1\right)-\frac{b_{0}}{2^{4}\left(s \sin \beta+b_{0} \cos ^{3} \frac{\beta}{2}\right) \sin \frac{\beta}{2} \cos \frac{\beta}{2}}\right]+\ldots\right\} .
\end{aligned}
$$

For the far field, we find

(222) $u_{r e f}=\frac{b_{0}}{2 R} e^{i k\left(R-2 b_{0} \sin \frac{a}{2}\right)}\left\{1-1 \frac{1+2 \sin ^{2} \frac{a}{2}}{2 b_{0} k \sin ^{3} \frac{a}{2}}\right\}+0\left[\left(b_{0} k\right)^{-2}\right]$.

The result (222) agrees with the approximate solution of W. Franz and K. Tepperman [25].

10. The impedance boundary condition

We will now consider reflection from a surface on which an impedance (or mixed) boundary condition is to be satisfied. The impedance boundary condition is

$$
\frac{\partial u}{\partial \nu}=i k z u \text {. }
$$

In (223) $\mathrm{Z}$ is called the impedance of the boundary and wo will assume that it is independent of $k$.

Let the incident and reflected fields have the expansions 
(224)

$$
u_{i n c} \sim e^{i k \Psi_{0}(x, y, z)} \sum_{n=0}^{\infty} \frac{w_{n}(x, y, z)}{(i k)^{n}}
$$

(225)

$$
u_{r e f} \sim e^{i k^{\bar{\psi}}(x, y, z)} \sum_{n=0}^{\infty} \frac{\nabla_{n}(x, y, z)}{(i k)^{n}} .
$$

Then, applying the impedance boundary condition (223) to the total field we find that the following relations hold on the boundary

$$
\begin{aligned}
& \mathbf{I}=\mathbf{I}_{0}, \quad \frac{\partial \mathbf{Y}}{\partial \nu}=-\frac{\partial \mathbf{Y}_{0}}{\partial \nu} \\
& v_{0}=\left(-z+\frac{\partial Y_{0}}{\partial v}\right)\left(z+\frac{\partial Y_{0}}{\partial v}\right)^{-I} w_{0} \\
& v_{n}=\left(\frac{\partial v_{0}}{\partial \nu}+z\right)^{-1}\left\{\left(\frac{\partial \bar{r}_{0}}{\partial \nu}-z\right) w_{n}+\frac{\partial w_{n-1}}{\partial \nu}+\frac{\partial v_{n-1}}{\partial \nu}\right\}, \quad n \geq 1 .
\end{aligned}
$$

The limits $Z \rightarrow \infty$ and $Z \rightarrow 0$ correspond, respectively, to the boundary conditions $u=0$ and $\frac{\partial u}{\partial v}=0$.

Example 23: Diffraction of a plane wave by a parabolic cylinder $\left(\frac{\partial u}{\partial v}=i k z u\right)$ A plane wave $e^{-i k x}$ is incident from the right (see Figure 1 ) on the parabolic cylinder with $E_{1} \cdot(16)$. From Eq8. (226) - (228), we find that on the parabolic cylinder

$$
\begin{gathered}
Y=-x=-r_{0}(\theta) \cos \theta \\
v_{0}=\left(z+\cos \frac{\theta}{2}\right)\left(-Z+\cos \frac{\theta}{2}\right)^{-1} \\
v_{n}=-\frac{1}{\cos \frac{\theta}{2}-z} \cdot \frac{\partial v_{n-1}}{\partial v}=-\frac{1}{\cos \frac{\theta}{2}-z}\left\{\cos \frac{\theta}{2} \frac{\partial v_{n-1}}{\partial r}-\frac{\sin \frac{\theta}{2} \cos \frac{\theta}{2}}{p} \frac{\partial v_{n-1}}{\partial \theta}\right\} \cdot
\end{gathered}
$$

If these initial values are inverted into the recursion formulas ( 9 ) one finds and verifies inductively that for $n \geq 1$ 
(232)

$$
f_{j n}=p^{-n+j+\frac{1}{2}}\left(\cos \frac{\theta}{2}-2\right)^{-(2 n+1)} \sum_{t=0}^{4 n} a_{j t n}\left(\cos \frac{\theta}{2}\right)^{t+2 j-1} .
$$

For $j \neq 0$ and $n \geq 2$ the constants $a_{j t n}$ satisfy

$$
\begin{aligned}
a_{j t n} & =j^{-1}\left\{(t-2 n-3)(4 j+2 n-t+1) a_{j-1, t-4, n-1}\right. \\
& +z[(t-3)(t-4 j-1)+(t-4 j)(t-2)-2 n(2 t-4 j-3)] a_{j-1, t-3, n-1} \\
& +z(t-2 j)(4 j+4 n-2 t) a_{j-1, t-1, n-1} \\
& +\left[z^{2}(4 j-t)(t-2)+(2 n+2 j+1-t)(2 n+2 j-t)\right] a_{j-1, t-2, n-1} \\
& \left.+z^{2}(t-2 j)(t-2 j+1) a_{j-1, t, n-1}\right\}
\end{aligned}
$$

For $f=0$ we have instead

$$
\begin{aligned}
a_{o t n}=-\sum_{j=1}^{n} & \left\{a_{j t n}+\frac{1}{2}\left[(3+2 n-t) a_{j-1, t-4, n-1}+2(t-3) a_{j-1, t-3, n-1}\right.\right. \\
& \left.\left.(t-2 j-2 n) a_{j-1, t-2, n-1}+2(2 j-t) a_{j-1, t-1, n-1}\right]\right\} .
\end{aligned}
$$

The complete asymptotic expansion of the field is thus given by

$$
\begin{aligned}
u \sim \theta^{-i k x}+\sqrt{\frac{p}{r}} \frac{e^{i k(r-2 p)}}{\cos \frac{\theta}{2}\left(\cos \frac{\theta}{2}-2\right)} \sum_{n=0}^{\infty} \sum_{j=1}^{n} \sum_{t=0}^{4 n} \\
\left\{a_{j t n}\left[i k p\left(\cos \frac{\theta}{2}-z\right)^{2}\right]^{-n}\left(\frac{p}{r}\right)^{j}\left(\cos \frac{\theta}{2}\right)^{t-2 j}\right\} .
\end{aligned}
$$

The first few terms are 
(236) $u \sim e^{-i k x}+\sqrt{\frac{p}{r}} \frac{e^{i k(r-2 p)}}{\cos \frac{\theta}{2}\left(\cos \frac{\theta}{2}-z\right)}\left\{z+\cos \frac{\theta}{2}+\frac{1}{41 p k\left(\cos \frac{\theta}{2}-2\right)^{2}}\left[-2 \cos ^{4} \frac{\theta}{2}\right.\right.$

$+\left(1-z^{2}\right) \cos ^{3} \frac{\theta}{2}+2 \cos ^{2} \frac{\theta}{2}+z^{2} \cos \frac{\theta}{2}-z^{3}+\frac{p}{r}\left(-3 z \cos ^{2} \frac{\theta}{2}+\left(z^{2}+1\right) \cos \frac{\theta}{2}\right.$ $\left.\left.\left.+3 z-\frac{3 z^{2}}{\cos \frac{\theta}{2}}+\frac{z^{3}}{\cos ^{2} \frac{\theta}{2}}\right)\right]+\ldots\right\}$

For the far field we find that

(237)

$$
\begin{aligned}
& u_{r e f}=\sqrt{\frac{p}{r}} \frac{e^{i k(r-2 p)}}{\cos \frac{\theta}{2}\left(\cos \frac{\theta}{2}-z\right)}\left\{z+\cos \frac{\theta}{2}+\frac{1}{41 p k\left(\cos \frac{\theta}{2}-z\right)^{2}}\left[-z \cos ^{4} \frac{\theta}{2}\right.\right. \\
&\left.+\left(1-z^{2}\right) \cos ^{3} \frac{\theta}{2}+2 \cos ^{2} \frac{\theta}{2}+z^{2} \cos \frac{\theta}{2}-z^{3}\right]+\frac{1}{\left[8 p k\left(\cos \frac{\theta}{2}-z\right)^{2}\right]^{2}}\left[15 z \cos ^{8} \frac{\theta}{2}\right. \\
&-43 z^{2} \cos ^{7} \frac{\theta}{2}+\left(44 z-17 z^{3}\right) \cos ^{6} \frac{\theta}{2}+\left(9 z^{4}+20 z^{2}-16\right) \cos ^{5} \frac{\theta}{2} \\
&\left.\left.+\left(40 z-8 z^{3}\right) \cos ^{4} \frac{\theta}{2}+\left(32 z^{2}-8 z^{4}\right) \cos ^{3} \frac{\theta}{2}+16 z^{3} \cos ^{2} \frac{\theta}{2}-24 z^{4} \cos \frac{\theta}{2}+8 z^{5}\right]\right\} \\
&+0[(k p)-3]
\end{aligned}
$$

The amplitude of the far field is

(238) $\frac{|A|}{\sqrt{p} \sec \frac{\theta}{2}} \cdot \frac{\left(\cos \frac{\theta}{2}-Z\right)}{\left(\cos \frac{\theta}{2}+Z\right)} \sim 1+\frac{1}{\left[8 \mathrm{pk}\left(\cos \frac{\theta}{2}-Z\right)\right]^{2}\left(\cos ^{2} \frac{\theta}{2}-Z^{2}\right)}\left\{15 Z \cos ^{9} \frac{\theta}{2}\right.$

$$
\left.\begin{array}{l}
-26 z^{2} \cos ^{8} \frac{\theta}{2}+\left(40 z-40 z^{3}\right) \cos ^{7} \frac{\theta}{2}+\left(56 z^{2}-14\right) \cos ^{6} \frac{\theta}{2} \\
+\left(9 z^{5}+4 z^{3}+28 z\right) \cos ^{5} \frac{\theta}{2}+\left(78 z^{2}-16 z^{4}\right) \cos ^{4} \frac{\theta}{2}+\left(48 z^{3}-4 z^{5}\right) \cos ^{3} \frac{\theta}{2} \\
-10 z^{4} \cos ^{2} \frac{\theta}{z}-20 z^{5} \cos \frac{\theta}{2}+10 z^{6}
\end{array}\right\}
$$


- 77 -

Example 241 Diffraction of a plane wave by a circular cylinder $\left(\frac{\partial u}{\partial v}=i k Z u\right)$

If a plane wave $e^{i k x}$ is incident on the circular cylinder given by Eq. (192), we find from (226)-(228) using the coordinates $(s, \beta)$ that

$$
\begin{aligned}
Y\left(s_{0}, \beta\right) & =-b_{0} \sin \frac{\beta}{2} \\
v_{0}\left(s_{0}, \beta\right) & =\left(Z+\sin \frac{\beta}{2}\right)\left(\sin \frac{\beta}{2}-z\right)^{-1} \\
v_{n}\left(s_{0}, \beta\right) & =\frac{1}{2-\sin \frac{\beta}{2}} \cdot \frac{\partial v_{n-1}}{\partial r} \\
& =\frac{2 \cos \frac{\beta}{2}}{\sin \frac{\beta}{2}\left(Z-\sin \frac{\beta}{2}\right)} \cdot \frac{\partial v_{n-1}}{\partial \beta}+\frac{2+\cos ^{2} \frac{\beta}{2}}{2 \sin \frac{\beta}{2}\left(Z-\sin \frac{\beta}{2}\right)} \cdot \frac{\partial v_{n-1}}{\partial s}, n \geq 1
\end{aligned}
$$

The first few terms of the asymptotic expansion of the field are found to be (242)

$$
\begin{aligned}
& u \sim e^{i k x}+\sqrt{\frac{b_{0}}{2 s} \sin \frac{\beta}{2}} \frac{e^{i k\left(s-\frac{3}{2} b_{0} \sin \frac{\beta}{2}\right)}}{\sin \frac{\beta}{2}-2}\left\{z+\sin \frac{\beta}{2}-\frac{i}{b_{0} k}\left(\frac{1}{2^{4}\left(\sin \frac{\beta}{2}-z\right)^{2}}\right.\right. \\
& {\left[3 \sin ^{2} \frac{\beta}{2}-11 z \sin \frac{\beta}{2}+8-192^{2}\right.} \\
& \left.+\frac{8 z+3 z^{3}}{\sin \frac{\beta}{2}}+\frac{8 z^{2}}{\sin ^{2} \frac{\beta}{2}}-\frac{8 z^{3}}{\sin ^{3} \frac{\beta}{2}}\right]+\frac{b_{0}}{\left.2^{5} \sin \frac{\beta}{2}-2\right)^{2}}\left[3 \sin ^{3} \frac{\beta}{2}\right. \\
& \left.-3 z \sin ^{2} \frac{\beta}{2}-\left(1+11 z^{2}\right) \sin \frac{\beta}{2}+9 z+3 z^{3}+\frac{9 z^{2}}{\sin \frac{\beta}{2}}-\frac{z^{3}}{\sin ^{2} \frac{\beta}{2}}\right] \\
& +\frac{3 b_{0}^{2}}{2^{6} \sin ^{2}\left(\sin \frac{\beta}{2}-z\right)}\left[3 \sin ^{3} \frac{\beta}{2}-\left(3 z^{2}+2\right) \sin \frac{\beta}{2}+8 z+\frac{2 z^{2}}{\sin \frac{\beta}{2}}-\frac{8 z}{\sin ^{2} \frac{\beta}{2}}\right] \\
& \left.\left.+\frac{15 b_{0}^{3}}{2^{7} s^{3}}\left[-\sin ^{3} \frac{\beta}{2}-2 \sin ^{c} \frac{\beta}{2}+\sin \frac{\beta}{2}+2\right]\right)\right\}+\cdots
\end{aligned}
$$




\section{References}

[1] Luneburg, R.K., a. The Mathematical Theory of Optics, Brown Uriv., 1:44 b. Propagation of Electromagnetic Waves, New Ycrk Univ., 1948.

[2] Kline, M., An Asymptotic Solution of Maxwell's Fquaticns, Comm. on Pure and Applied Math., Vol. IV, No. 2-3, Aug. 1951, pp. 225-263 and Asymptotic Solution of Iinear Hyperbolic Partial Differential Equations, Journ. of Rational Mech. and Analysis, Vol. 3, No. 3, Mey, 1954.

[3] Frledlander, F.G., Geometrical Optics and Maxwell 's Equations, Proc. Cambridge Phil. Soc. Vol. 43, Pt. 2, 1946, pp. 234-286.

[4] Bremmer, H., The Jumps of Discontinuous Solutions of the Wave Equation, Comm. on Pure and Applied Math., Vol. IV, No. 4, Nov. 1951 pp. 419-427.

[5] Copson, E.T., The Transport of Discontinuities in an Electromagnetic Field, Comm. on Pure and Applied Math., Vol. IV, No. 4, Nov. 1951, pp. $427-435$.

[5] Keller, J.B., The Geometrical Theory of Diffraction, Proceedings of the Symposium on Microwave Optics, McGill Untv., June 1953.

[7] Keller, J.J., Geometrical Acoustics I. The Theory of Weak Shocks, Journ. Appl. Physics, Vol. 25, No. 8, pp. 938-947, Aug. 1954.

[B] Birkhoff, G.D., Some Remarks Concerning Schroedinger's Wave Equation, Froc. Nat'1. Acad. of Sciences, Vol. 19, 1933 pp. 339-34l and in Collected Math. Papers, Amer. Math. Soc., 1950, Vol. II, pp. 813818. Also quantum Mechanics and Asymptotic Series, Amer. Math. Soc. Bulletin, Vol. 39, 1933, pp. 681-700 and in Collected Math. Papers, VoI. II, NP. 837-856.

[9] Brillouin, L., Remarques sur la Mecanique Ondulatoire, J。 Phys. Radium 7, pp. 353-368 (1936) 3 Las Mecanique Ondulatoire; une methode generale de resolution por approximations successives Comptes Rendus 183, $24,1926$.

[10] Dirac, P.A.M., Tho Frinciples of Quantum Mechenics, Oxford, 1947, Third Edition, pp. 121-123.

[1]] Keller, J.B., Derivation of the Bohr-Sommerfeld Quantum Conditions From an Asymptotic Solution of the Schroedinger Equation. Research Report No. CX-10, July, 1953, Institute of Mathematical Sciences, New York University, Division of Electromagnetic Research, N.I.

[12] Lowell, S.C., The Propagation of Waves in Shallow Water, Comm. on Fure and Applied Math. Vol. 2, No. 213, Fp. 275-291, 1949. 
[13] Schensted, C., The Electromagnetic Transport Equation and the LuneburgKline Method of Solution, Willow kun Research Center, Eng. Research Inst., Uni.v. of Michigan, Report 15-25-(504)-3.

[14] Friedrichs, K.O. and Keller, J.B., Geometrical Acoustics II: Diffraction, Reflection and Refraction of a Weak Spherical or Cylindrical Shock at a Plane Interface, to be published in Jour. Appl. Phys.

[15] Friedlander, F.G. and Keller, J.B., Asymptotic Expansions of Solutions of $\left(\nabla^{2}+k^{2}\right) u=0$, Research Report No. EM-67, Sept. 1954, Institute of Mathematical Sciences, New York University, Division of Electromagnetic Research, N.Y.

[16] Kay, I. and Keller, J.B., Asymptotic Evaluation of the Field at a Caustic, Jour. Appl. Phys., Vol. 25, No. 7, pp. 876-883, July, 1954.

[17] Gerjuoy, E., Total Reflection of Waves From a Point Source, Comm. on Pure and Applied Math., Vol. VI, No. 1, pp. 73-91, 1953.

[18] Lamb, H., On Sommerfeld's Diffraction Problem and On Reflection by a Parabolic Mirror, Proc. London Math. Soc., 1906, pp. 190-203.

[19] Friedlander, F.G., On the Reflexion of a Spherical Sound Pulse by a Parabolic Mirror, Proc. Comb. Phil. Soc., Vol. 38, Pt. 4, pp. 383393.

[20] Erdelyi, A., et. al., Higher Transcendental Functions, McGraw-Hill, New York, 1953.

[21] Wentzel, G., Eine Verallgemeinerung der Quantenbedingungen fur die Zwecke der Wellenmechanik, Zeits, f. Physik, Vol. 38, p. 518, 1926.

[22] Keller, J.B.- and Keller, H.B., Determination of Reflected and Transmitted Fields by Geometrical Optics, Jour. Optical Soc. Am., Vol 40, No. 1, pp. 48-52, Jan. 1950.

[23] Keller, J.B. and Preiser, S., Determination of Reflected and Transmitted Fields by Geometrical Optics II, Inst. Math. Sciences, New York University, Division of Electromagnetic Research, Report No. EM-20, April, 1950.

[24] Imai, I., Die Beugung elektromagnetischer Wellen an einem Kreiszylinder, Zeit. fur Physik, Bd. 137, pp. 31-48 (1954).

[25] Depperman, K. and Franz, W., Theorie der Beugung an der Kugel unter Berucksichtigung der Kriechwelle, Ann. der Physik, Ser. 6, Vol. 14 pp. 253-264, 1954 . 




\section{Date Due}

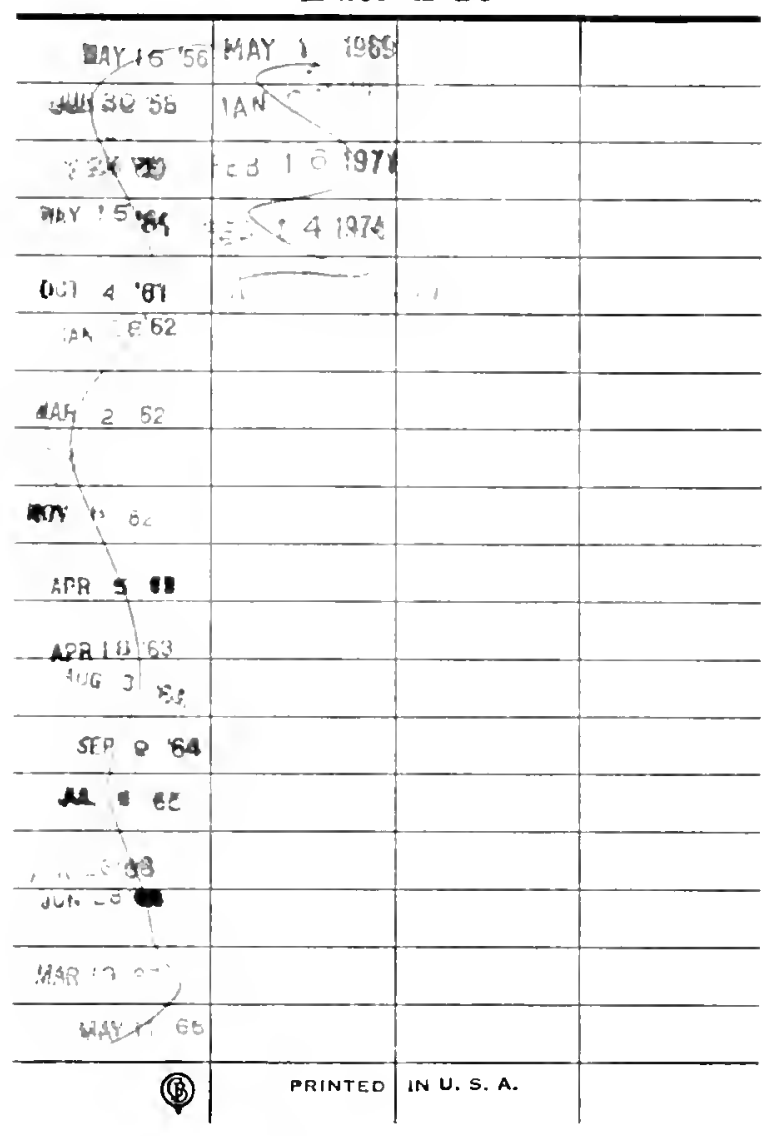




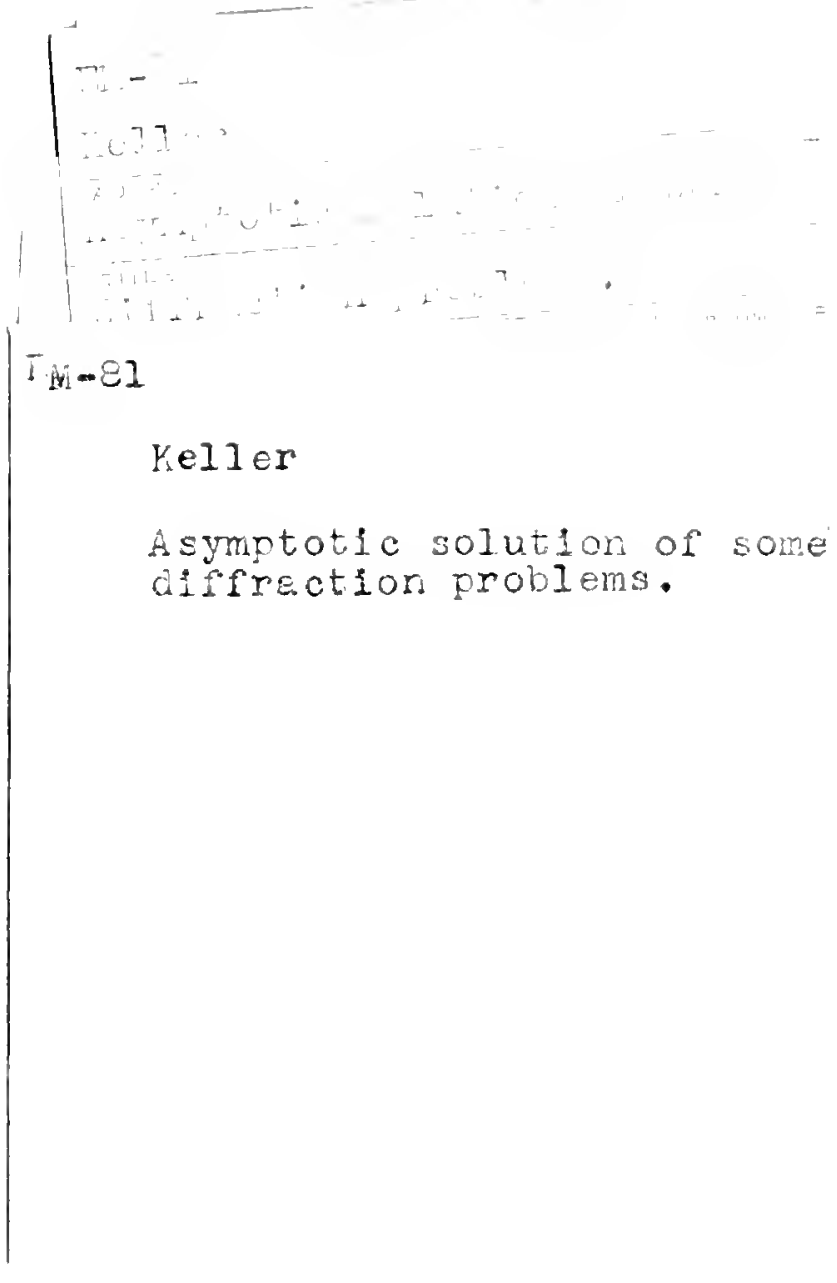


